\title{
The Preparation of 4-(Nitromethyl)furan Derivatives and Their Application in the Syntheses of Bis(furan-2-yl)oximes
}

\author{
Yinbo Wan ${ }^{a, b}$, Yang Zhu ${ }^{a, b}$, Haiyun Peng ${ }^{a, b}$, Guisheng Deng ${ }^{a, b, *}$
}

${ }^{a}$ Key Laboratory of Chemical Biology and Traditional Chinese Medicine Research (Ministry of Education of China), Hunan Normal University, Changsha 410081, China

${ }^{b}$ Key Laboratory of the Assembly and Application of Organic Functional Molecules of Hunan Province, Hunan Normal University, Changsha 410081, China.

*E-mail: gsdeng@hunnu.edu.cn

\section{Table of Contents}

1. Scheme S1. Approaches to Synthesis of oxime 9.................S2

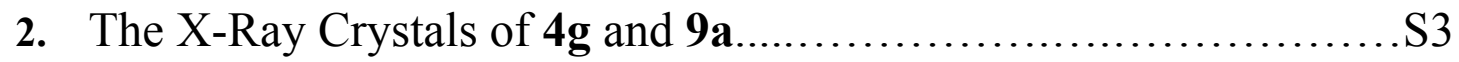

3. ${ }^{1} \mathrm{H}$ NMR and ${ }^{13} \mathrm{C}\left\{{ }^{1} \mathrm{H}\right\}$ NMR Spectra for all new compounds...S5-S52 


\section{Scheme S1. Approaches to Synthesis of oxime 9}

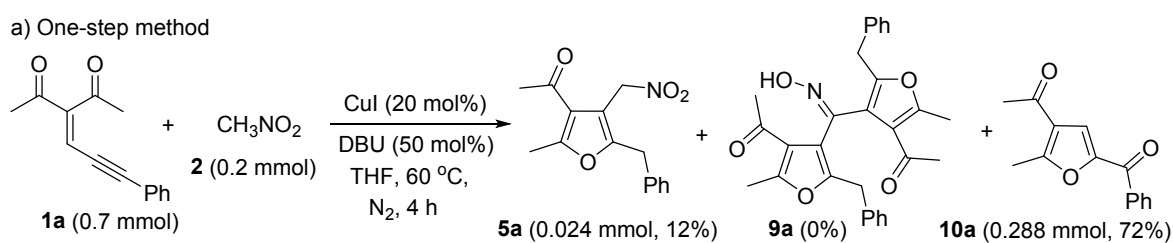

b) One-pot method

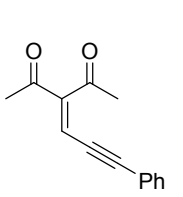

1) $\mathrm{Cu}\left(\mathrm{CH}_{3} \mathrm{CN}\right)_{4} \mathrm{PF}_{6}$ (10 mol\%)

DBU (50 mol\%) $\mathrm{CH}_{3} \mathrm{NO}_{2}: \mathrm{DCE}=1: 4$ $25^{\circ} \mathrm{C}, 2 \mathrm{~h}, \mathrm{~N}_{2}$

2) $\mathrm{Cul}(10 \mathrm{~mol} \%)$ DBU (20 mol\%)

1a (1.2 mmol) THF, $60^{\circ} \mathrm{C}, 2.5 \mathrm{~h}, \mathrm{~N}_{2}$

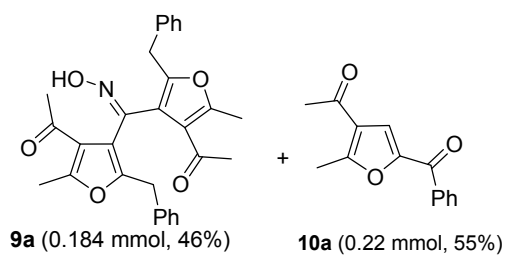

c) Co-catalytic method

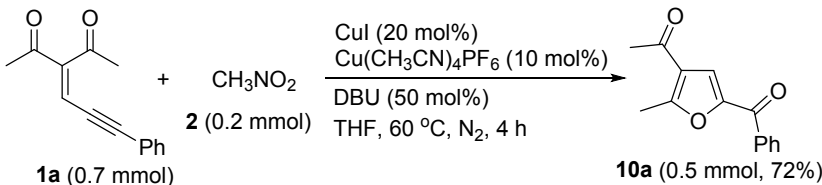

d) Method starting from substrates $\mathbf{1}$ and $\mathbf{5}$

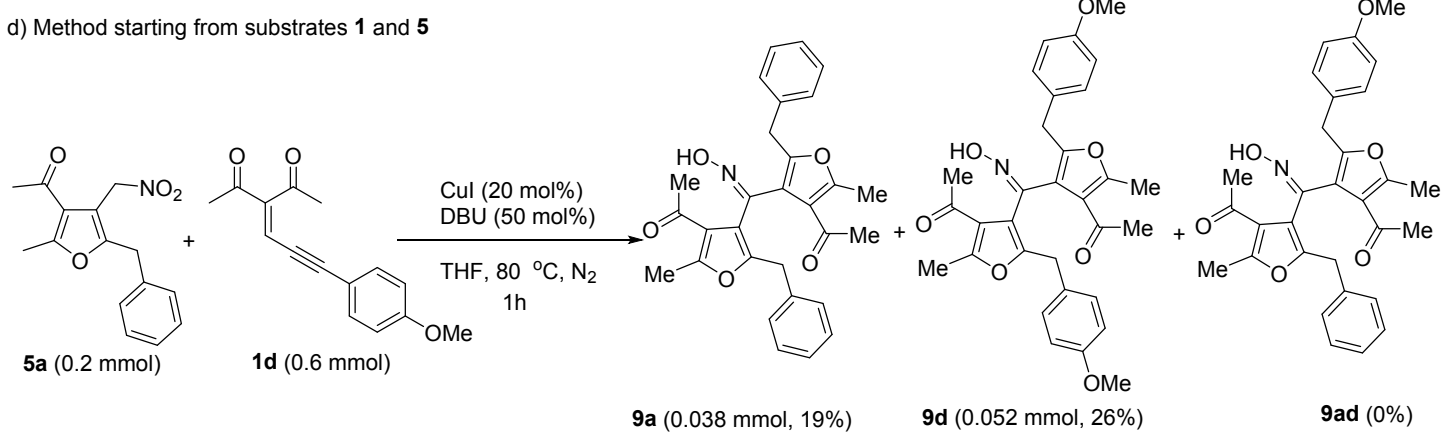

On the base of catalytic activity of $\mathrm{Cu}(\mathrm{I})$ for the construction of compounds 5 and $\mathbf{9}$, three methods for synthesis of oxime 9 from substrates 1 and $\mathbf{2}$ have been examined in CuI/DBU/THF $/ 60^{\circ} \mathrm{C}$ system. The oxidation of (2-furyl)copper carbene generated in situ from 1a by nitromethane should occur, providing predominant 10a without the desired product 9a (Scheme S1, a). In a one-pot procedure, the mixture of $1 \mathrm{a}, \mathrm{Cu}\left(\mathrm{CH}_{3} \mathrm{CN}\right)_{4} \mathrm{PF}_{6}$, DBU and nitromethane 2 in DCE was stirred at $25^{\circ} \mathrm{C}$ under $\mathrm{N}_{2}$ for two hours; $\mathrm{DCE}$ was removed in vacuum, providing a residue; the residue and $\mathrm{CuI}$ were dissolved in THF, and then the mixture was stirred at $60^{\circ} \mathrm{C}$ under $\mathrm{N}_{2}$ for another 2.5 hours, resulting in the formation of the desired product 9a in lower yield (Scheme S1, b). In $\mathrm{Cu}\left(\mathrm{CH}_{3} \mathrm{CN}\right)_{4} \mathrm{PF}_{6} / \mathrm{CuI} / \mathrm{DBU} / \mathrm{THF} / 60{ }^{\circ} \mathrm{C} / \mathrm{N}_{2}$ system, the utilization of $1 \mathrm{a}$ did not afford the desired product 9a as well except for compound 10a (Scheme S1, c). Obviously, all these attempts for convenient synthesis of $\mathbf{9 a}$ were unsuccessful. The reaction of compound 1d with $\mathbf{5 a}$ only afforded a mixture of two symmetric products $9 \mathbf{a}$ and $9 \mathbf{d}$ without the desired asymmetric product 9ad, The ratio of product 9a to product 9d was about 3: 4 based on ${ }^{1} \mathrm{H}$ NMR analysis because it was difficult to separated products 9a and 9d by column chromatography on silica gel (Scheme S1, d). 


\section{The X-Ray Crystal of $\mathbf{4 g}$ and 9a}

Important Crystal Data for 4g. Crystallographic data have been deposited with the Cambridge Crystallographic Data Centre; deposition No. CCDC-2017105.

\begin{tabular}{|c|c|c|}
\hline Identification code & \multicolumn{2}{|l|}{ CCDC (2017105) } \\
\hline Empirical formula & \multicolumn{2}{|l|}{ C15 H14 F N O4 } \\
\hline Formula weight & \multicolumn{2}{|l|}{291.27} \\
\hline Temperature & \multicolumn{2}{|l|}{$293 \mathrm{~K}$} \\
\hline Wavelength & \multicolumn{2}{|l|}{$1.54184 \AA$} \\
\hline Crystal system & \multicolumn{2}{|l|}{ Monoclinic } \\
\hline Space group & \multicolumn{2}{|l|}{ P $121 / \mathrm{c} 1$} \\
\hline \multirow[t]{3}{*}{ Unit cell dimensions } & $\mathrm{a}=10.9391(3) \AA$ & $\alpha=90^{\circ}$. \\
\hline & $\mathrm{b}=11.6158(3) \AA$ & $\beta=95.9518(19)^{\circ}$. \\
\hline & $\mathrm{c}=10.8989(2) \AA$ & $\gamma=90^{\circ}$ \\
\hline Volume & \multicolumn{2}{|l|}{$1377.41(5) \AA^{3}$} \\
\hline Z & \multicolumn{2}{|l|}{4} \\
\hline Density (calculated) & \multicolumn{2}{|l|}{$1.405 \mathrm{Mg} / \mathrm{m}^{3}$} \\
\hline Absorption coefficient & \multicolumn{2}{|l|}{$0.942 \mathrm{~mm}^{-1}$} \\
\hline $\mathrm{F}(000)$ & \multicolumn{2}{|l|}{608} \\
\hline Crystal size & \multicolumn{2}{|l|}{$0.3 \times 0.3 \times 0.1 \mathrm{~mm}^{3}$} \\
\hline Theta range for data collection & \multicolumn{2}{|l|}{4.063 to $66.587^{\circ}$. } \\
\hline Index ranges & \multicolumn{2}{|c|}{$-13<=\mathrm{h}<=13,-13<=\mathrm{k}<=13,-12<=1<=12$} \\
\hline Reflections collected & \multicolumn{2}{|l|}{16784} \\
\hline Independent reflections & \multicolumn{2}{|c|}{$2424[\mathrm{R}(\mathrm{int})=0.0322]$} \\
\hline Completeness to theta $=66.587^{\circ}$ & \multicolumn{2}{|l|}{$100.0 \%$} \\
\hline Absorption correction & \multicolumn{2}{|c|}{ Semi-empirical from equivalents } \\
\hline Max. and min. transmission & \multicolumn{2}{|c|}{1.00000 and 0.36764} \\
\hline Refinement method & \multicolumn{2}{|c|}{ Full-matrix least-squares on $\mathrm{F}^{2}$} \\
\hline Data / restraints / parameters & \multicolumn{2}{|l|}{$2424 / 0 / 192$} \\
\hline Goodness-of-fit on $\mathrm{F}^{2}$ & \multicolumn{2}{|l|}{1.067} \\
\hline Final $\mathrm{R}$ indices [I>2sigma(I)] & \multicolumn{2}{|c|}{$\mathrm{R} 1=0.0445, \mathrm{wR} 2=0.1277$} \\
\hline $\mathrm{R}$ indices (all data) & \multicolumn{2}{|c|}{$\mathrm{R} 1=0.0491, \mathrm{wR} 2=0.1315$} \\
\hline Extinction coefficient & \multicolumn{2}{|l|}{$\mathrm{n} / \mathrm{a}$} \\
\hline Largest diff. peak and hole & \multicolumn{2}{|c|}{0.305 and $-0.234 \mathrm{e} . \AA^{-3}$} \\
\hline
\end{tabular}

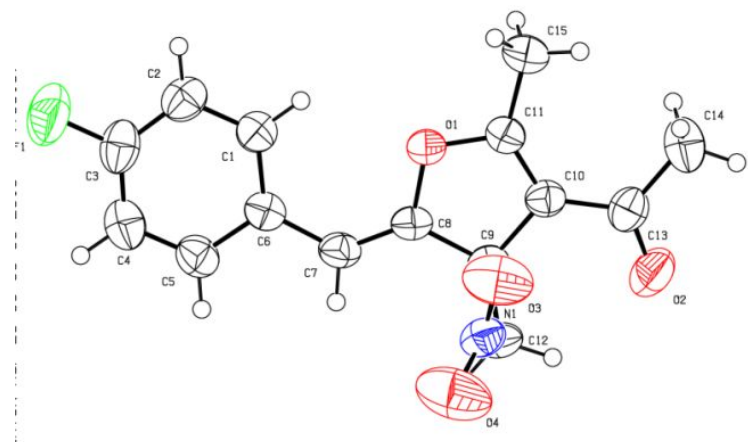

Figure S1 X-ray crystal structure of $\mathbf{4 g}$

Note: the sample was prepared from Dichloromethane/n-hexane $=1: 4$ system via slow evaporation; crystal structure of $\mathbf{4 g}$ is at $50 \%$ probability levels for the thermal ellipsoid plot. 
Important Crystal Data for 9a. Crystallographic data have been deposited with the Cambridge Crystallographic Data Centre; deposition NO. CCDC-1994626.

\begin{tabular}{|c|c|c|}
\hline Identification code & \multicolumn{2}{|l|}{ CCDC (1994626) } \\
\hline Empirical formula & \multicolumn{2}{|l|}{ C29 H27 N O5 } \\
\hline Formula weight & \multicolumn{2}{|l|}{469.51} \\
\hline Temperature & \multicolumn{2}{|l|}{ 293(2) K } \\
\hline Wavelength & \multicolumn{2}{|l|}{$1.54184 \AA$} \\
\hline Crystal system & \multicolumn{2}{|l|}{ Orthorhombic } \\
\hline Space group & \multicolumn{2}{|l|}{ Pn 21 a } \\
\hline \multirow[t]{3}{*}{ Unit cell dimensions } & $\mathrm{a}=11.6842(2) \AA$ & $\alpha=90^{\circ}$. \\
\hline & $\mathrm{b}=12.03136(19) \AA$ & $\beta=90^{\circ}$. \\
\hline & $\mathrm{c}=17.8762(2) \AA$ & $\gamma=90^{\circ}$ \\
\hline Volume & \multicolumn{2}{|l|}{$2512.97(7) \AA^{3}$} \\
\hline Z & \multicolumn{2}{|l|}{4} \\
\hline Density (calculated) & \multicolumn{2}{|l|}{$1.241 \mathrm{Mg} / \mathrm{m}^{3}$} \\
\hline Absorption coefficient & \multicolumn{2}{|l|}{$0.687 \mathrm{~mm}^{-1}$} \\
\hline $\mathrm{F}(000)$ & \multicolumn{2}{|l|}{992} \\
\hline Crystal size & \multicolumn{2}{|l|}{$0.3 \times 0.3 \times 0.2 \mathrm{~mm}^{3}$} \\
\hline Theta range for data collection & \multicolumn{2}{|l|}{4.430 to $66.580^{\circ}$. } \\
\hline Index ranges & \multicolumn{2}{|c|}{$-12<=\mathrm{h}<=13,-14<=\mathrm{k}<=14,-21<=\mathrm{l}<=21$} \\
\hline Reflections collected & \multicolumn{2}{|c|}{29559} \\
\hline Independent reflections & \multicolumn{2}{|l|}{$4420[\mathrm{R}(\mathrm{int})=0.0650]$} \\
\hline Completeness to theta $=66.580^{\circ}$ & \multicolumn{2}{|l|}{$100.0 \%$} \\
\hline Absorption correction & \multicolumn{2}{|c|}{ Semi-empirical from equivalents } \\
\hline Max. and min. transmission & \multicolumn{2}{|c|}{1.00000 and 0.47101} \\
\hline Refinement method & \multicolumn{2}{|c|}{ Full-matrix least-squares on $\mathrm{F}^{2}$} \\
\hline Data / restraints / parameters & \multicolumn{2}{|l|}{$4420 / 1 / 321$} \\
\hline Goodness-of-fit on $\mathrm{F}^{2}$ & \multicolumn{2}{|l|}{1.063} \\
\hline Final $R$ indices $[I>2 \operatorname{sigma}(I)]$ & \multicolumn{2}{|c|}{$\mathrm{R} 1=0.0539, \mathrm{wR} 2=0.1490$} \\
\hline $\mathrm{R}$ indices (all data) & \multicolumn{2}{|c|}{$\mathrm{R} 1=0.0553, \mathrm{wR} 2=0.1514$} \\
\hline Absolute structure parameter & \multicolumn{2}{|l|}{$0.11(12)$} \\
\hline Extinction coefficient & \multicolumn{2}{|l|}{$\mathrm{n} / \mathrm{a}$} \\
\hline Largest diff. peak and hole & \multicolumn{2}{|l|}{0.717 and -0.262 e. $\AA^{-3}$} \\
\hline
\end{tabular}

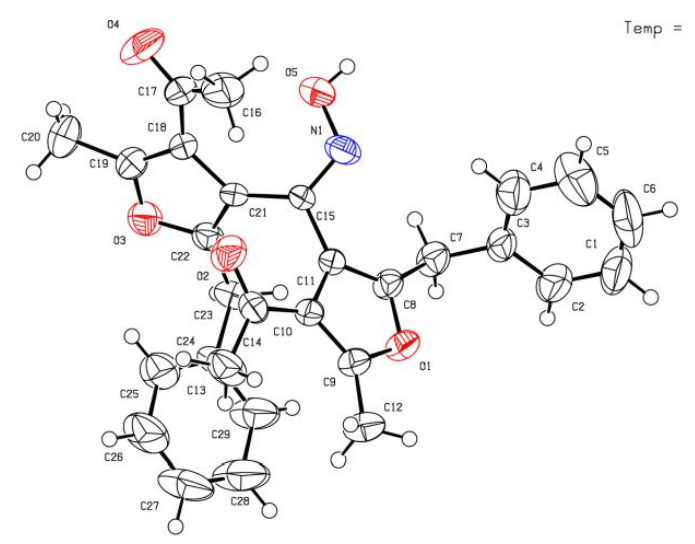

Figure S1 X-ray crystal structure of 9a

Note: the sample was prepared from Dichloromethane/n-hexane $=1: 4$ system via slow evaporation; crystal structure of $9 \mathrm{a}$ is at $50 \%$ probability levels for the thermal ellipsoid plot. 
3. NMR spectra for all new compounds

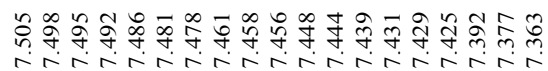

$\stackrel{m}{\stackrel{n}{r}} \underset{i}{i}$

(1)

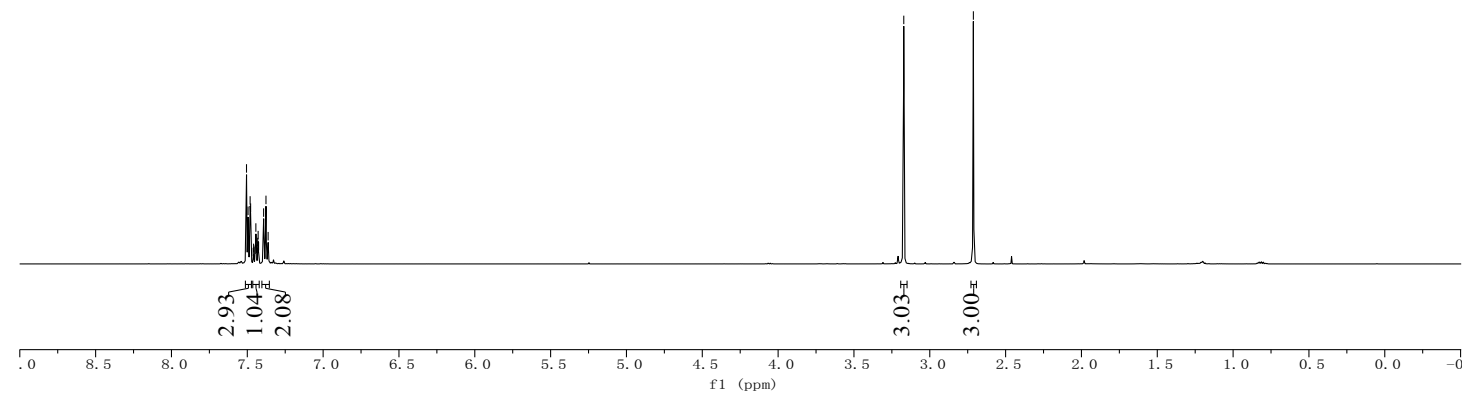

${ }^{1} \mathrm{H}$ NMR spectrum of $\mathbf{1 p}\left(500 \mathrm{MHz}, \mathrm{CDCl}_{3}\right)$

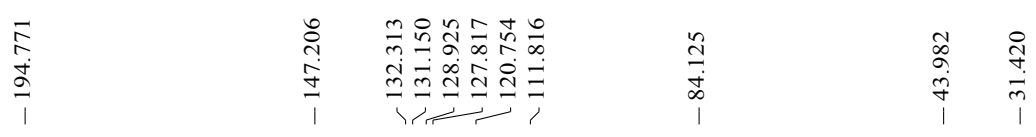
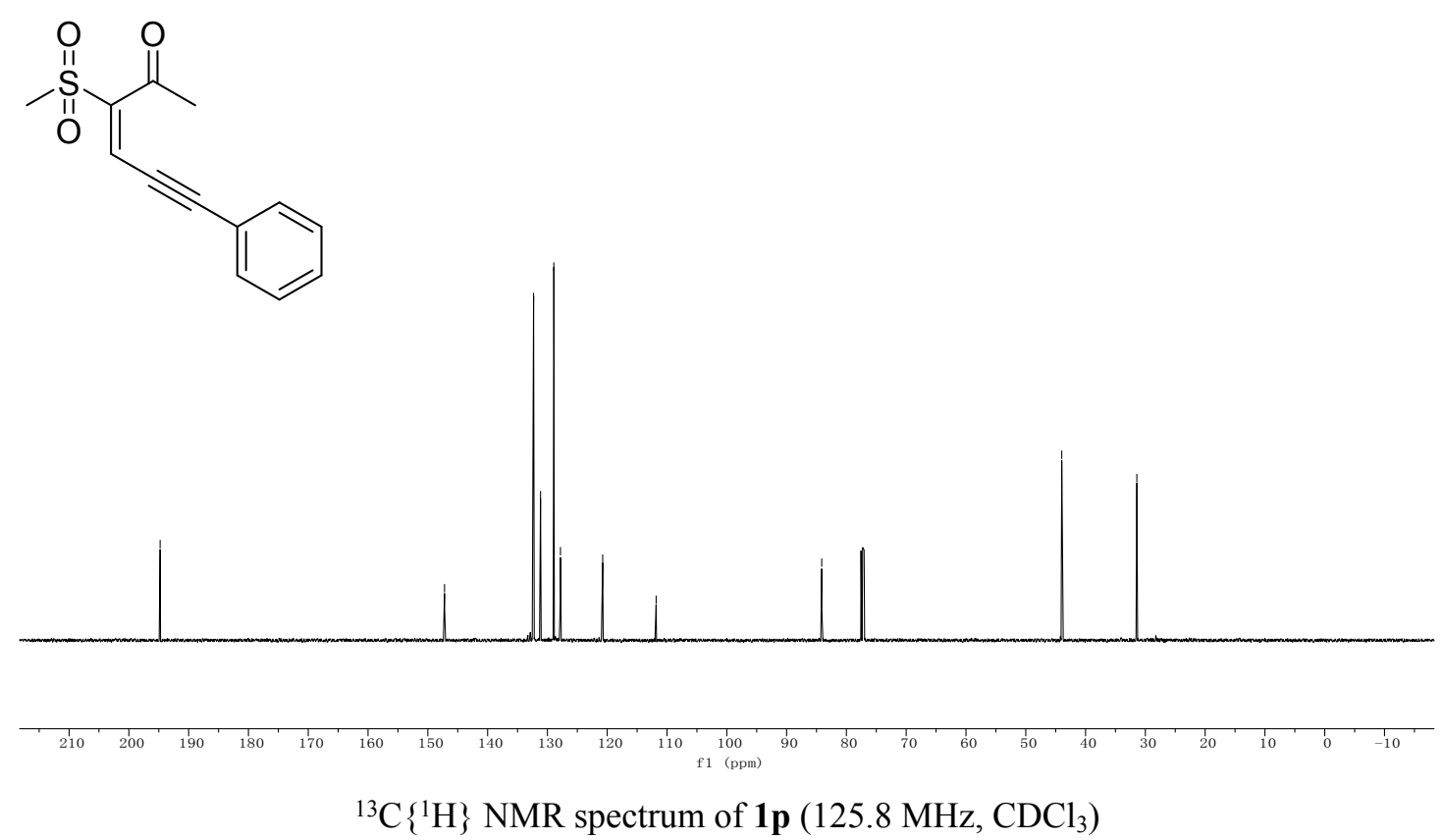
<smiles>CC(=O)C(C#Cc1ccccc1)C(C[N+](=O)[O-])C(C)=O</smiles>

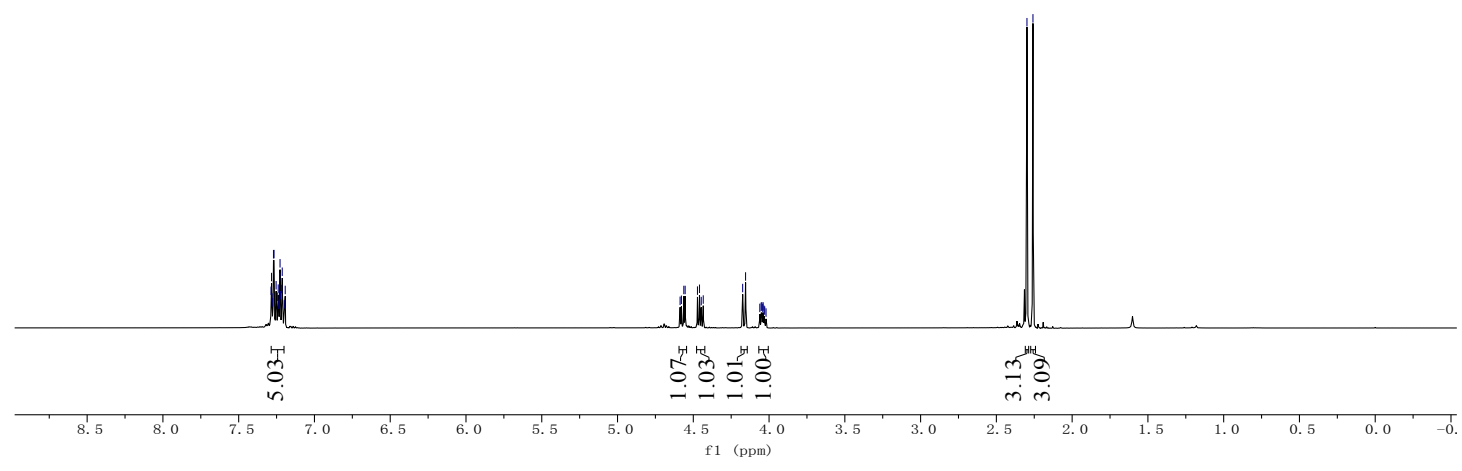

${ }^{1} \mathrm{H}$ NMR spectrum of $\mathbf{3 a}\left(500 \mathrm{MHz}, \mathrm{CDCl}_{3}\right)$<smiles>CC(=O)C(C#Cc1ccccc1)C(C[N+](=O)[O-])C(C)=O</smiles>
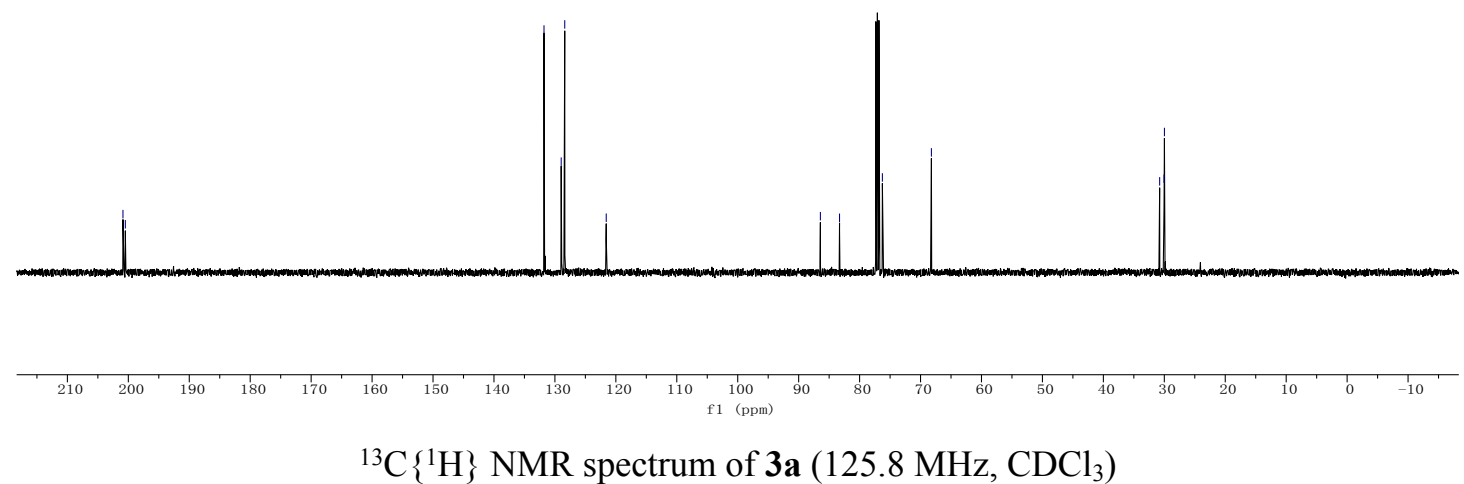

${ }^{13} \mathrm{C}\left\{{ }^{1} \mathrm{H}\right\}$ NMR spectrum of $\mathbf{3 a}\left(125.8 \mathrm{MHz}, \mathrm{CDCl}_{3}\right)$ 
<smiles>CC(=O)C1=C(C)O/C(=C\c2ccccc2)C1C[N+](=O)[O-]</smiles>

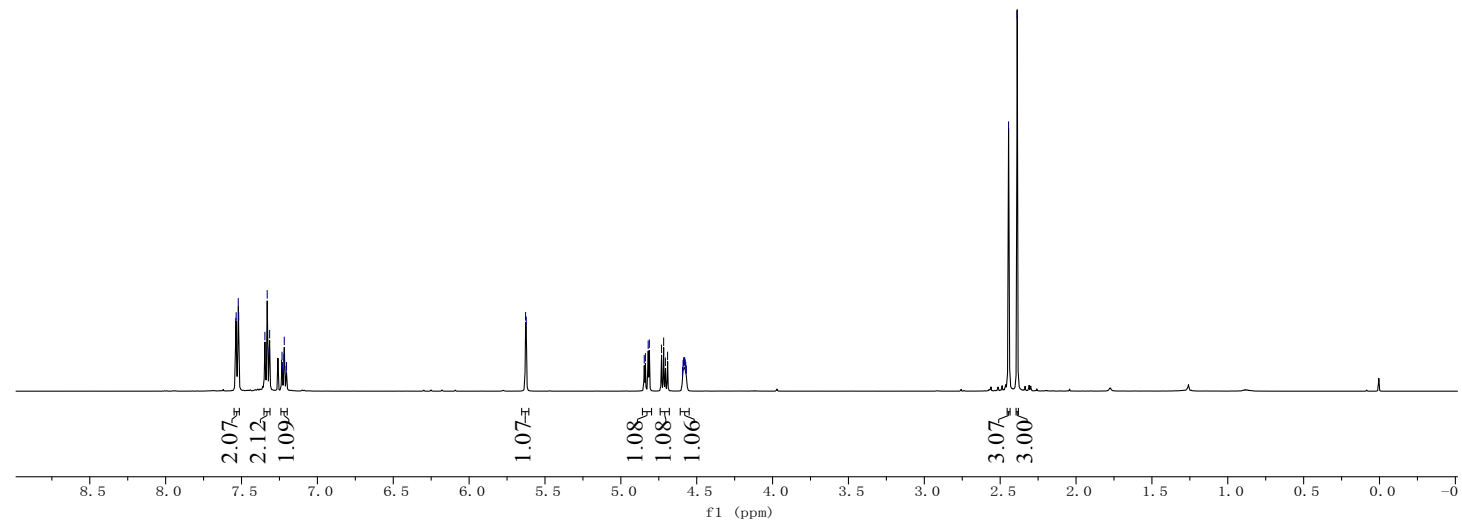

${ }^{1} \mathrm{H}$ NMR spectrum of $4 a\left(500 \mathrm{MHz}, \mathrm{CDCl}_{3}\right)$<smiles>CC(=O)C1=C(C)O/C(=C\c2ccccc2)C1C[N+](=O)[O-]</smiles>
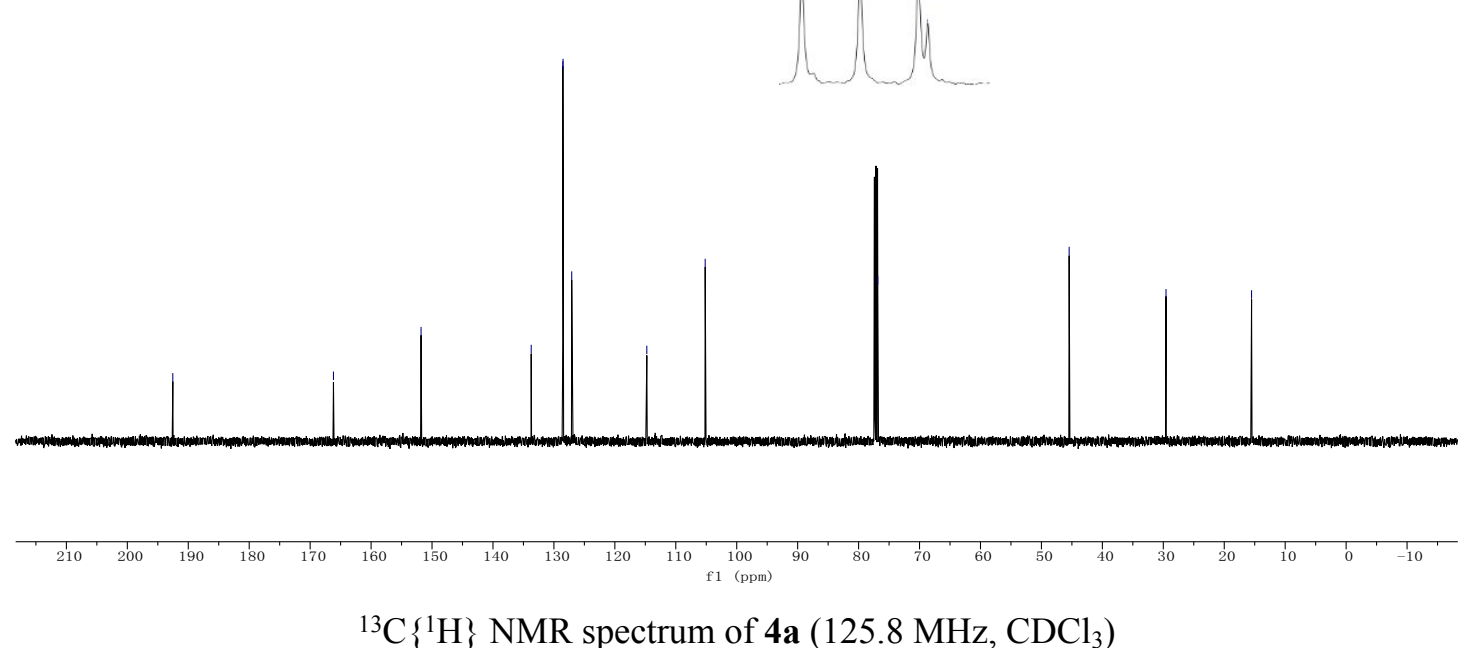

${ }^{13} \mathrm{C}\left\{{ }^{1} \mathrm{H}\right\}$ NMR spectrum of $\mathbf{4 a}\left(125.8 \mathrm{MHz}, \mathrm{CDCl}_{3}\right)$ 
<smiles>COc1ccccc1</smiles>

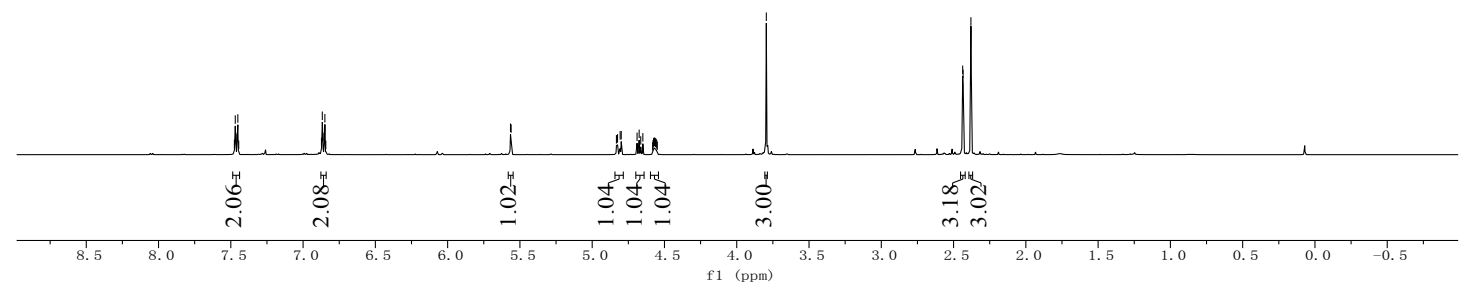

${ }^{1} \mathrm{H}$ NMR spectrum of $\mathbf{4 d}\left(500 \mathrm{MHz}, \mathrm{CDCl}_{3}\right)$

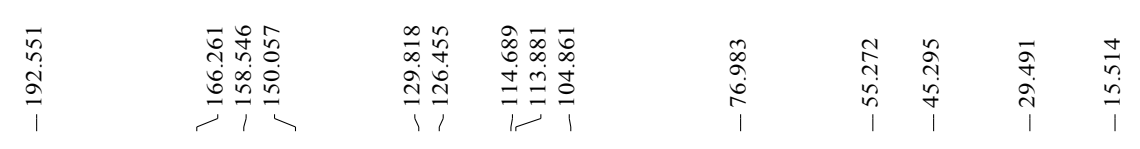<smiles>COc1ccccc1</smiles>
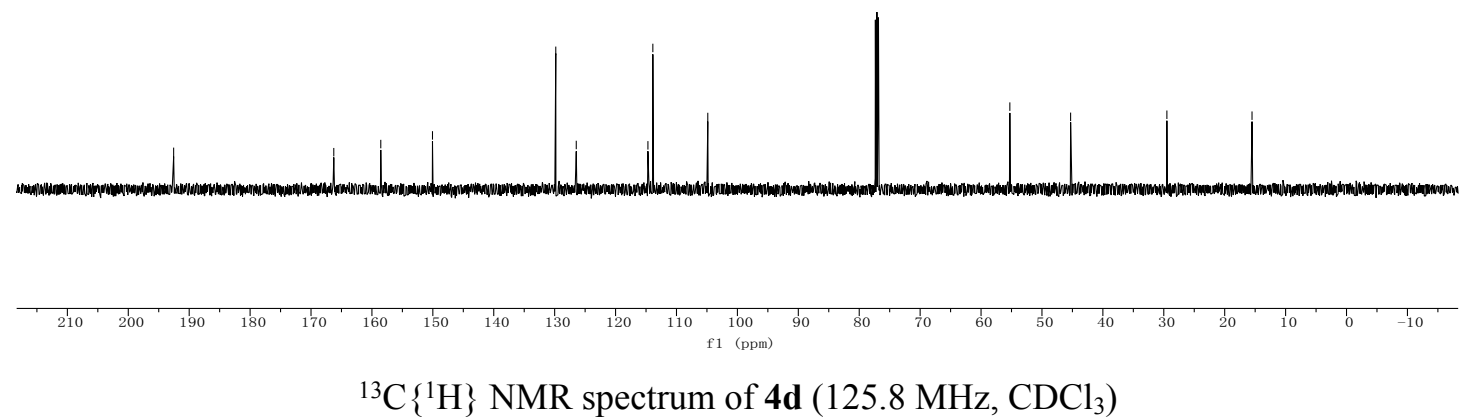

${ }^{13} \mathrm{C}\left\{{ }^{1} \mathrm{H}\right\}$ NMR spectrum of $\mathbf{4 d}\left(125.8 \mathrm{MHz}, \mathrm{CDCl}_{3}\right)$ 

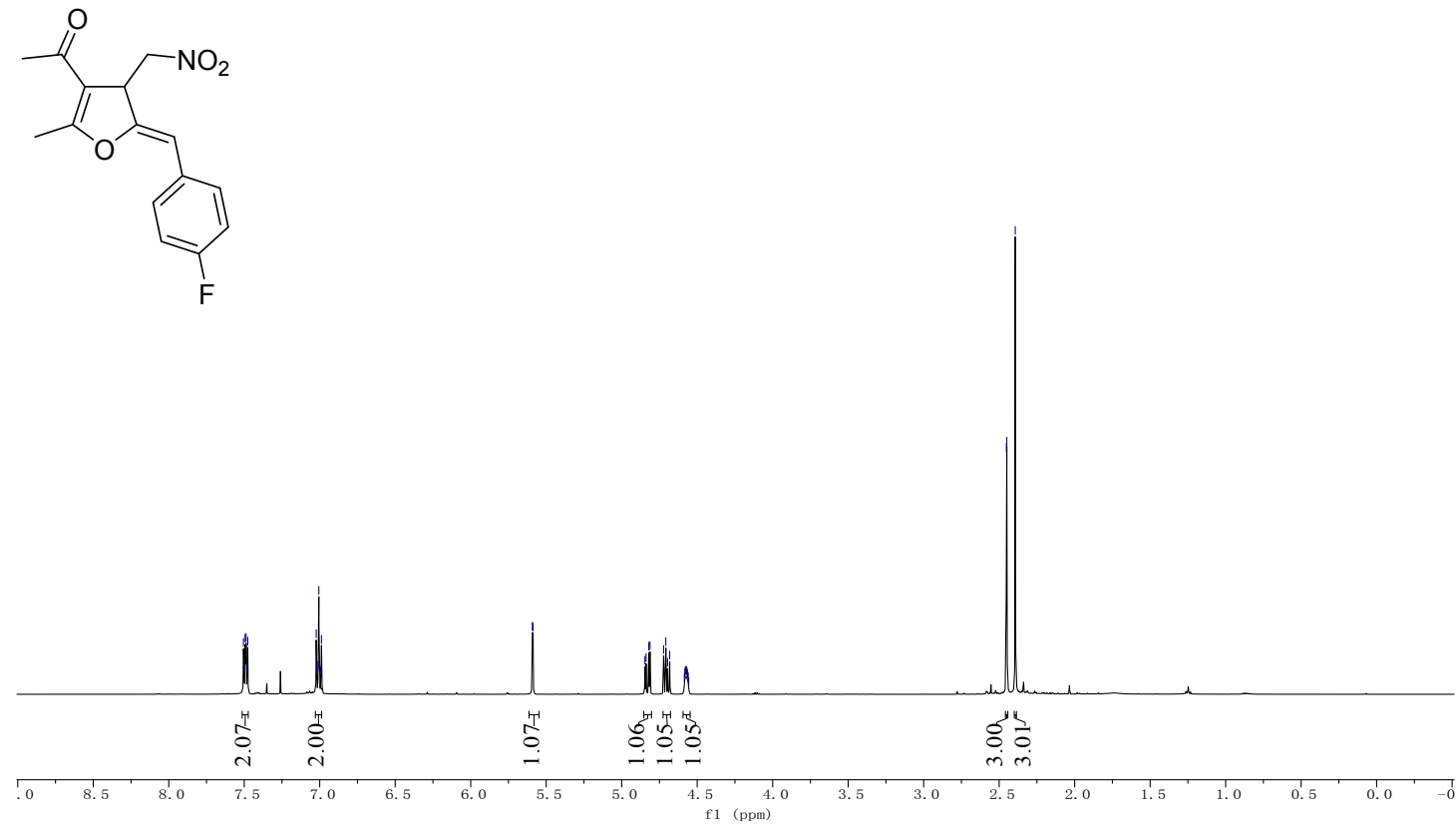

${ }^{1} \mathrm{H}$ NMR spectrum of $\mathbf{4 g}\left(500 \mathrm{MHz}, \mathrm{CDCl}_{3}\right)$

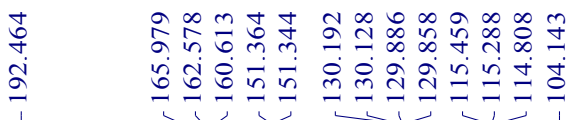

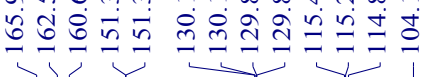

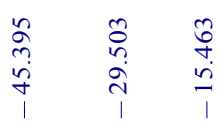<smiles>CC(=O)C1=C(C)O/C(=C\c2ccc(F)cc2)C1C[N+](=O)[O-]</smiles>
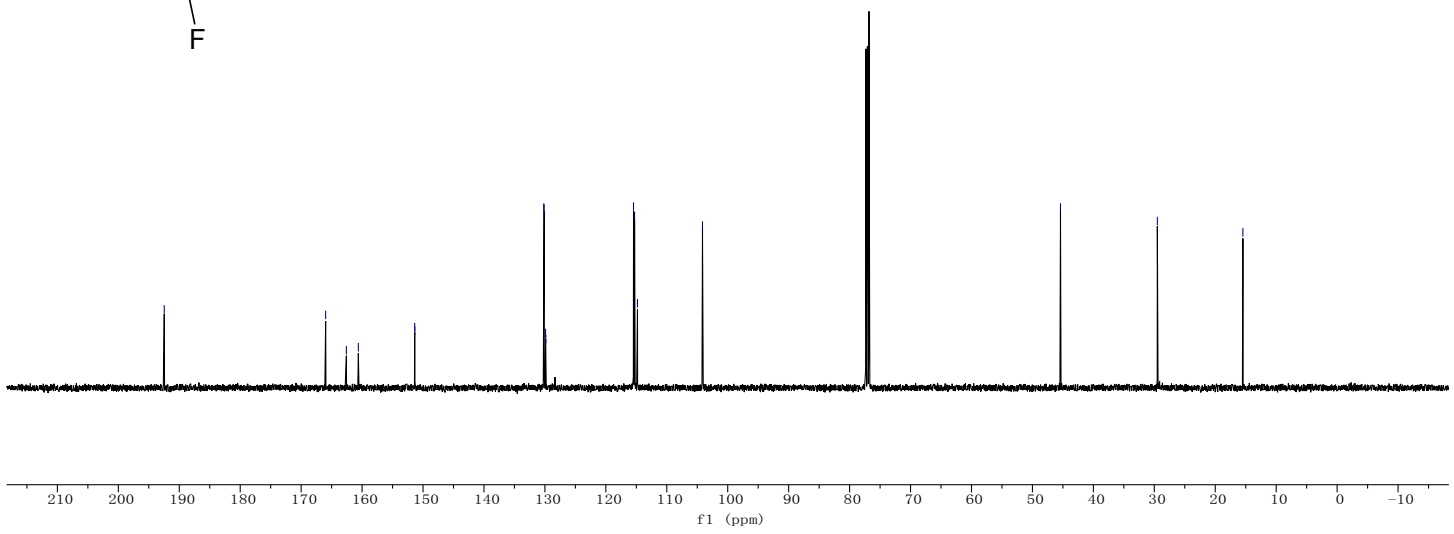

${ }^{13} \mathrm{C}\left\{{ }^{1} \mathrm{H}\right\}$ NMR spectrum of $\mathbf{4 g}\left(125.8 \mathrm{MHz}, \mathrm{CDCl}_{3}\right)$ 

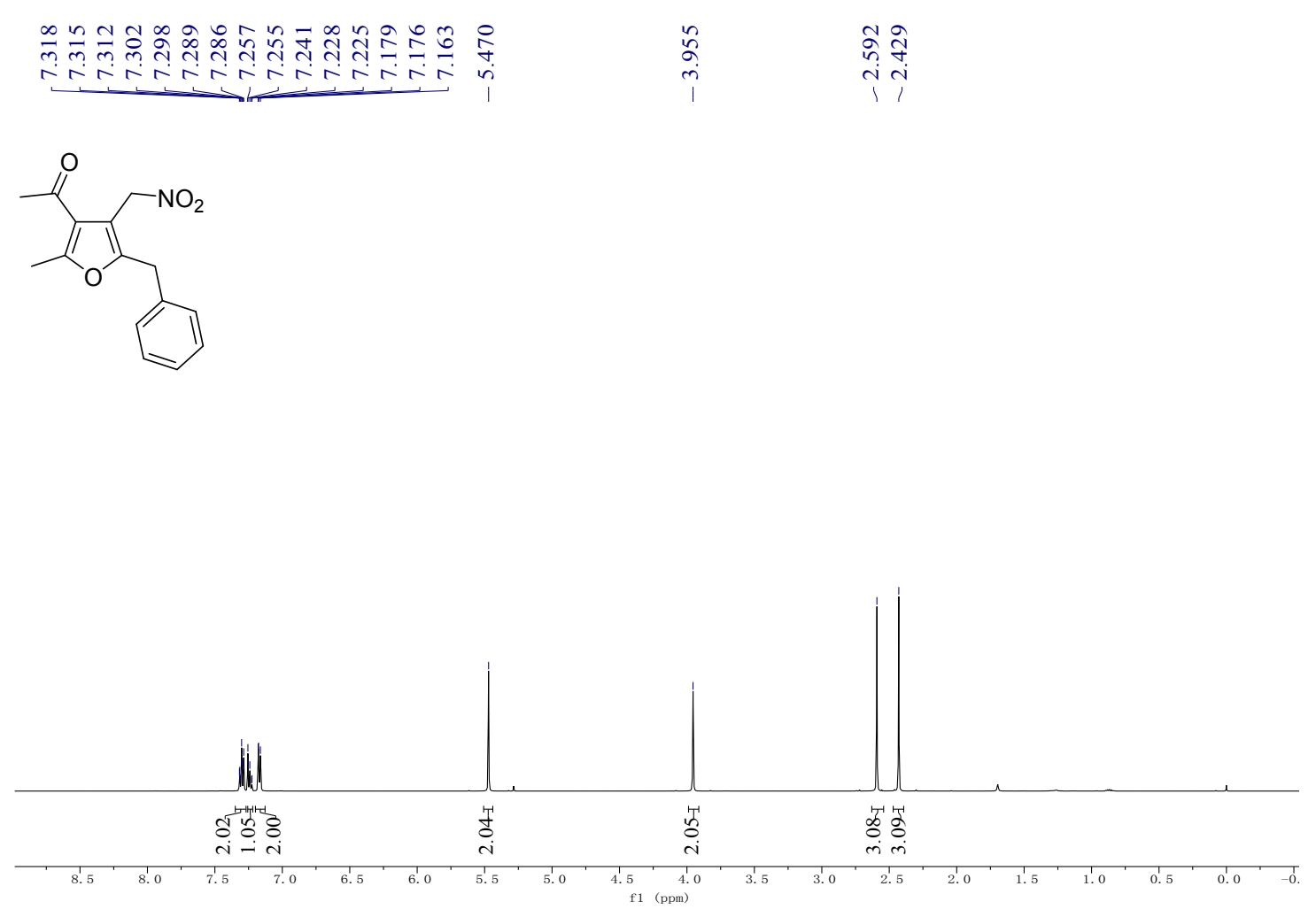

${ }^{1} \mathrm{H}$ NMR spectrum of $\mathbf{5 a}\left(500 \mathrm{MHz}, \mathrm{CDCl}_{3}\right)$

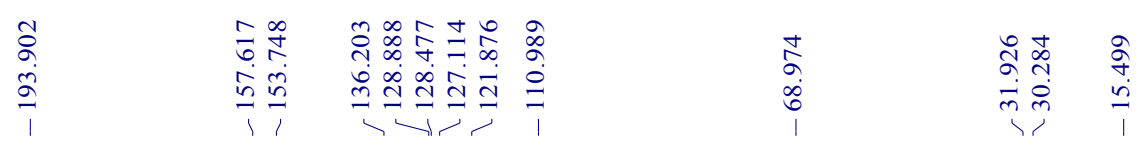<smiles>CC(=O)c1c(C)oc(Cc2ccccc2)c1C[N+](=O)[O-]</smiles>

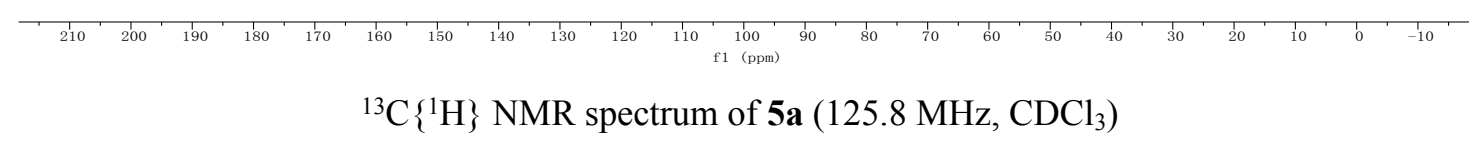




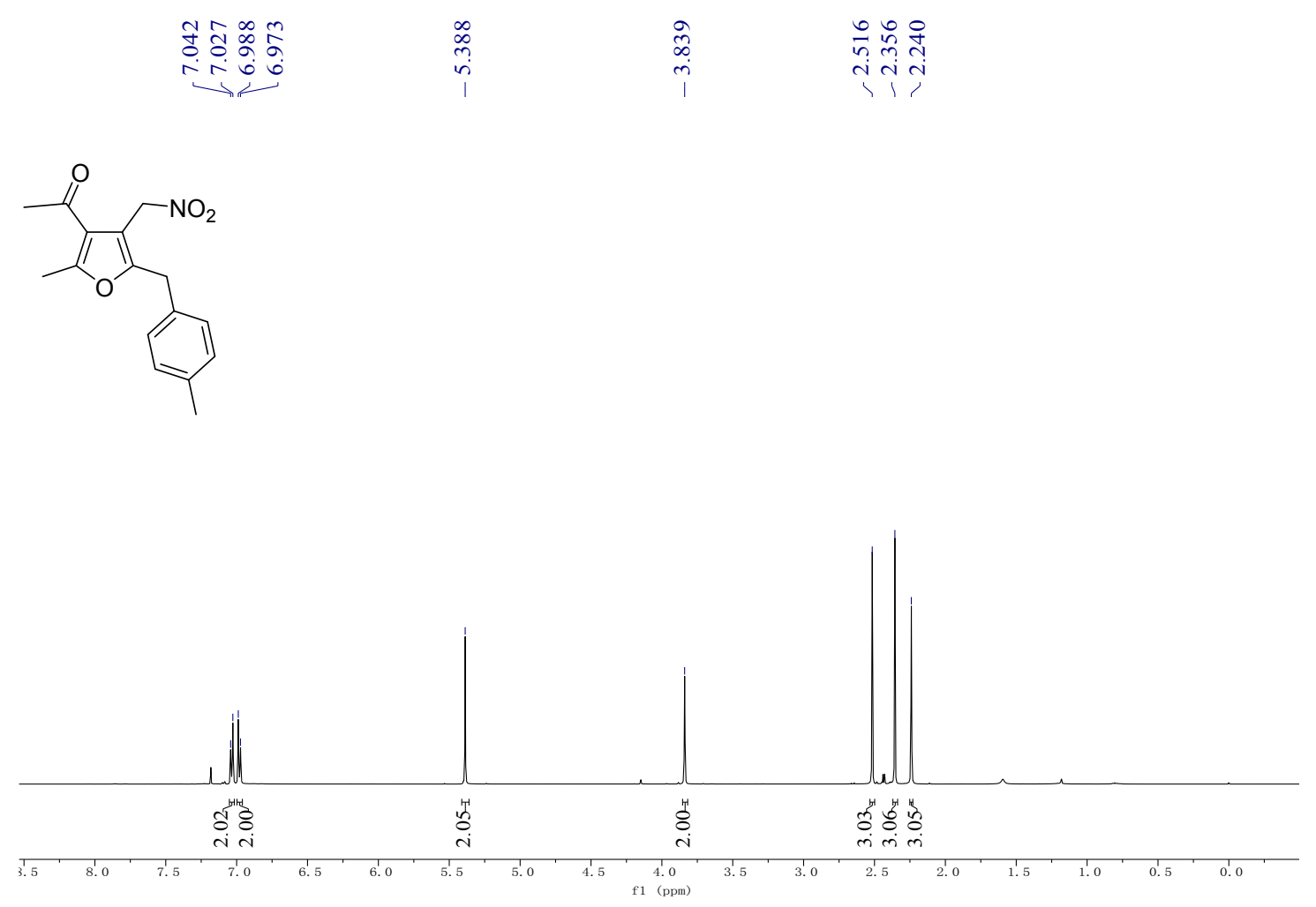

${ }^{1} \mathrm{H}$ NMR spectrum of $\mathbf{5 b}\left(500 \mathrm{MHz}, \mathrm{CDCl}_{3}\right)$

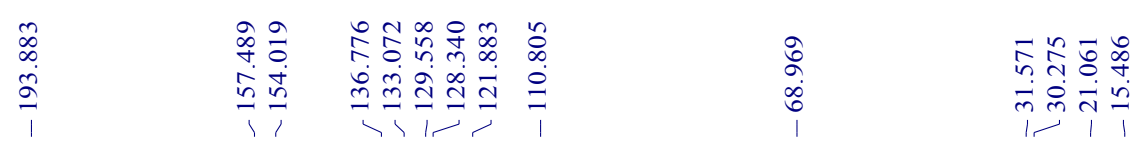<smiles>CC(=O)c1c(C)oc(Cc2ccc(C)cc2)c1C[N+](=O)[O-]</smiles>
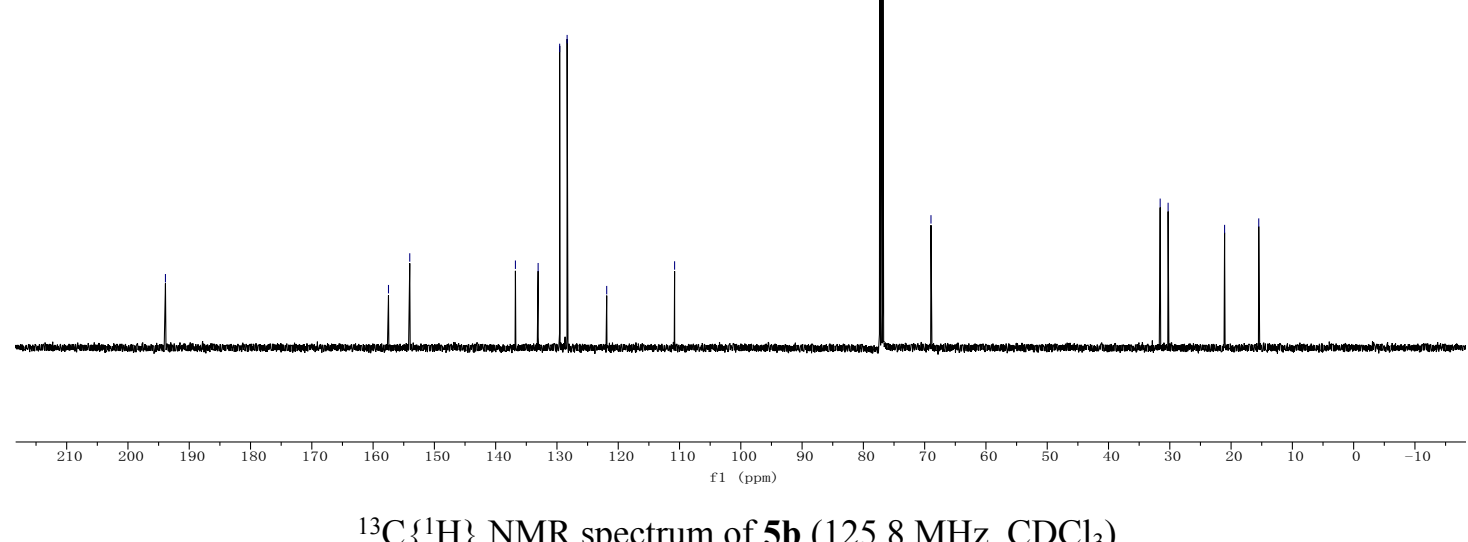

${ }^{13} \mathrm{C}\left\{{ }^{1} \mathrm{H}\right\}$ NMR spectrum of $\mathbf{5 b}\left(125.8 \mathrm{MHz}, \mathrm{CDCl}_{3}\right)$ 


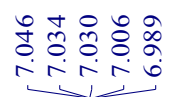

$\underset{\substack{\infty \\ \infty \\ i}}{i}$
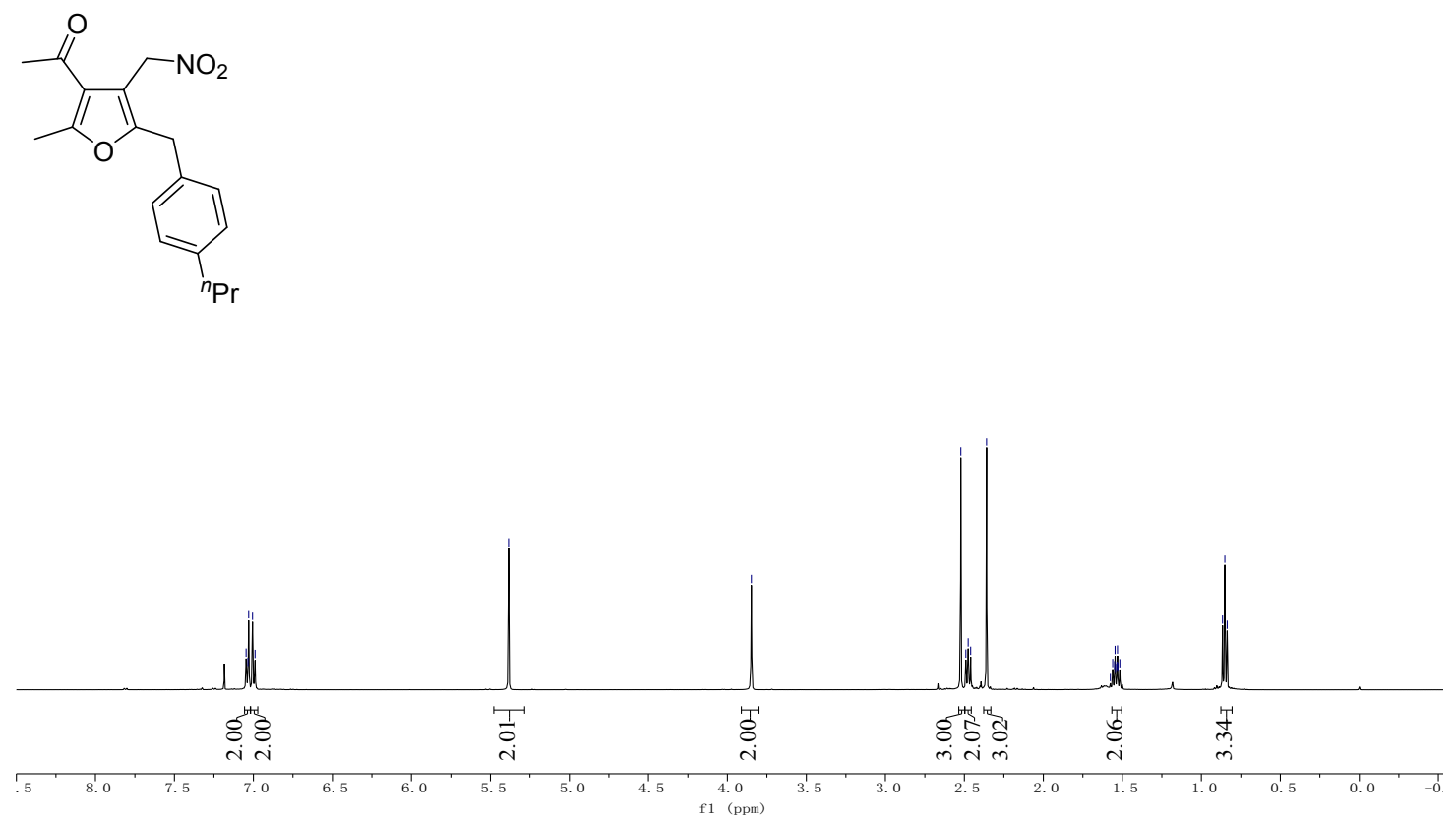

${ }^{1} \mathrm{H}$ NMR spectrum of $\mathbf{5 c}\left(500 \mathrm{MHz}, \mathrm{CDCl}_{3}\right)$

$\overrightarrow{0}$
$\infty$
$\stackrel{9}{a}$

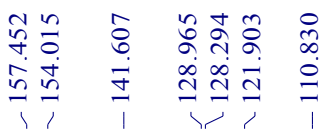

$n$
2
$\dot{0}$
$\dot{0}$

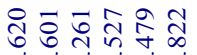

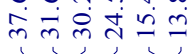<smiles>CC(=O)c1c(C)oc(Cc2ccc(C(C)C)cc2)c1C[N+](=O)[O-]</smiles>
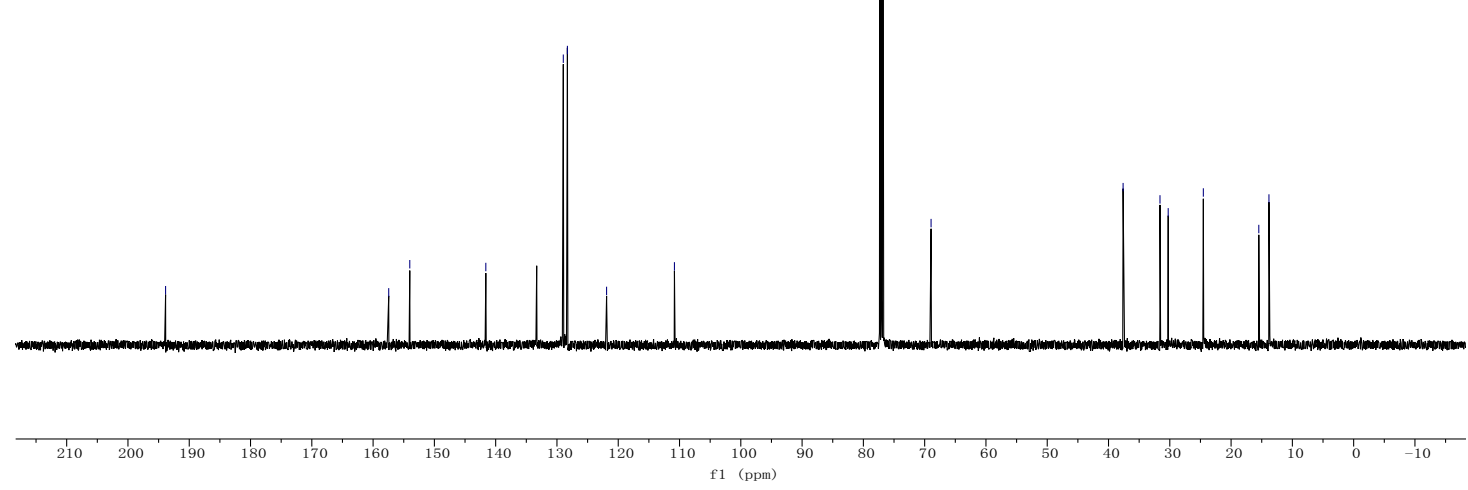

${ }^{13} \mathrm{C}\left\{{ }^{1} \mathrm{H}\right\}$ NMR spectrum of $\mathbf{5 c}\left(125.8 \mathrm{MHz}, \mathrm{CDCl}_{3}\right)$ 


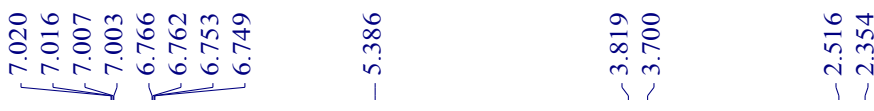<smiles>COc1ccc(Cc2oc(C)c(C(C)=O)c2C[N+](=O)[O-])cc1</smiles>

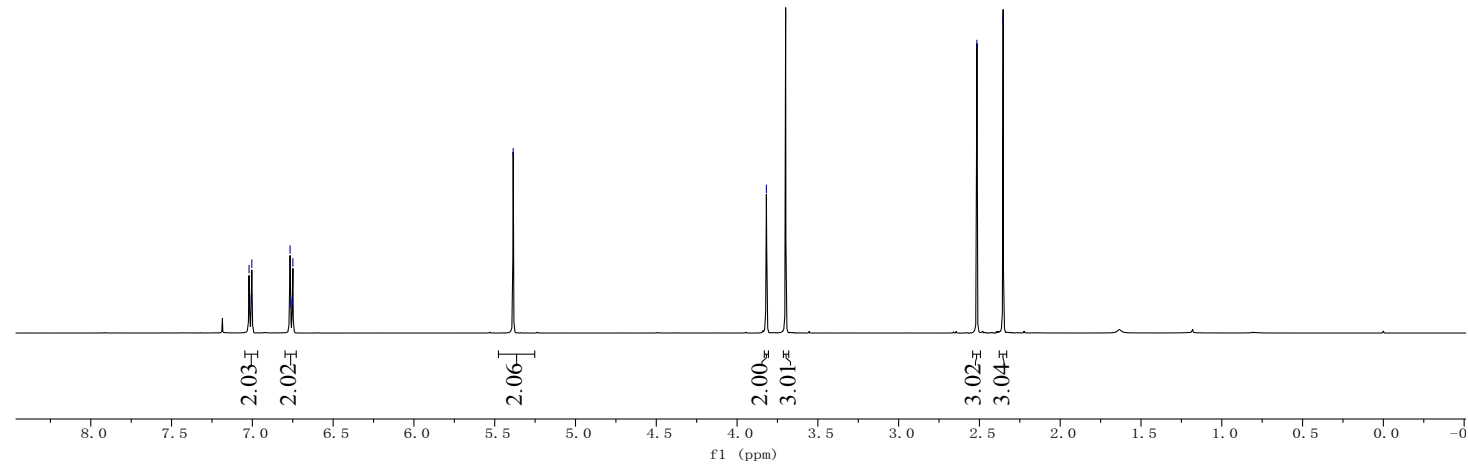

${ }^{1} \mathrm{H}$ NMR spectrum of $\mathbf{5 d}\left(500 \mathrm{MHz}, \mathrm{CDCl}_{3}\right)$

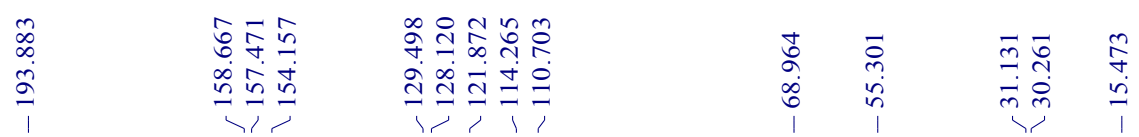
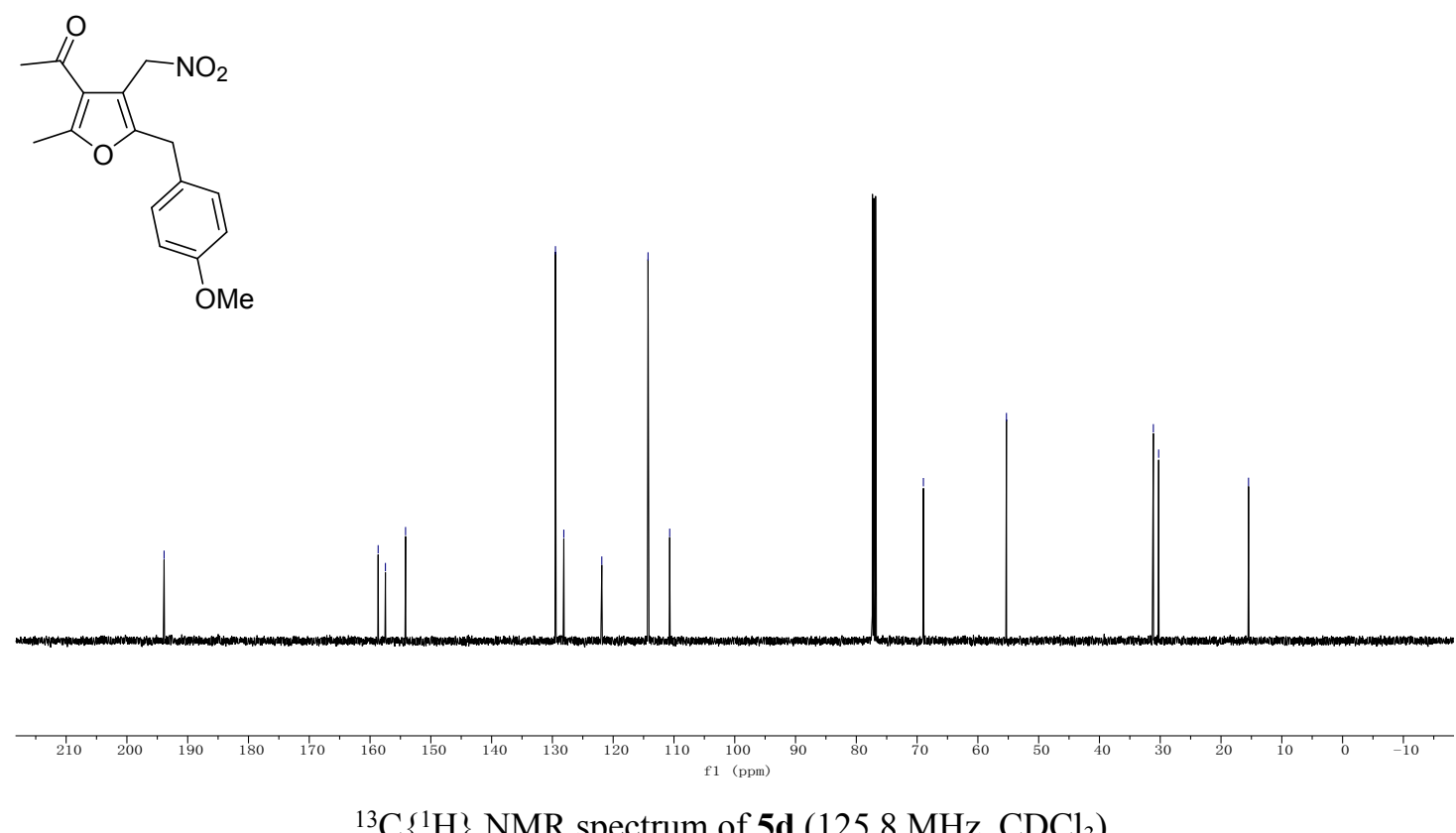

${ }^{13} \mathrm{C}\left\{{ }^{1} \mathrm{H}\right\}$ NMR spectrum of $\mathbf{5 d}\left(125.8 \mathrm{MHz}, \mathrm{CDCl}_{3}\right)$ 


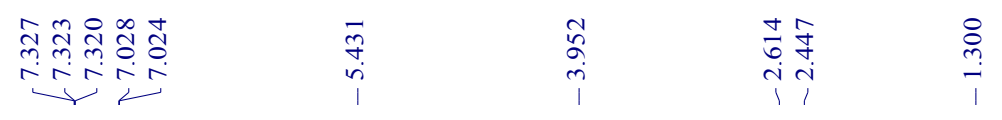
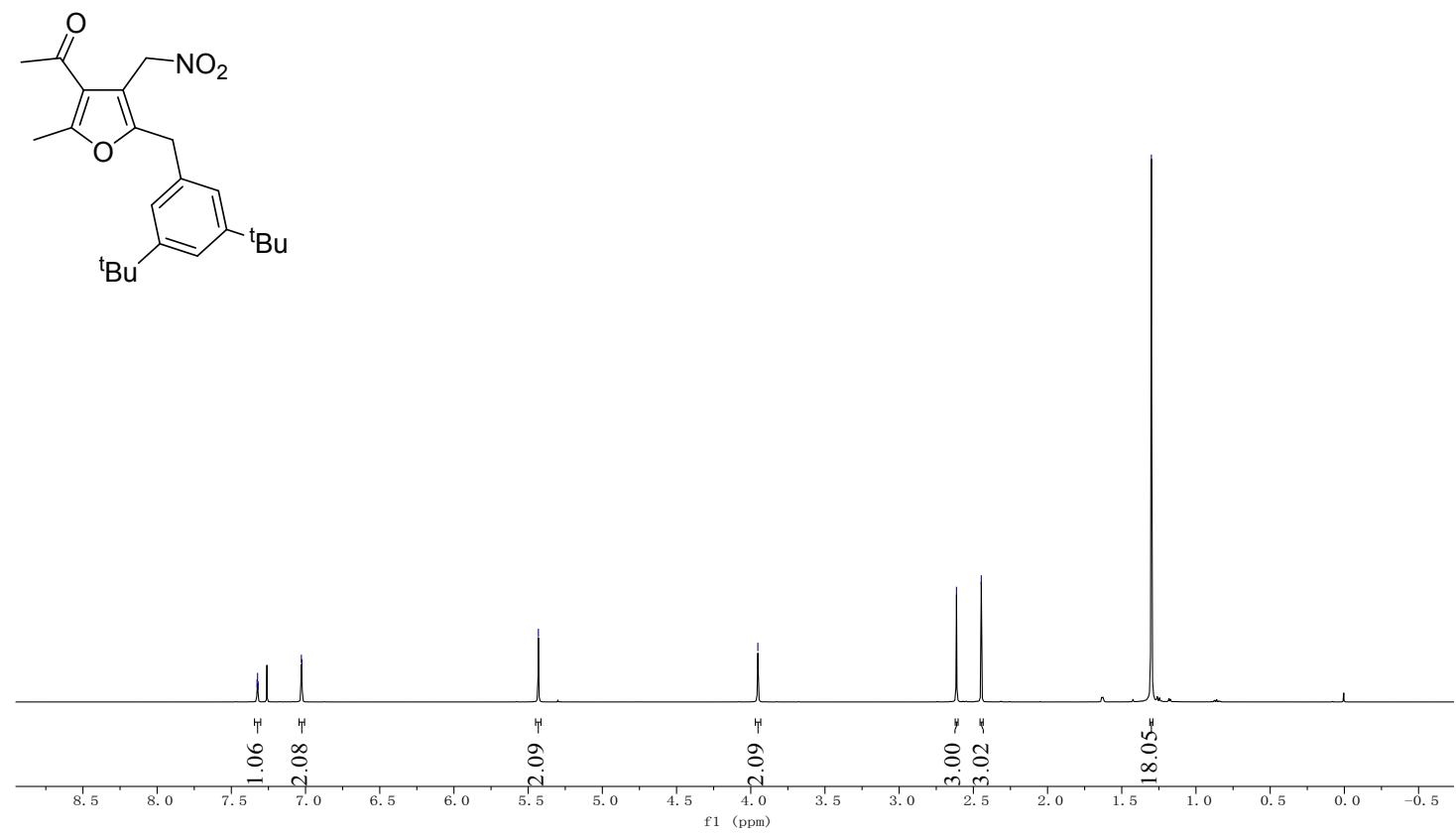

${ }^{1} \mathrm{H}$ NMR spectrum of $\mathbf{5 e}\left(500 \mathrm{MHz}, \mathrm{CDCl}_{3}\right)$

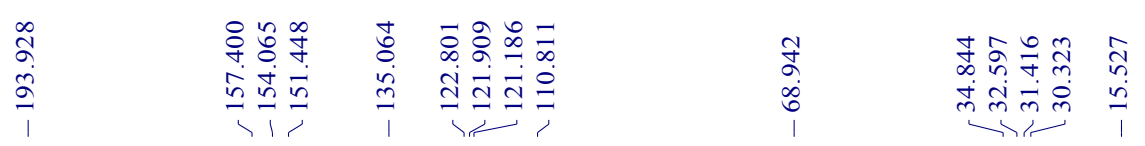
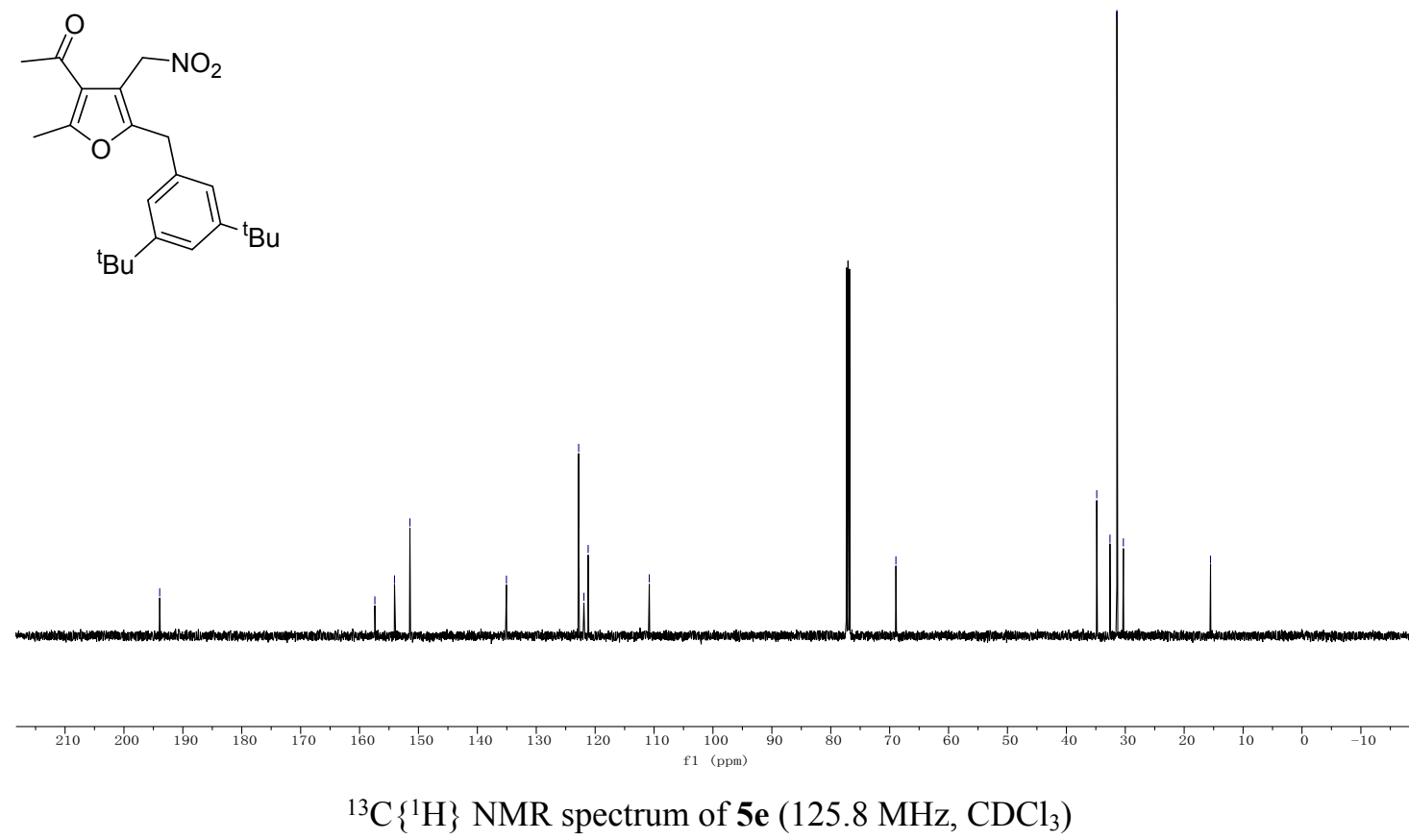


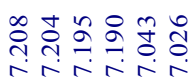

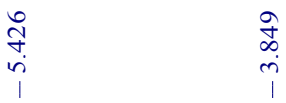

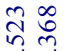

$\begin{array}{ll}1 & 1 \\ 4 & 1\end{array}$<smiles>CC(=O)c1c(C)oc(Cc2ccc(Cl)cc2)c1C[N+](=O)[O-]</smiles>

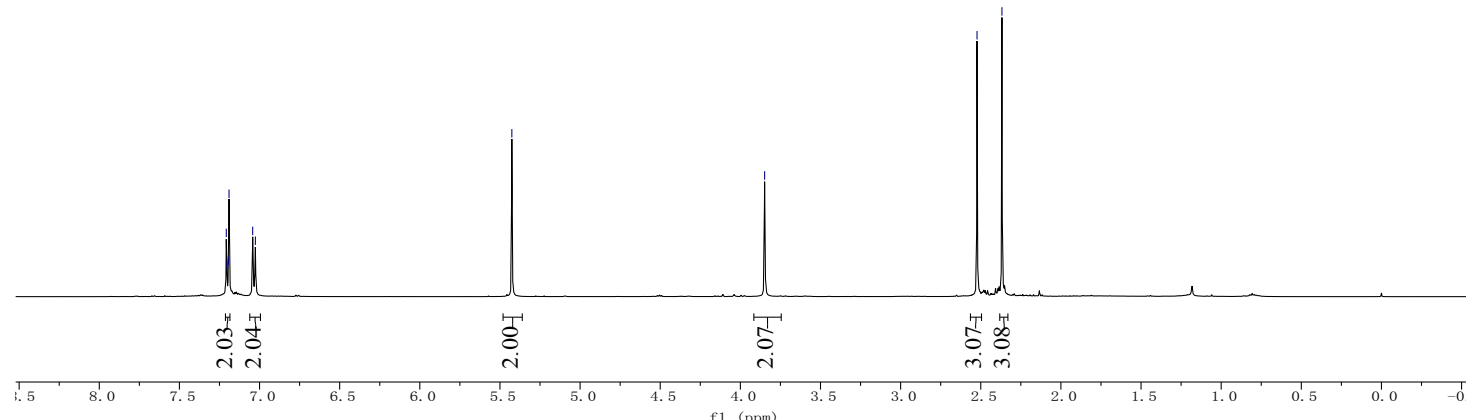

${ }^{1} \mathrm{H}$ NMR spectrum of $\mathbf{5 f}\left(500 \mathrm{MHz}, \mathrm{CDCl}_{3}\right)$

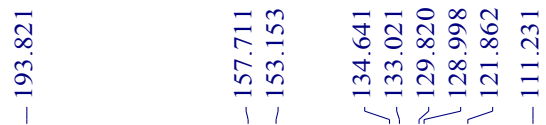

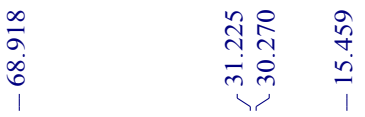<smiles>CC(=O)c1c(C)oc(Cc2ccc(Cl)cc2)c1C[N+](=O)[O-]</smiles>
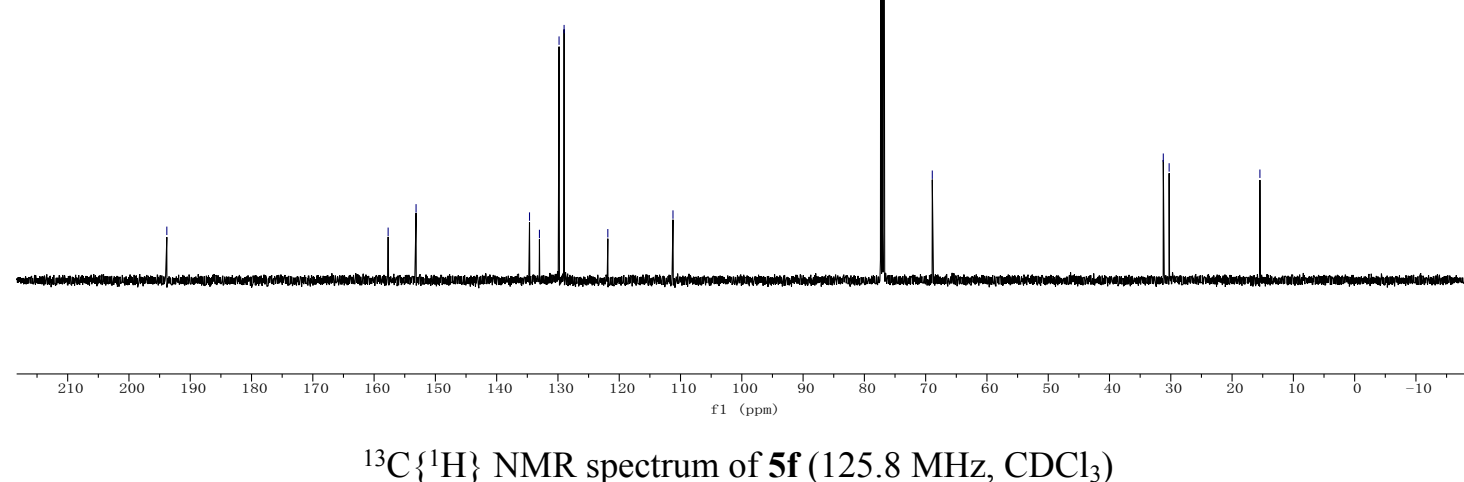

${ }^{13} \mathrm{C}\left\{{ }^{1} \mathrm{H}\right\}$ NMR spectrum of $\mathbf{5 f}\left(125.8 \mathrm{MHz}, \mathrm{CDCl}_{3}\right)$ 
<smiles>CC(=O)c1c(C)oc(Cc2ccc(F)cc2)c1C[N+](=O)[O-]</smiles>

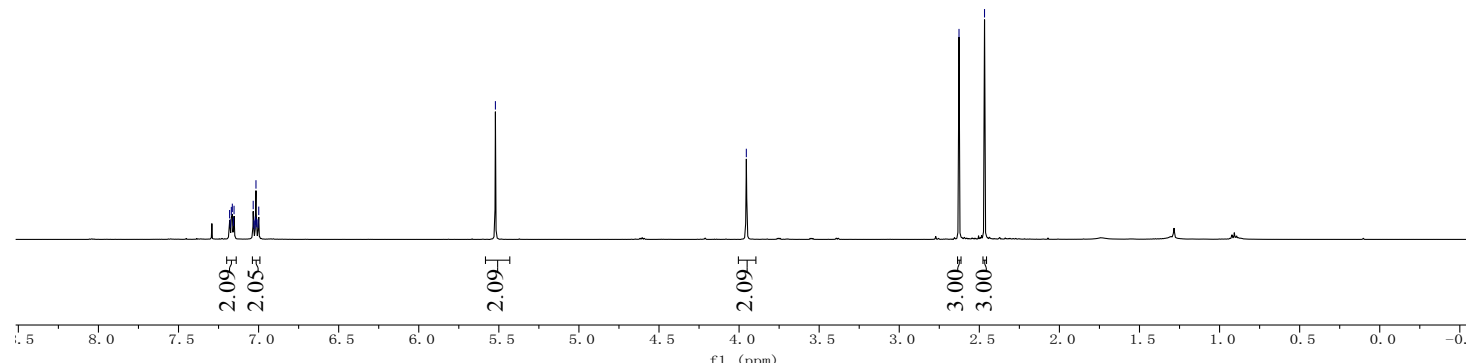

${ }^{1} \mathrm{H}$ NMR spectrum of $\mathbf{5 g}\left(500 \mathrm{MHz}, \mathrm{CDCl}_{3}\right)$

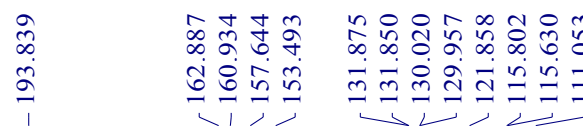

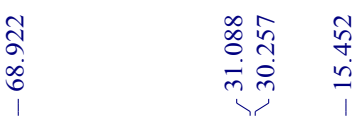<smiles>CC(=O)c1c(C)oc(Cc2ccc(F)cc2)c1C[N+](=O)[O-]</smiles>
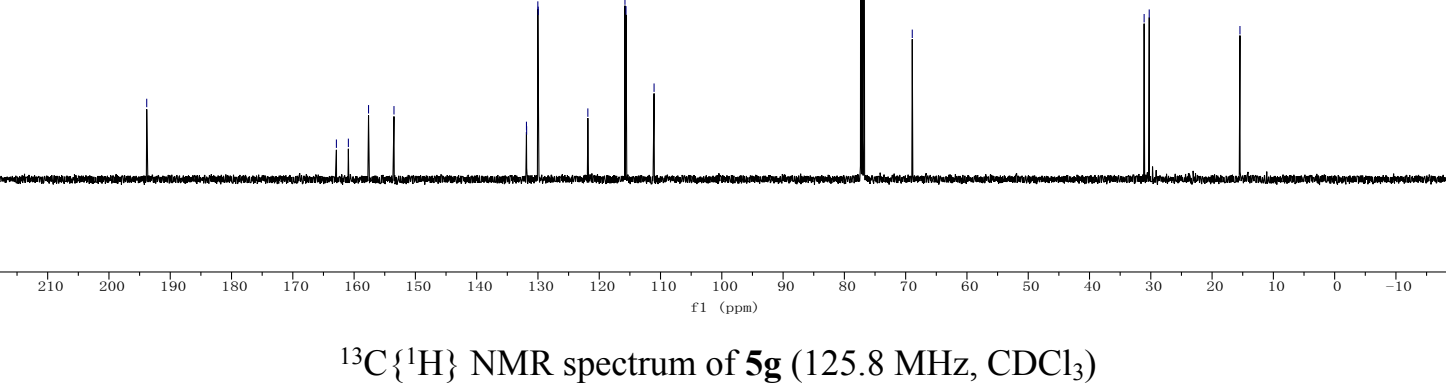
<smiles>CC(=O)c1c(C)oc(CC2CC2)c1[N+](=O)[O-]</smiles>

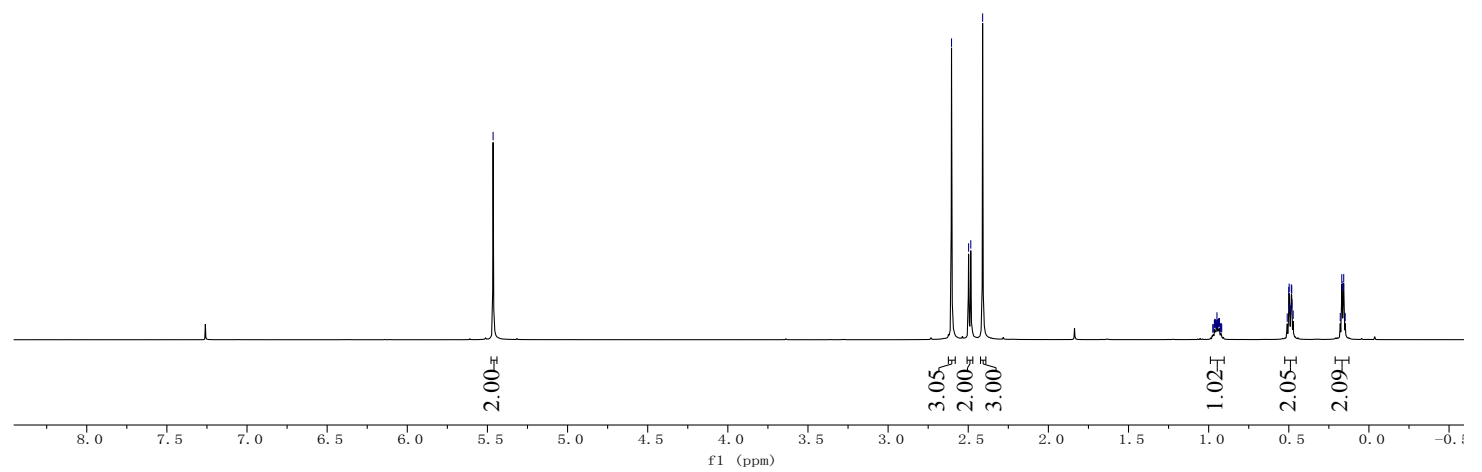

${ }^{1} \mathrm{H}$ NMR spectrum of $\mathbf{5 h}\left(500 \mathrm{MHz}, \mathrm{CDCl}_{3}\right)$

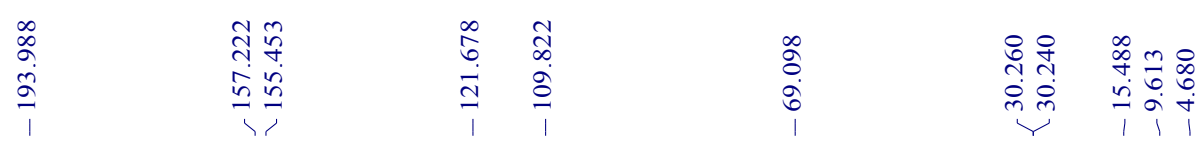<smiles>CC(=O)c1c(C)oc(CC2CC2)c1[N+](=O)[O-]</smiles>
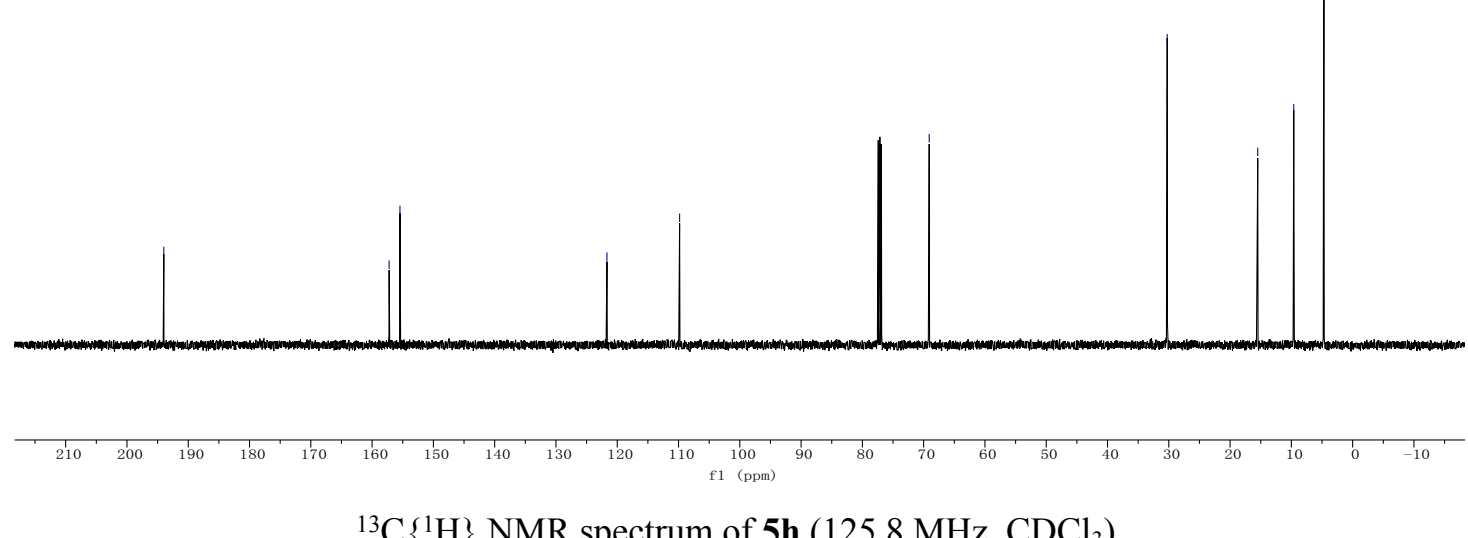

${ }^{13} \mathrm{C}\left\{{ }^{1} \mathrm{H}\right\}$ NMR spectrum of $\mathbf{5 h}\left(125.8 \mathrm{MHz}, \mathrm{CDCl}_{3}\right)$ 

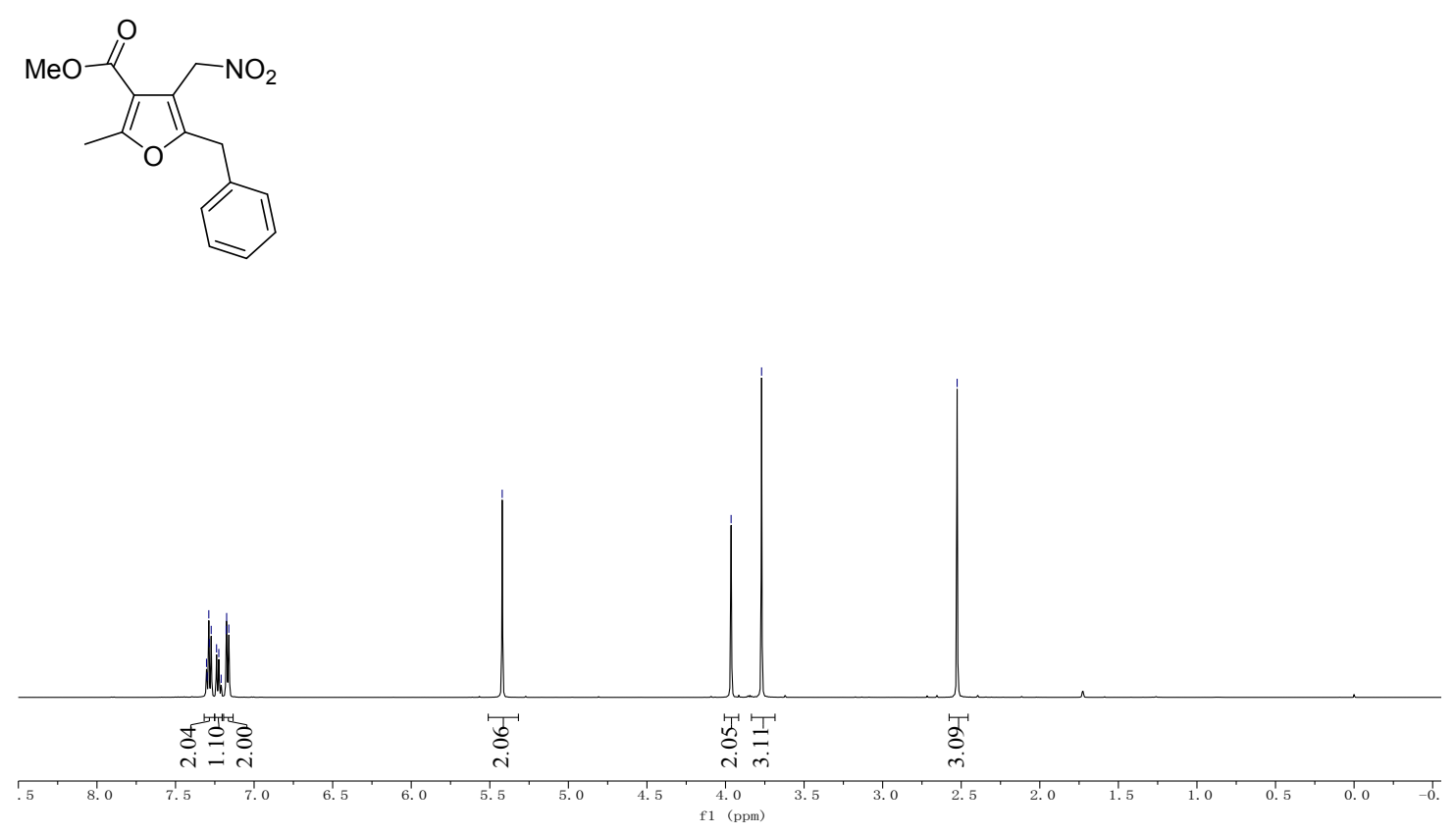

${ }^{1} \mathrm{H}$ NMR spectrum of $\mathbf{5 i}\left(500 \mathrm{MHz}, \mathrm{CDCl}_{3}\right)$

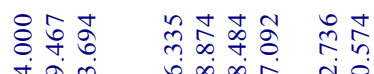

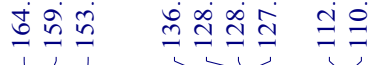

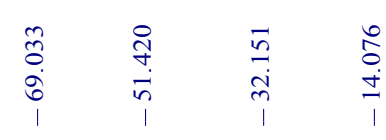
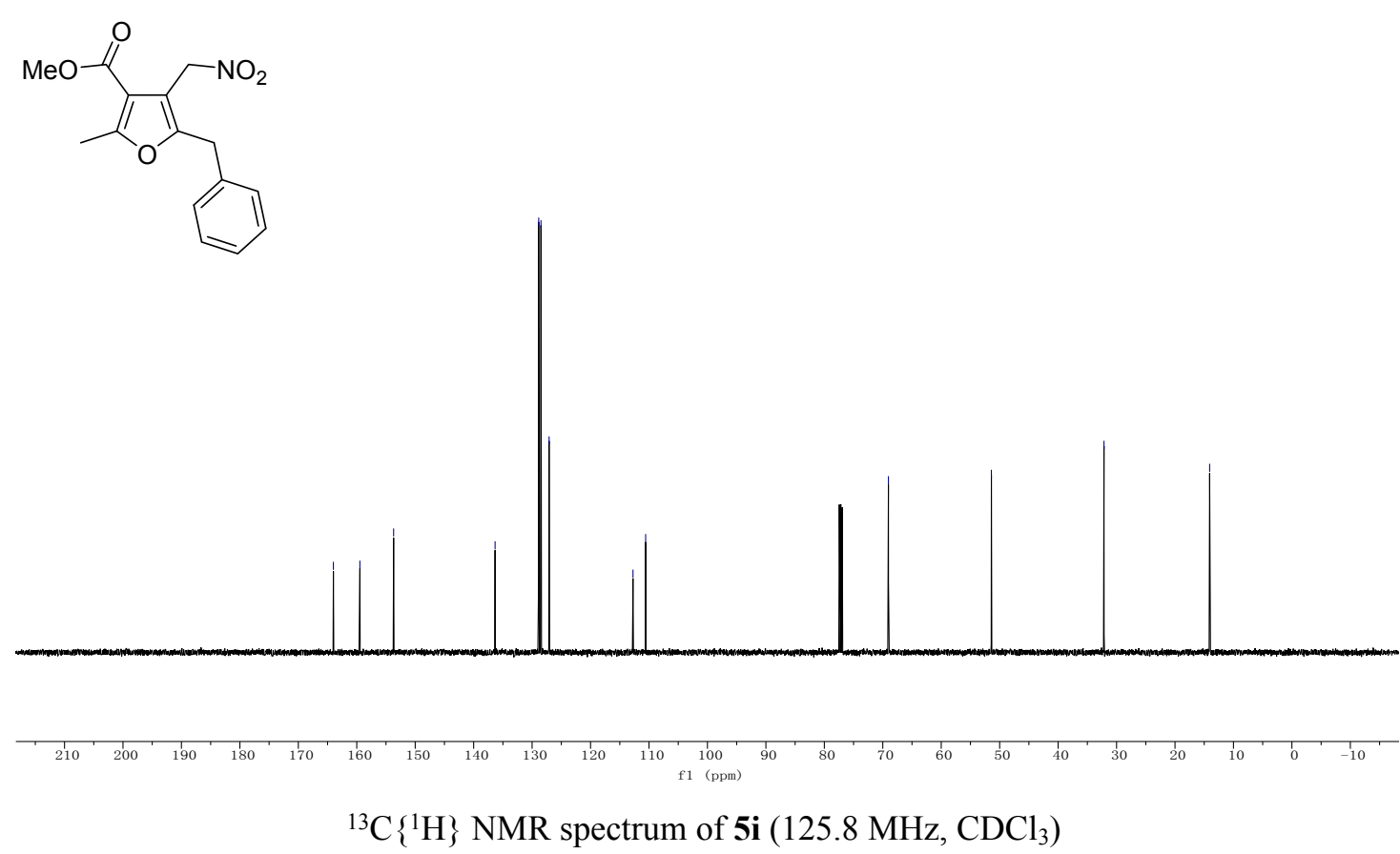


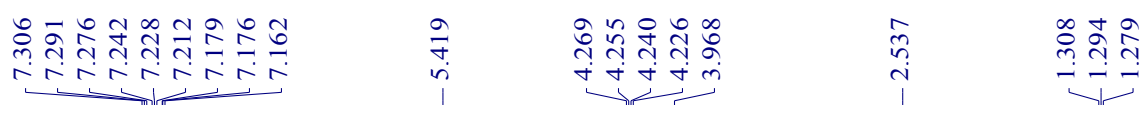<smiles>CCOC(=O)c1c(C)oc(Cc2ccccc2)c1C[N+](=O)[O-]</smiles>

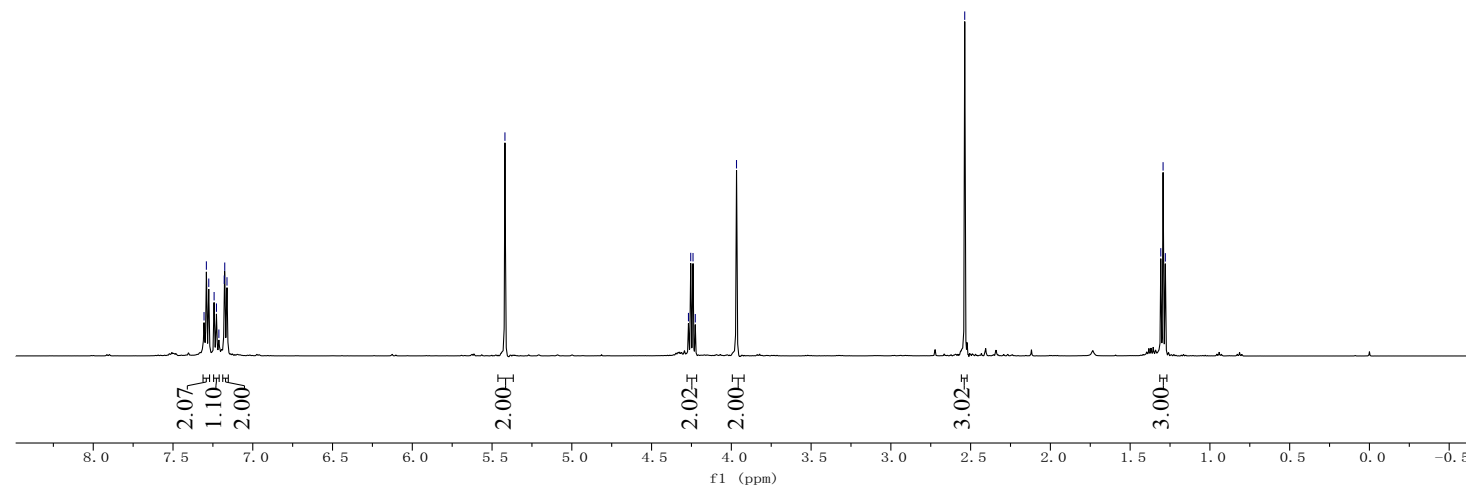

${ }^{1} \mathrm{H}$ NMR spectrum of $\mathbf{5 j}\left(500 \mathbf{M H z}, \mathrm{CDCl}_{3}\right)$

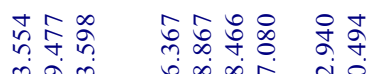

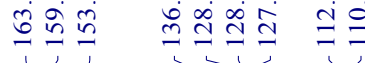

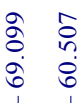

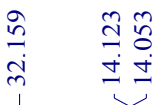<smiles>CCOC(=O)c1c(C)oc(Cc2ccccc2)c1C[N+](=O)[O-]</smiles>

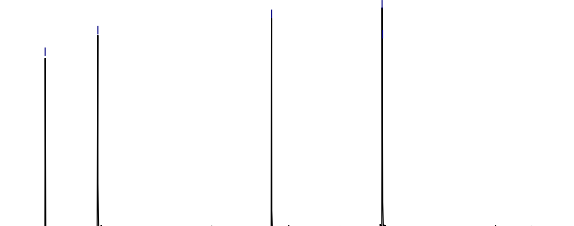



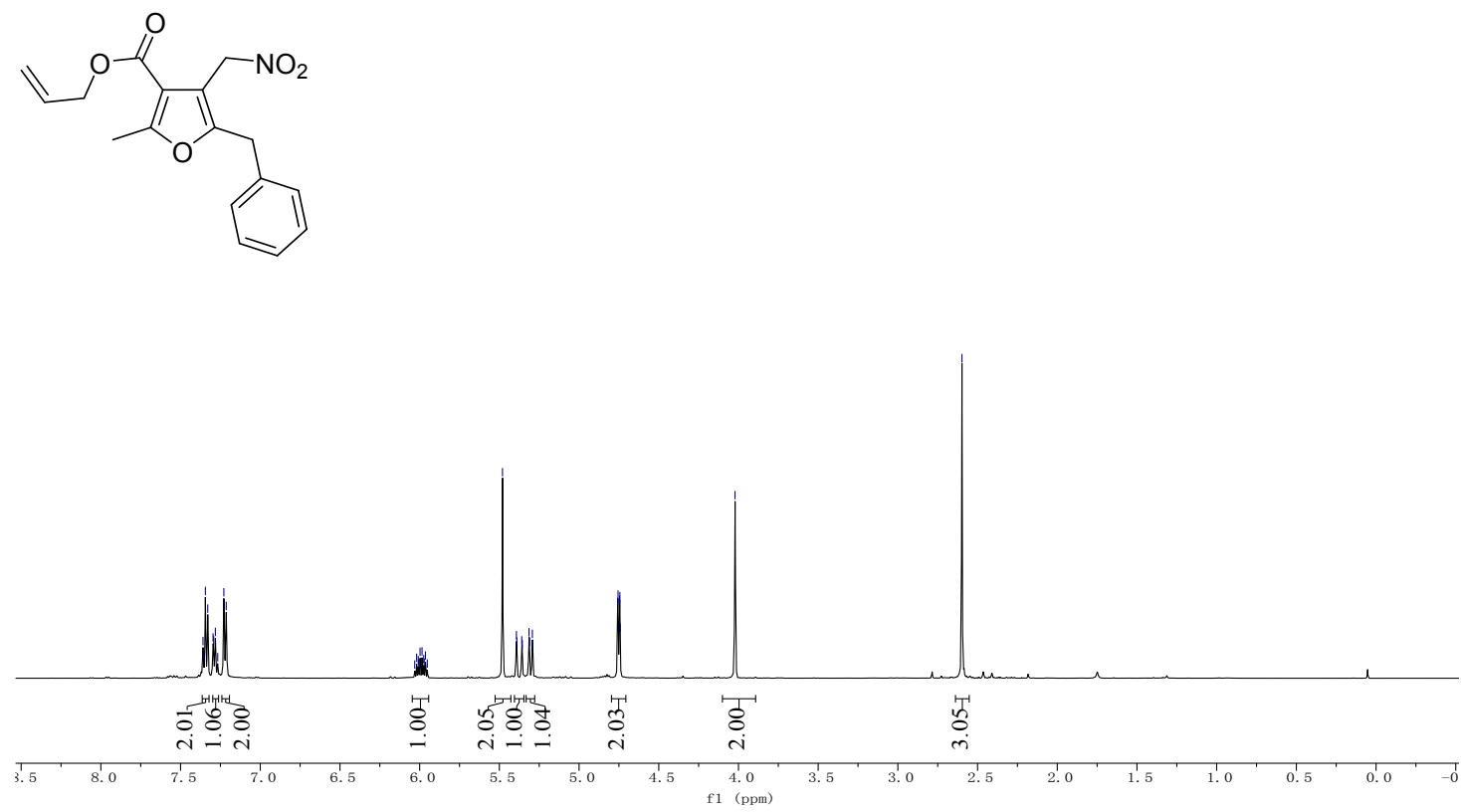

${ }^{1} \mathrm{H}$ NMR spectrum of $\mathbf{5 k}\left(500 \mathrm{MHz}, \mathrm{CDCl}_{3}\right)$

तु)

흔

$\frac{m}{2} \frac{1}{2}$

ติ่

$\stackrel{\infty}{\infty} \quad \frac{n}{2}$
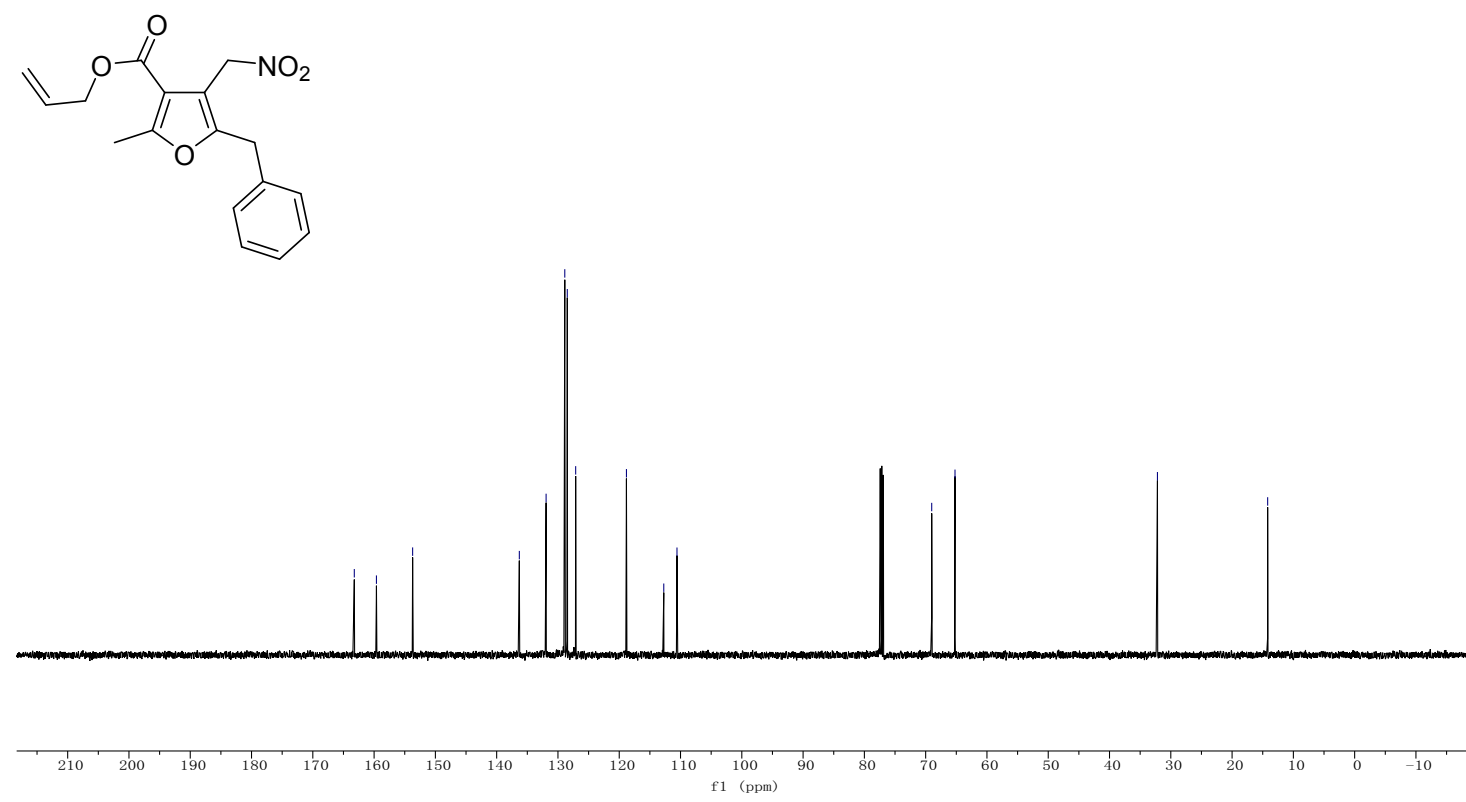

${ }^{13} \mathrm{C}\left\{{ }^{1} \mathrm{H}\right\}$ NMR spectrum of $\mathbf{5 k}\left(125.8 \mathrm{MHz}, \mathrm{CDCl}_{3}\right)$ 
<smiles>Cc1oc(Cc2ccccc2)c(C[N+](=O)[O-])c1C(=O)OCc1ccccc1</smiles>

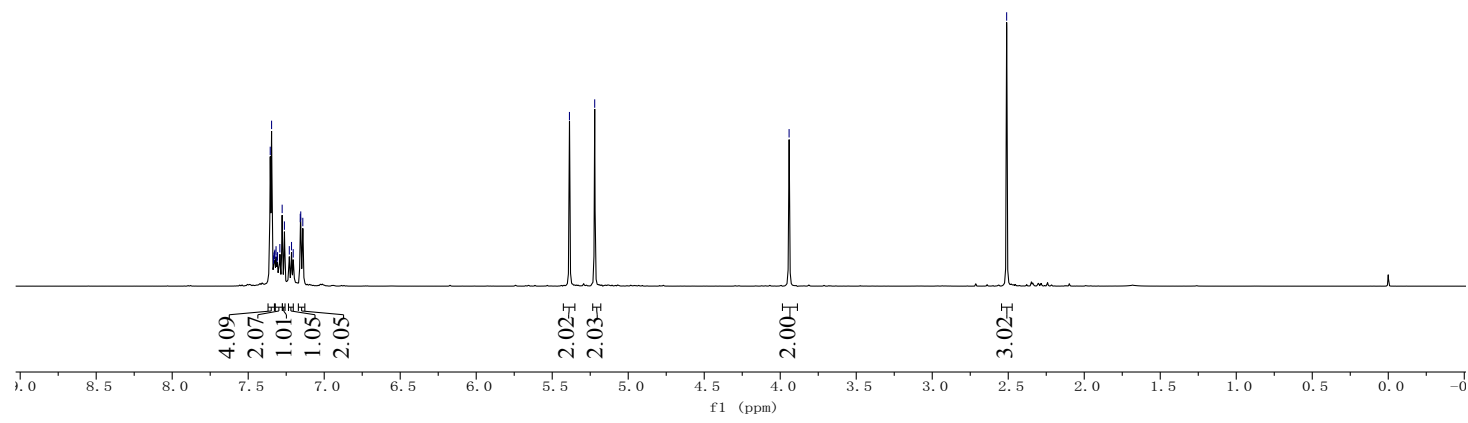

${ }^{1} \mathrm{H}$ NMR spectrum of $\mathbf{5 l}\left(500 \mathrm{MHz}, \mathrm{CDCl}_{3}\right)$

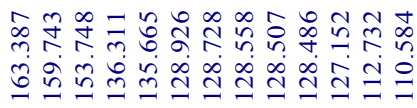

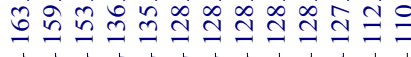

$\begin{array}{ll}1 & \\ 2 & \infty \\ 0 & 0 \\ 0 & 0 \\ 0 & 1\end{array}$

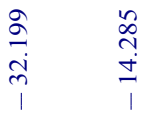<smiles>Cc1oc(Cc2ccccc2)c(C[N+](=O)[O-])c1C(=O)OCc1ccccc1</smiles>
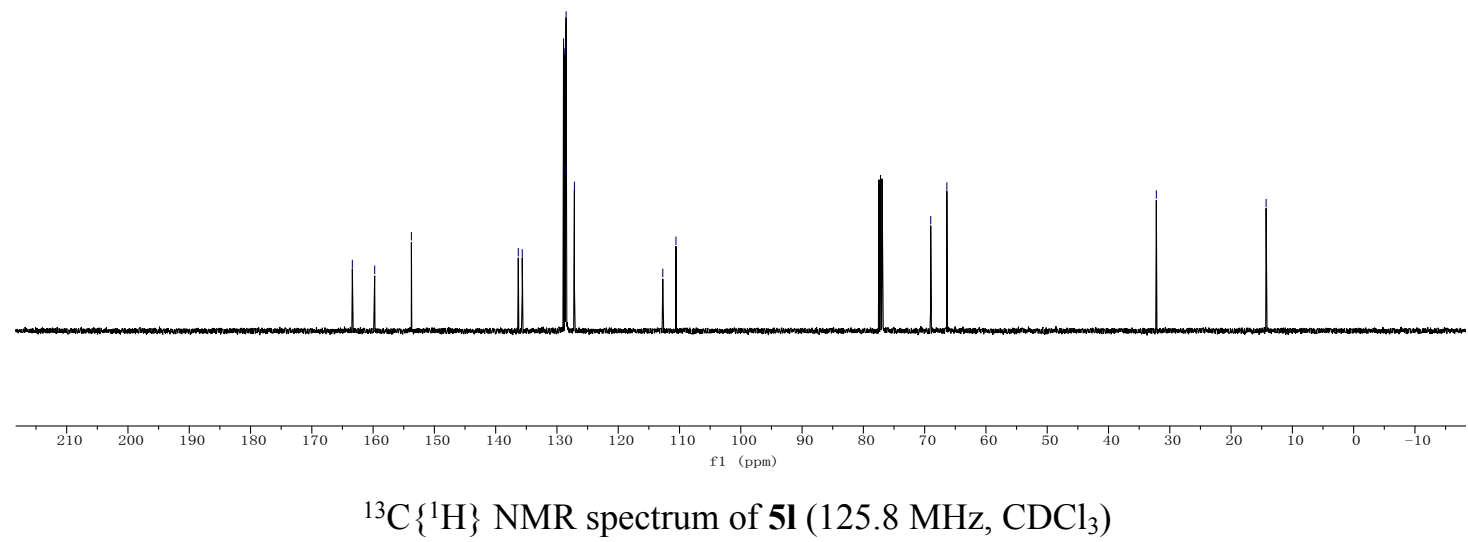


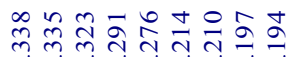

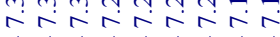

$\begin{array}{ll}n & \tilde{\delta} \\ \stackrel{n}{n} & \dot{+} \\ i & 1\end{array}$

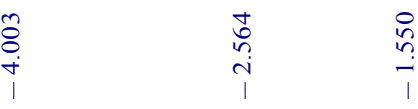
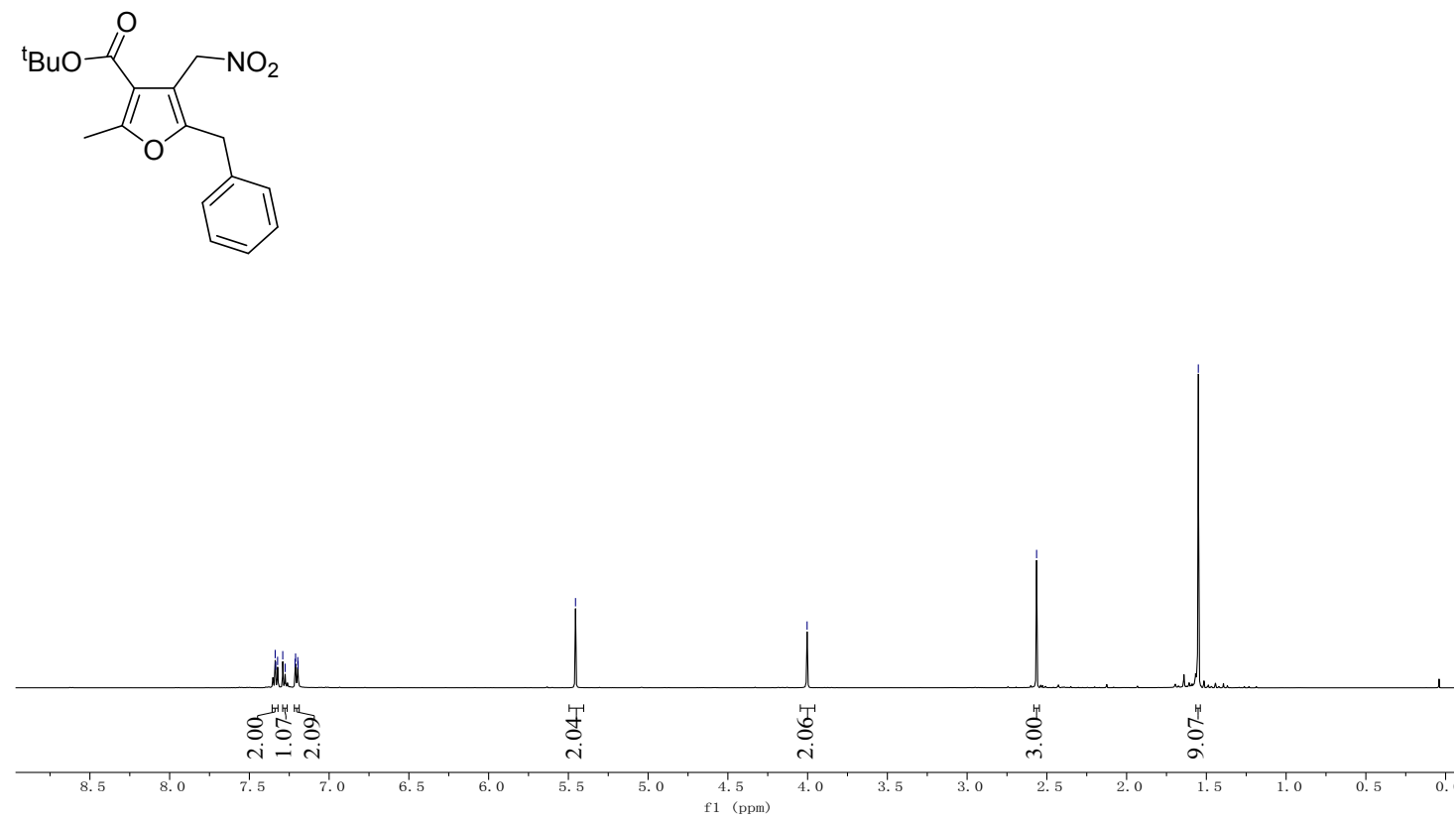

${ }^{1} \mathrm{H}$ NMR spectrum of $\mathbf{5 m}\left(500 \mathrm{MHz}, \mathrm{CDCl}_{3}\right)$

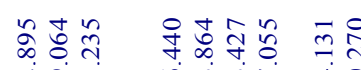

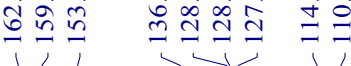

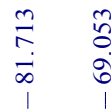

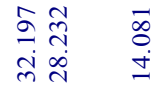

11
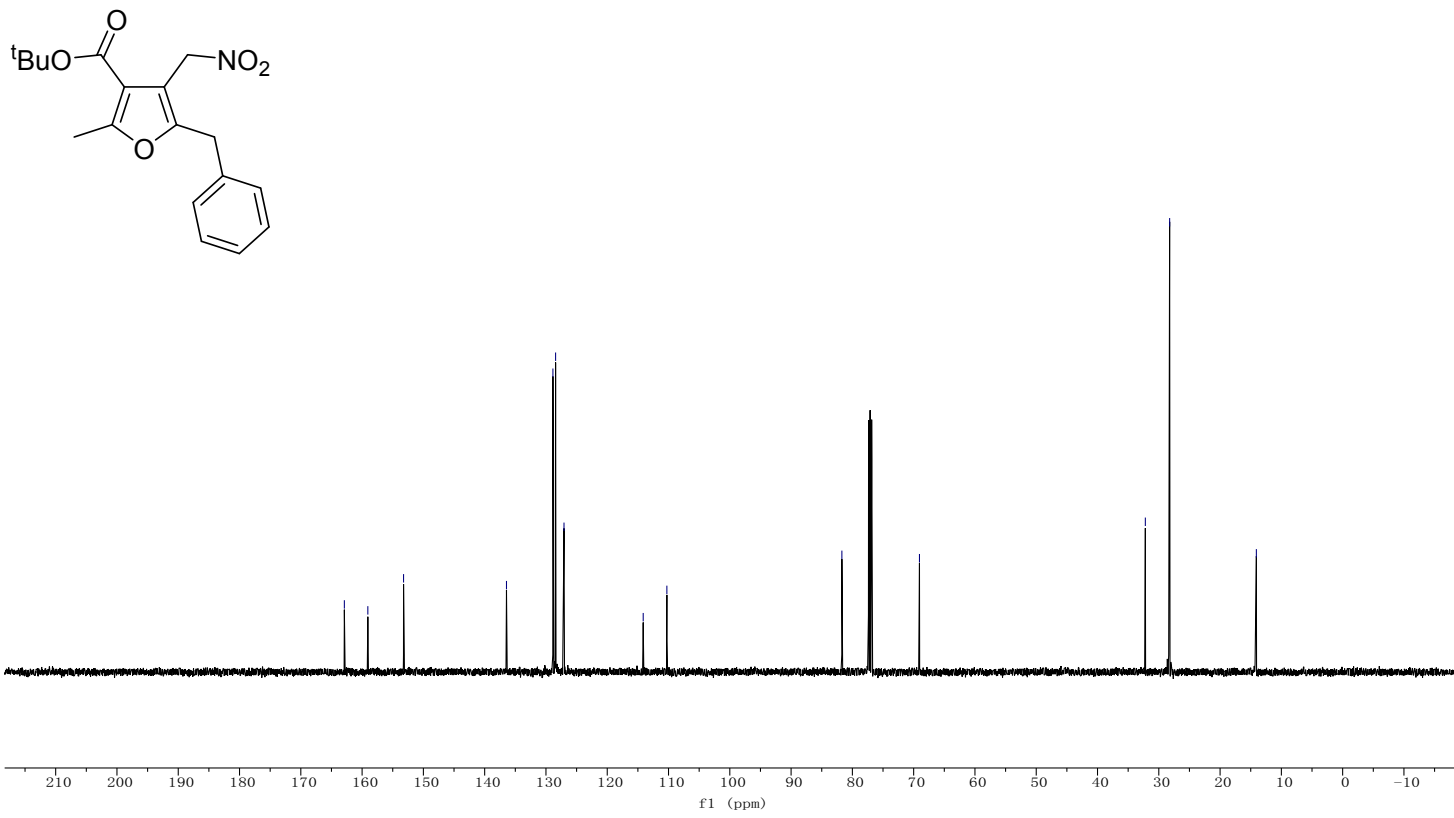

${ }^{13} \mathrm{C}\left\{{ }^{1} \mathrm{H}\right\}$ NMR spectrum of $\mathbf{5 m}\left(125.8 \mathrm{MHz}, \mathrm{CDCl}_{3}\right)$ 

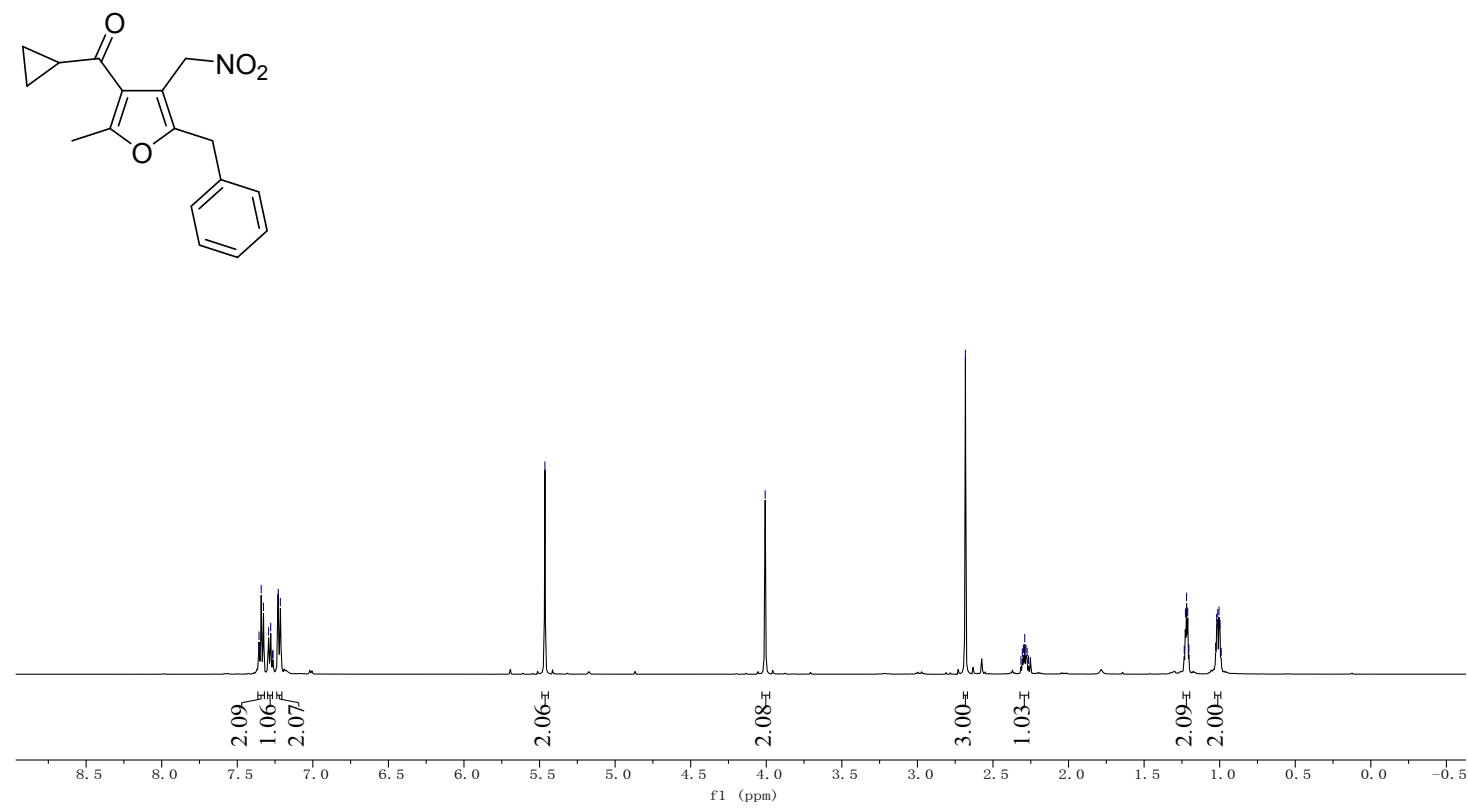

${ }^{1} \mathrm{H}$ NMR spectrum of $\mathbf{5 n}\left(500 \mathrm{MHz}, \mathrm{CDCl}_{3}\right)$

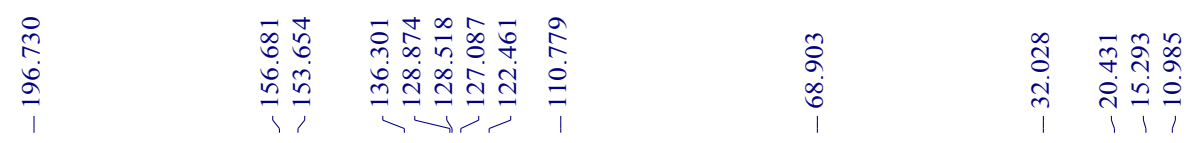<smiles>Cc1oc(Cc2ccccc2)c(C[N+](=O)[O-])c1C(=O)C1CC1</smiles>
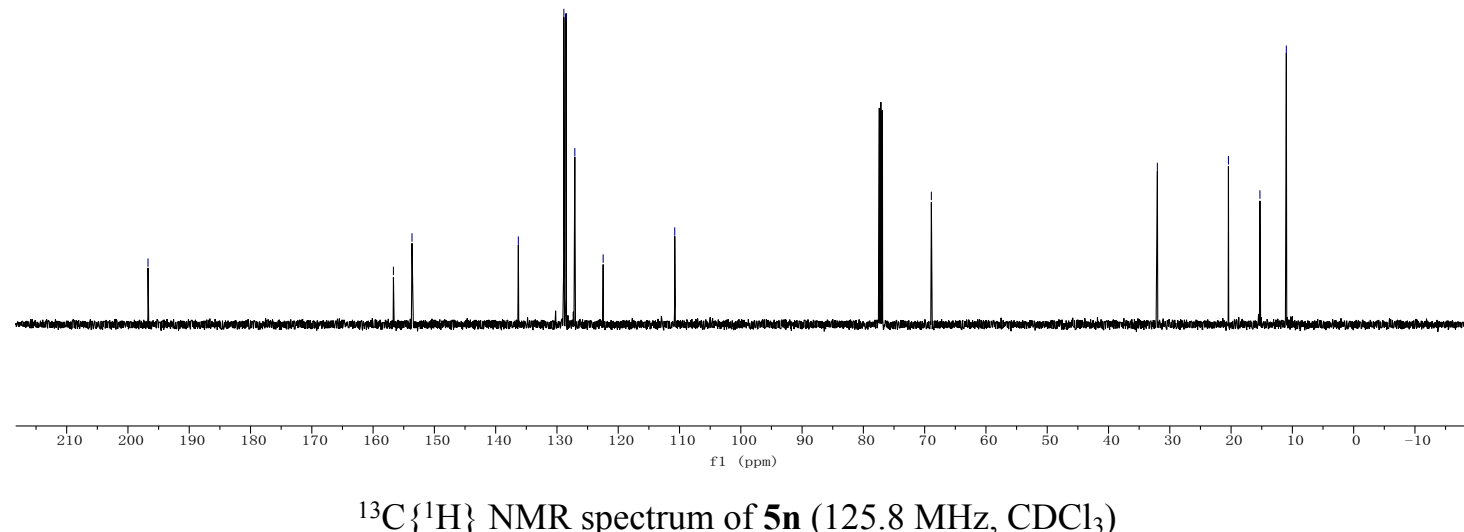

${ }^{13} \mathrm{C}\left\{{ }^{1} \mathrm{H}\right\}$ NMR spectrum of $\mathbf{5 n}\left(125.8 \mathrm{MHz}, \mathrm{CDCl}_{3}\right)$ 
<smiles>Cc1oc(Cc2ccccc2)c(C[N+](=O)[O-])c1S</smiles>

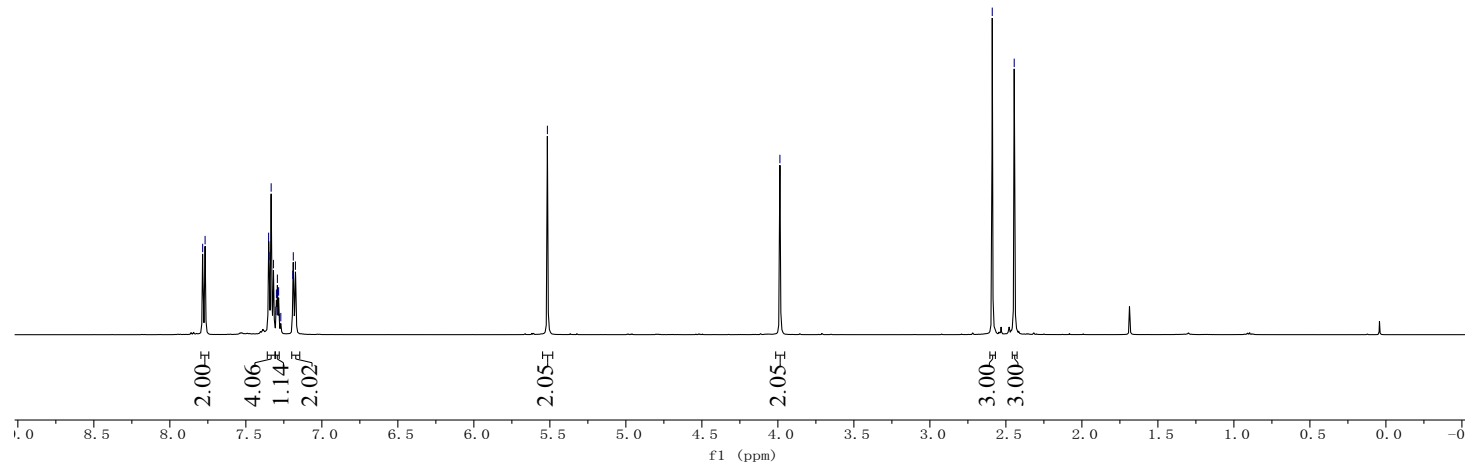

${ }^{1} \mathrm{H}$ NMR spectrum of $50\left(500 \mathrm{MHz}, \mathrm{CDCl}_{3}\right)$<smiles>Cc1oc(Cc2ccccc2)c(C[N+](=O)[O-])c1S</smiles>
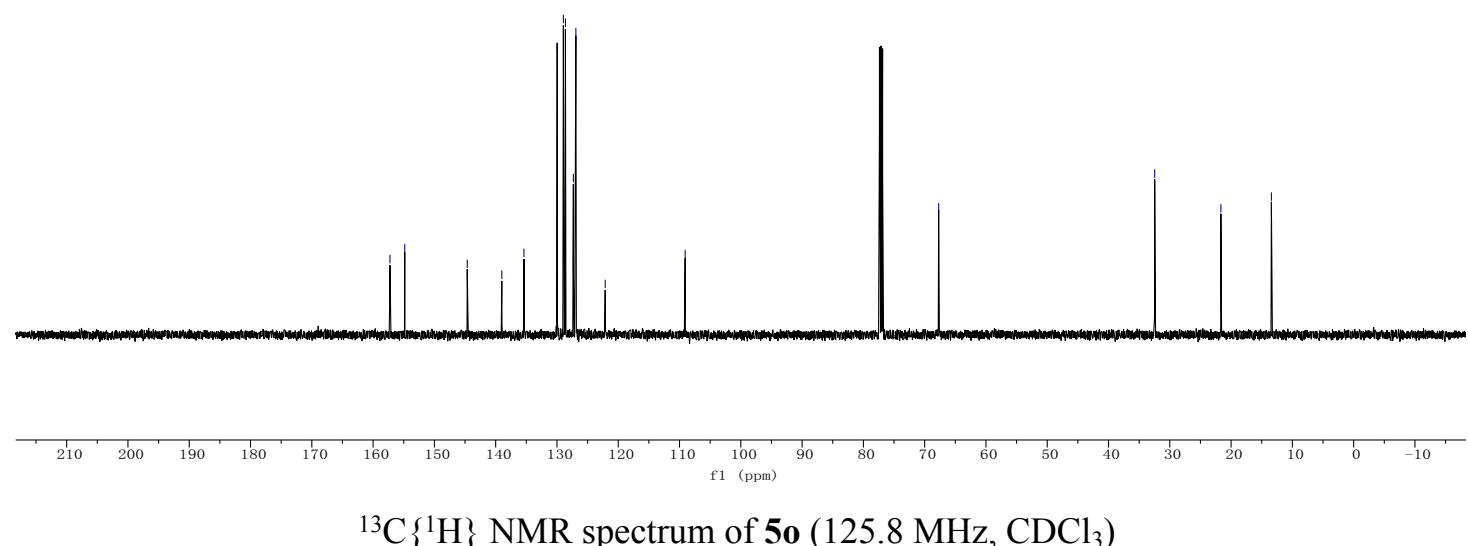

${ }^{13} \mathrm{C}\left\{{ }^{1} \mathrm{H}\right\}$ NMR spectrum of $50\left(125.8 \mathrm{MHz}, \mathrm{CDCl}_{3}\right)$ 

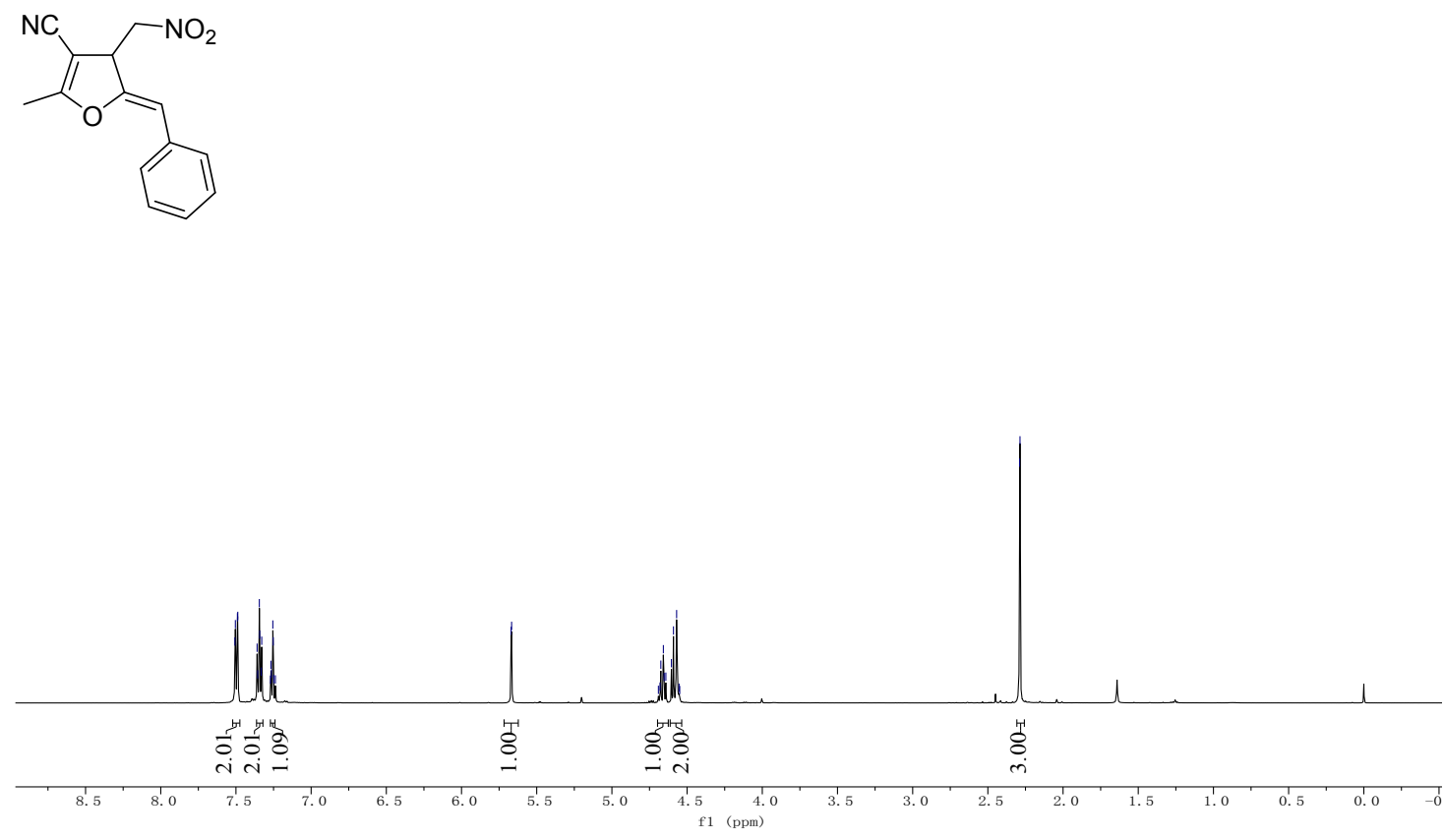

${ }^{1} \mathrm{H}$ NMR spectrum of $4 \mathrm{q}\left(500 \mathrm{MHz}, \mathrm{CDCl}_{3}\right)$

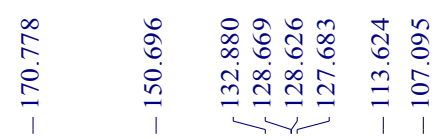<smiles>CC1=C(C#N)C(C[N+](=O)[O-])C(=Cc2ccccc2)O1</smiles>
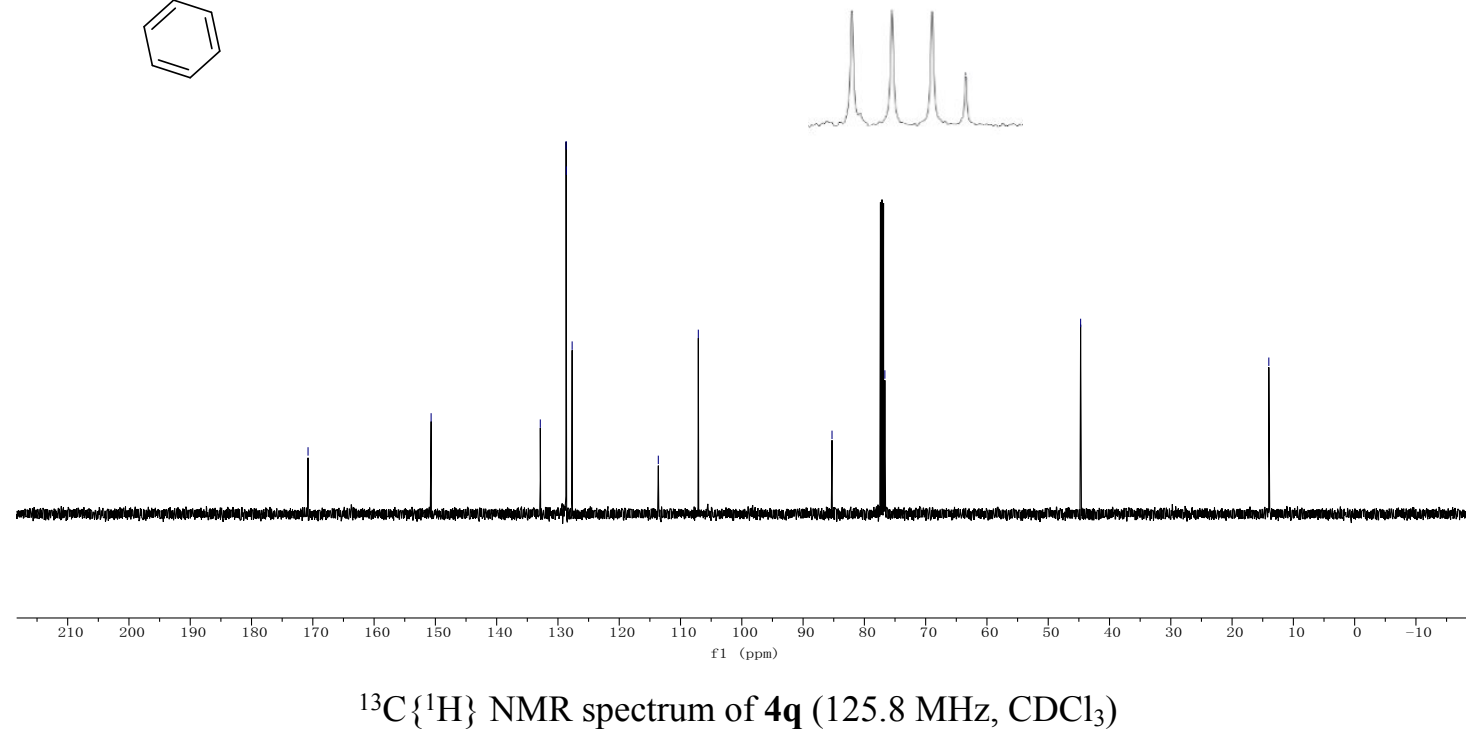
<smiles>O=C(c1ccccc1)c1c(-c2ccccc2)oc(Cc2ccccc2)c1C[N+](=O)[O-]</smiles>

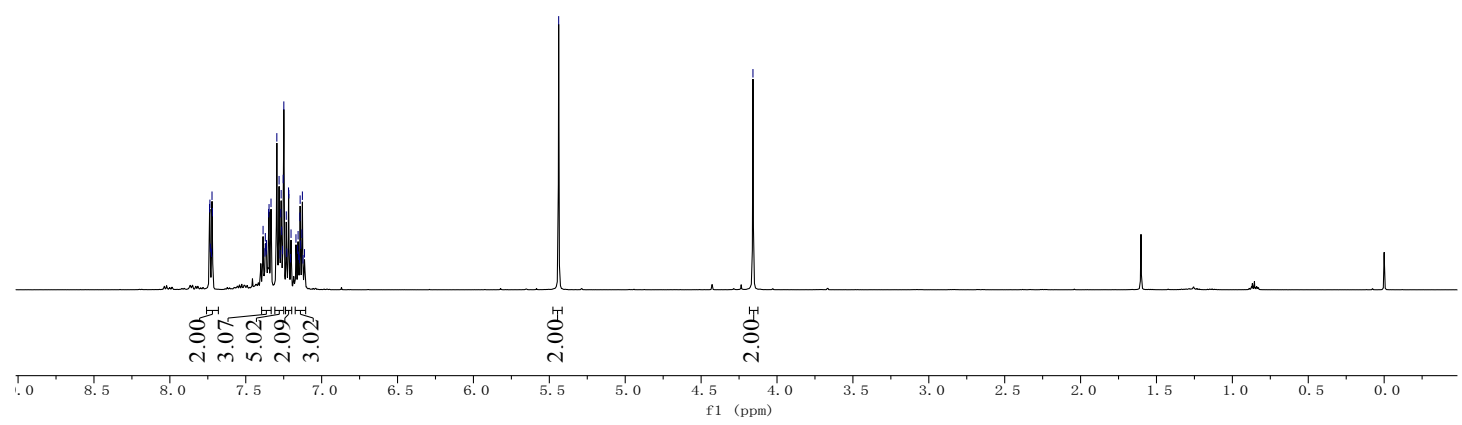

${ }^{1} \mathrm{H}$ NMR spectrum of $\mathbf{5 s}\left(500 \mathrm{MHz}, \mathrm{CDCl}_{3}\right)$

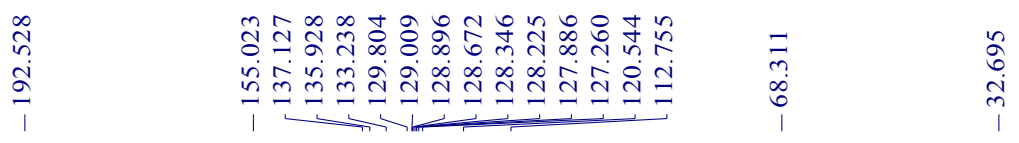<smiles>O=C(c1ccccc1)c1c(-c2ccccc2)oc(Cc2ccccc2)c1C[N+](=O)[O-]</smiles>
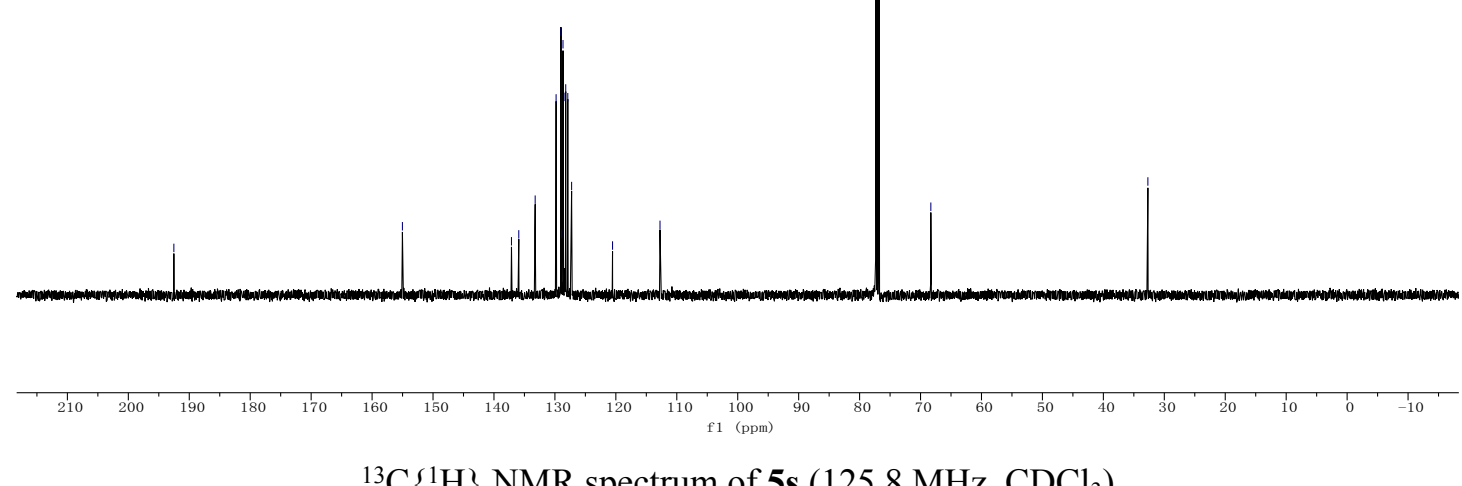

${ }^{13} \mathrm{C}\left\{{ }^{1} \mathrm{H}\right\}$ NMR spectrum of $\mathbf{5 s}\left(125.8 \mathrm{MHz}, \mathrm{CDCl}_{3}\right)$ 
<smiles>CCC(=O)c1c(CC)oc(Cc2ccccc2)c1C[N+](=O)[O-]</smiles>

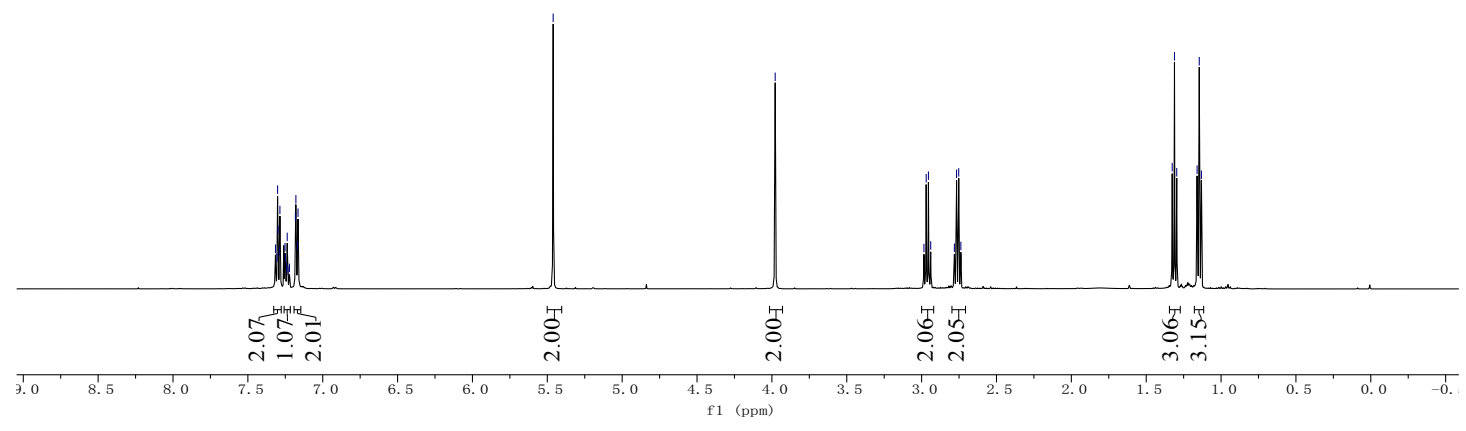

${ }^{1} \mathrm{H}$ NMR spectrum of $\mathbf{5 t}\left(500 \mathrm{MHz}, \mathrm{CDCl}_{3}\right)$

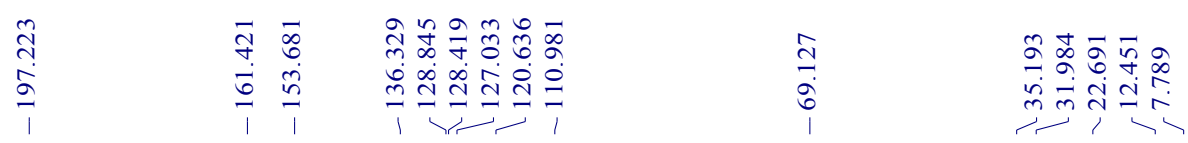<smiles>CCC(=O)c1c(CC)oc(Cc2ccccc2)c1C[N+](=O)[O-]</smiles>
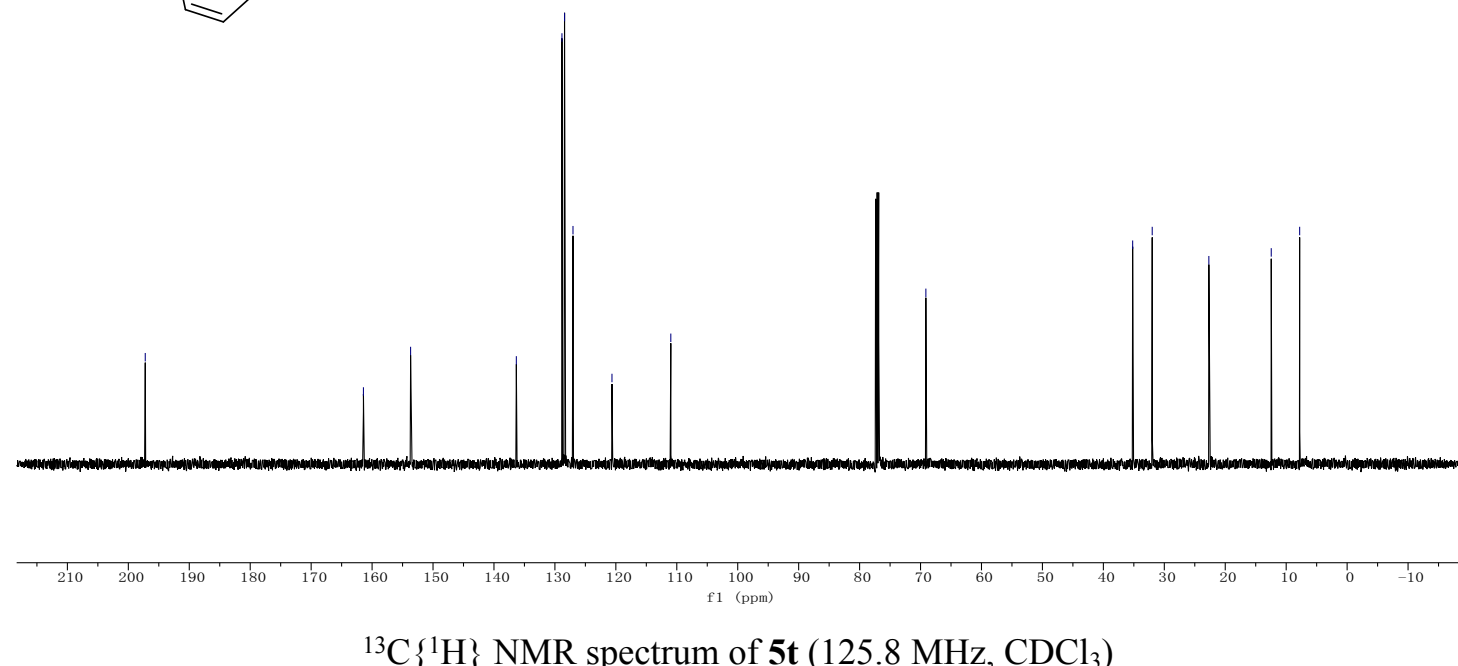

${ }^{13} \mathrm{C}\left\{{ }^{1} \mathrm{H}\right\}$ NMR spectrum of $\mathbf{5 t}\left(125.8 \mathrm{MHz}, \mathrm{CDCl}_{3}\right)$ 


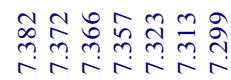

$\begin{array}{ll}\vec{m} & \overrightarrow{0} \\ \overrightarrow{7} & 0 \\ + & 0 \\ 1 & 1\end{array}$

(1)

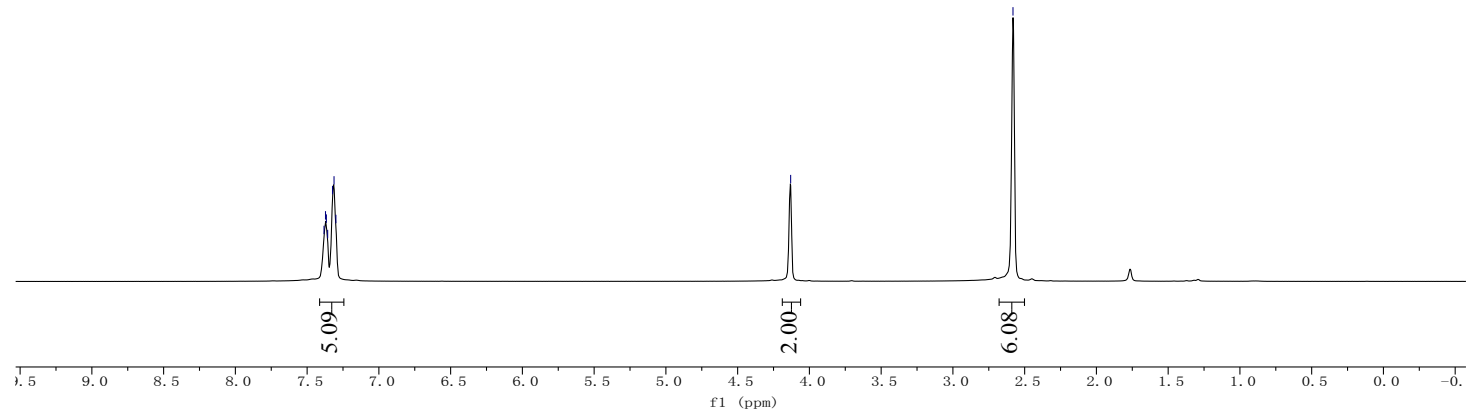

${ }^{1} \mathrm{H}$ NMR spectrum of $7 \mathbf{a}\left(500 \mathrm{MHz}, \mathrm{CDCl}_{3}\right)$

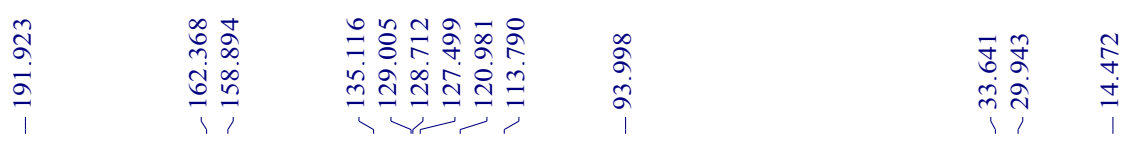<smiles>CC(=O)c1c(C)oc(Cc2ccccc2)c1C#N</smiles>
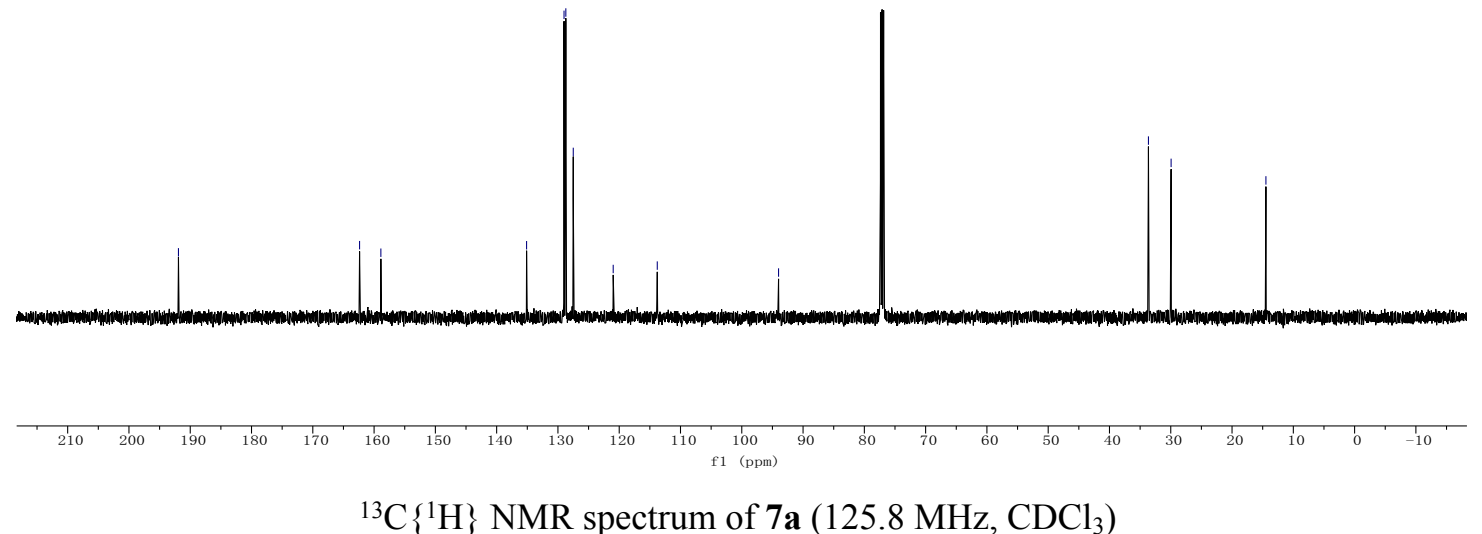

${ }^{13} \mathrm{C}\left\{{ }^{1} \mathrm{H}\right\}$ NMR spectrum of $7 \mathbf{a}\left(125.8 \mathrm{MHz}, \mathrm{CDCl}_{3}\right)$ 

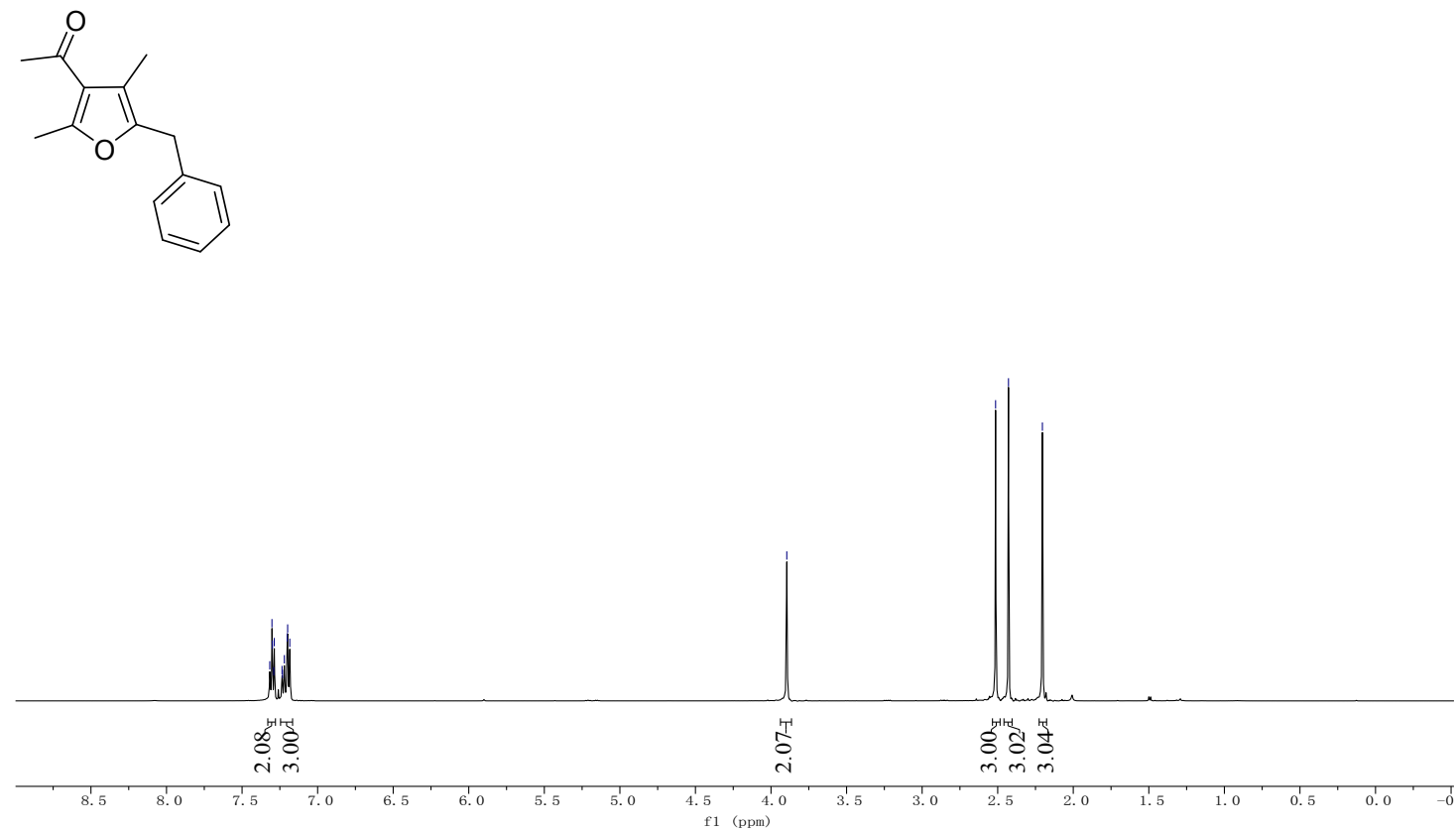

${ }^{1} \mathrm{H}$ NMR spectrum of $\mathbf{8 a}\left(500 \mathrm{MHz}, \mathrm{CDCl}_{3}\right)$

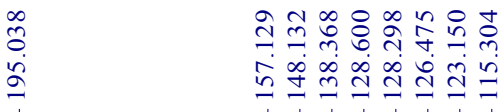

(1)
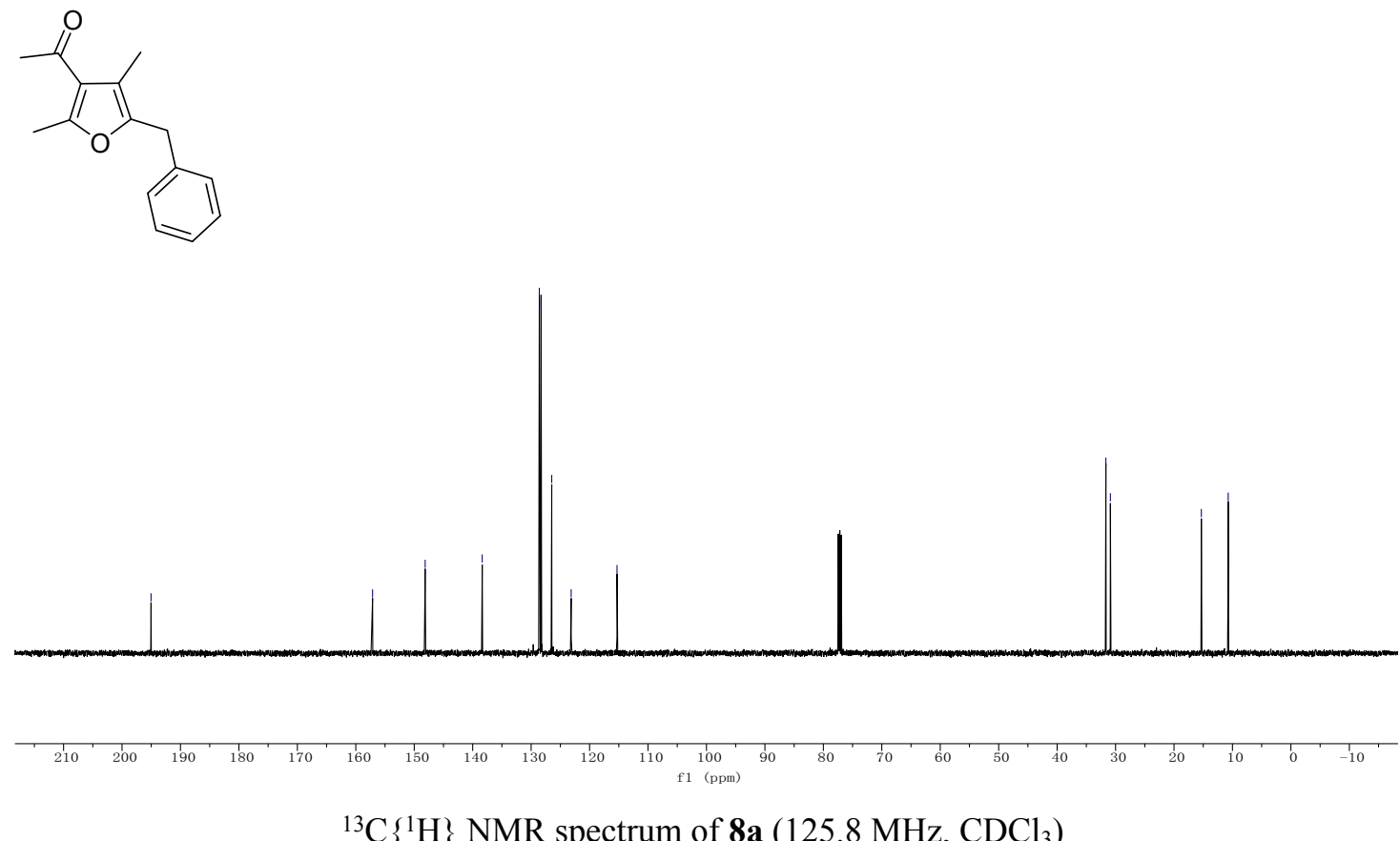

${ }^{13} \mathrm{C}\left\{{ }^{1} \mathrm{H}\right\}$ NMR spectrum of $\mathbf{8 a}\left(125.8 \mathrm{MHz}, \mathrm{CDCl}_{3}\right)$ 

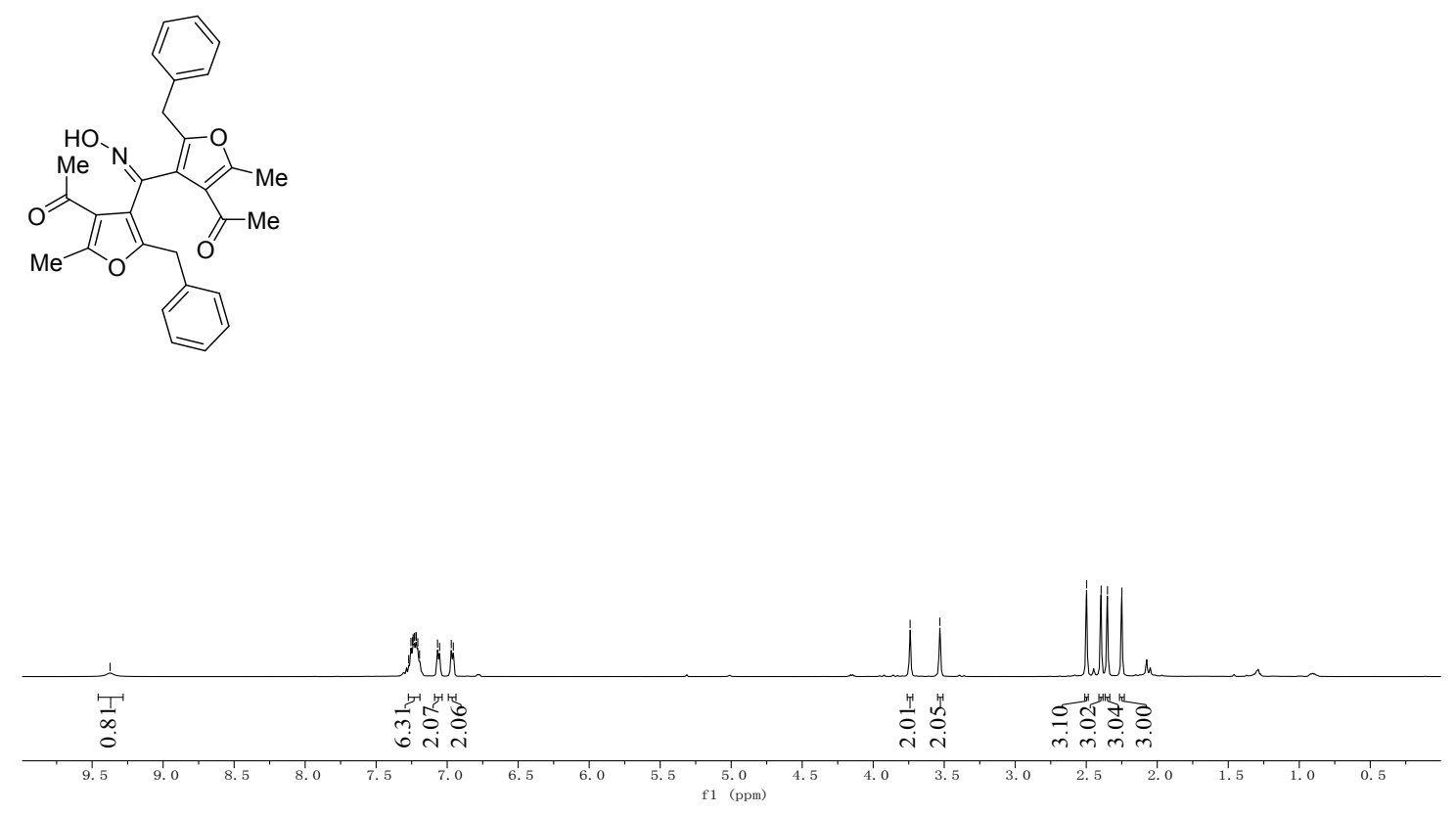

${ }^{1} \mathrm{H}$ NMR spectrum of $9 \mathbf{a}\left(500 \mathrm{MHz}, \mathrm{CDCl}_{3}\right)$<smiles>CC(=O)c1c(C)oc(Cc2ccccc2)c1/C(=N/O)c1c(Cc2ccccc2)oc(C)c1C(=O)O</smiles>

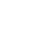


<smiles>CC(=O)c1c(C)oc(Cc2ccc(C)cc2)c1C(=O)c1c(Cc2ccc(C)cc2)oc(C)c1C(N)=O</smiles>

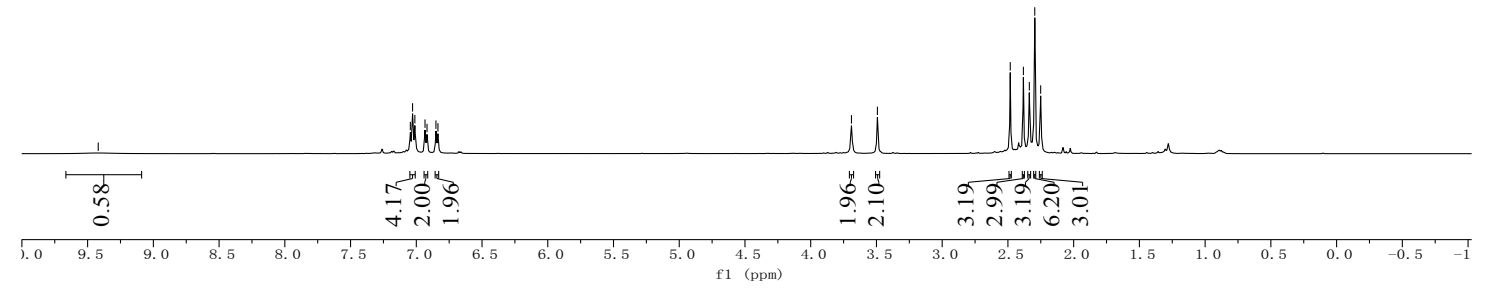

${ }^{1} \mathrm{H}$ NMR spectrum of $\mathbf{9 b}\left(500 \mathrm{MHz}, \mathrm{CDCl}_{3}\right)$

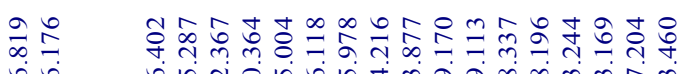

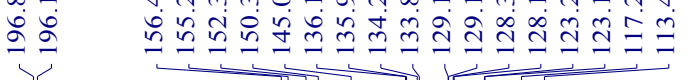

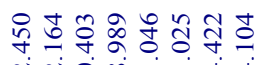

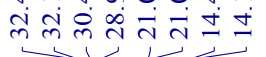<smiles>CC(=O)c1c(C)oc(Cc2ccc(C)cc2)c1/C(=N\O)c1c(Cc2ccc(C)cc2)oc(C)c1C(=O)O</smiles>
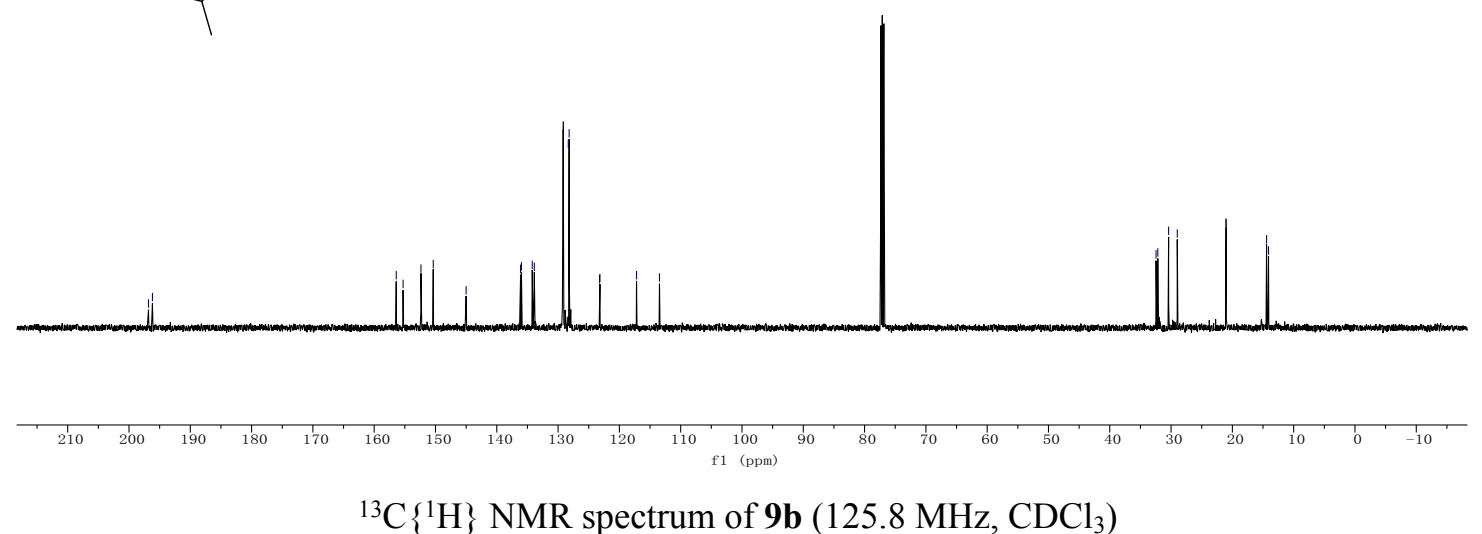

${ }^{13} \mathrm{C}\left\{{ }^{1} \mathrm{H}\right\}$ NMR spectrum of $\mathbf{9 b}\left(125.8 \mathrm{MHz}, \mathrm{CDCl}_{3}\right)$ 
<smiles>CC(=O)c1c(C)oc(Cc2ccc(C(C)C)cc2)c1C(=NO)c1c(Cc2ccc(C(C)C)cc2)oc(C)c1C(C)=O</smiles>

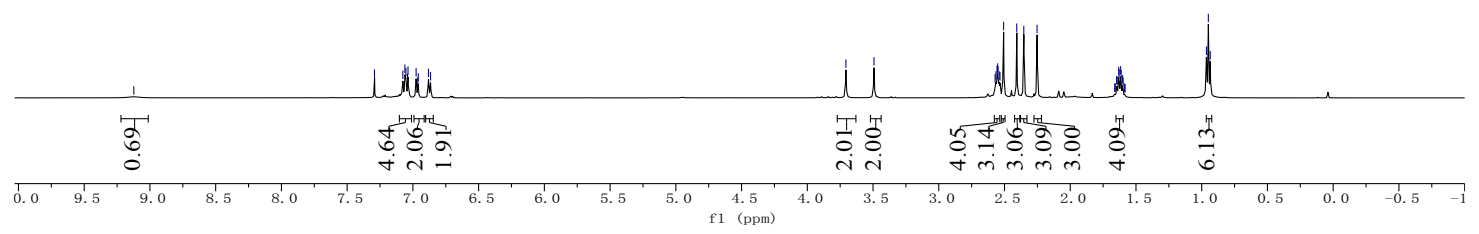

${ }^{1} \mathrm{H}$ NMR spectrum of 9c $\left(500 \mathrm{MHz}, \mathrm{CDCl}_{3}\right)$

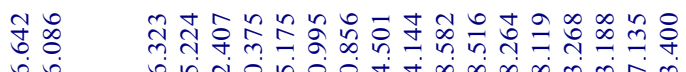

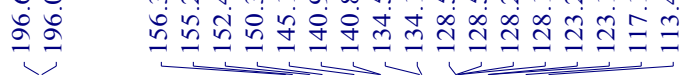

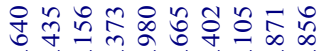

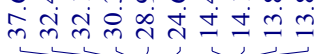
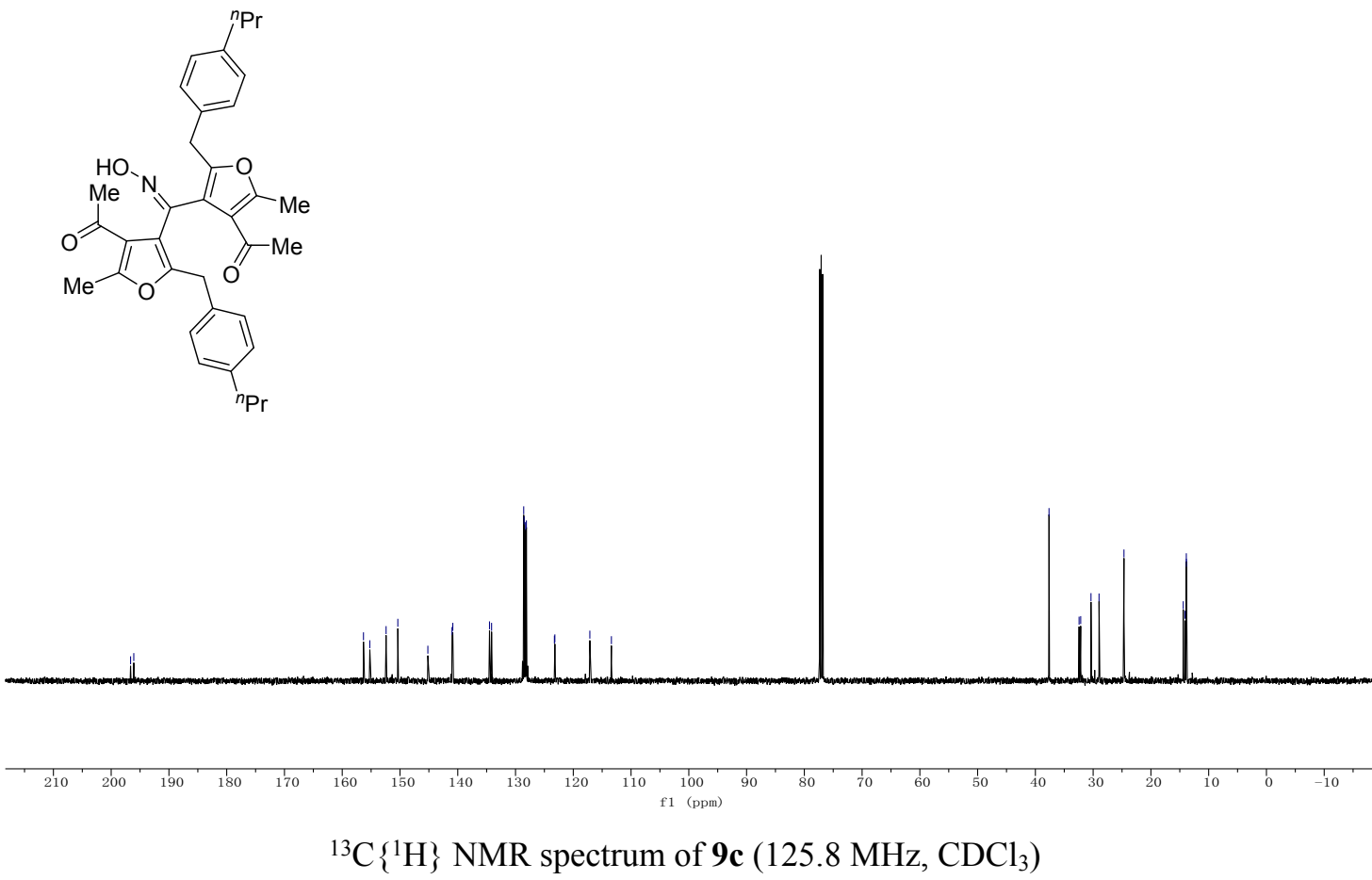

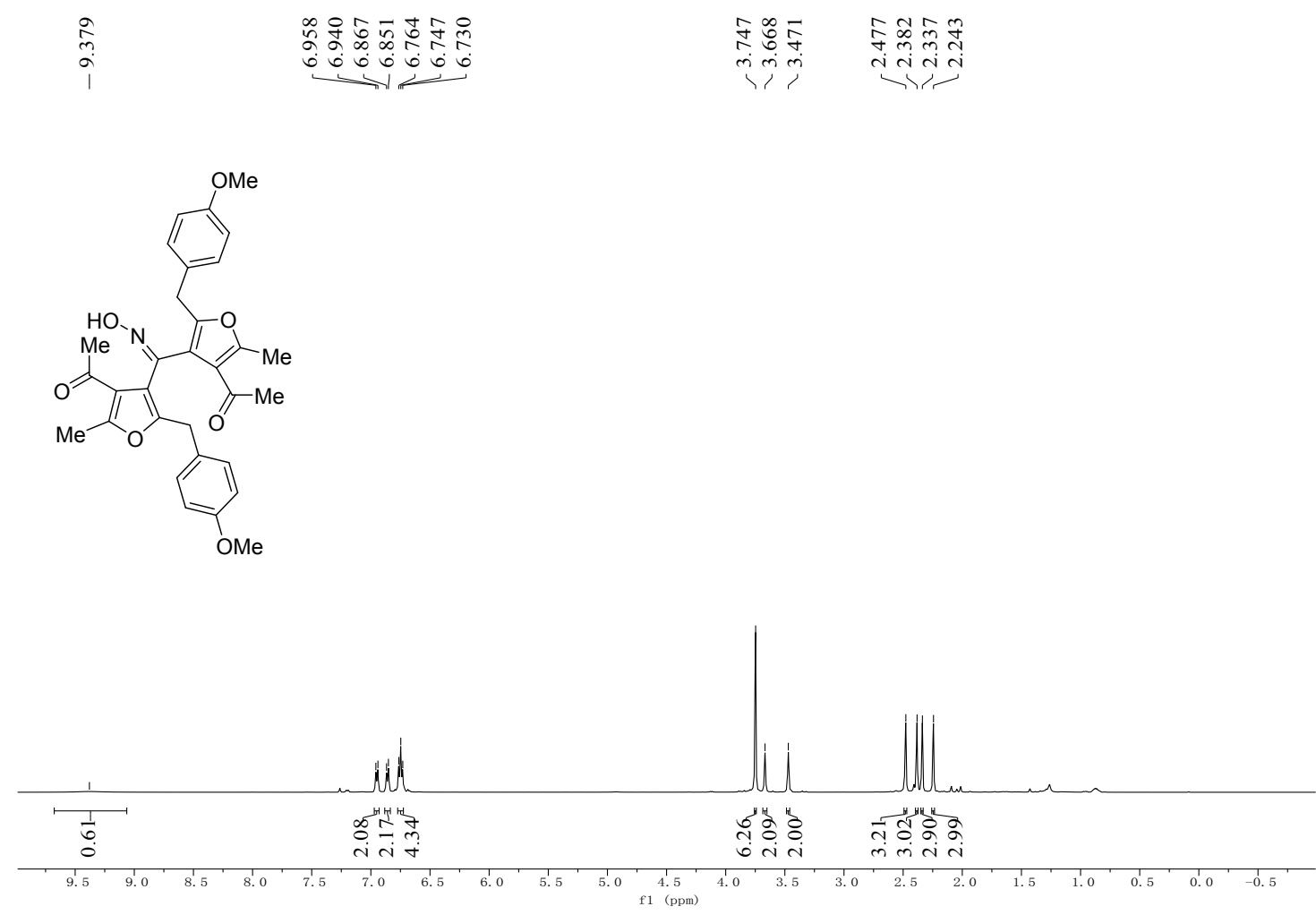

${ }^{1} \mathrm{H}$ NMR spectrum of $9 d\left(500 \mathrm{MHz}, \mathrm{CDCl}_{3}\right)$

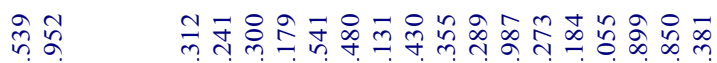

。ํ.
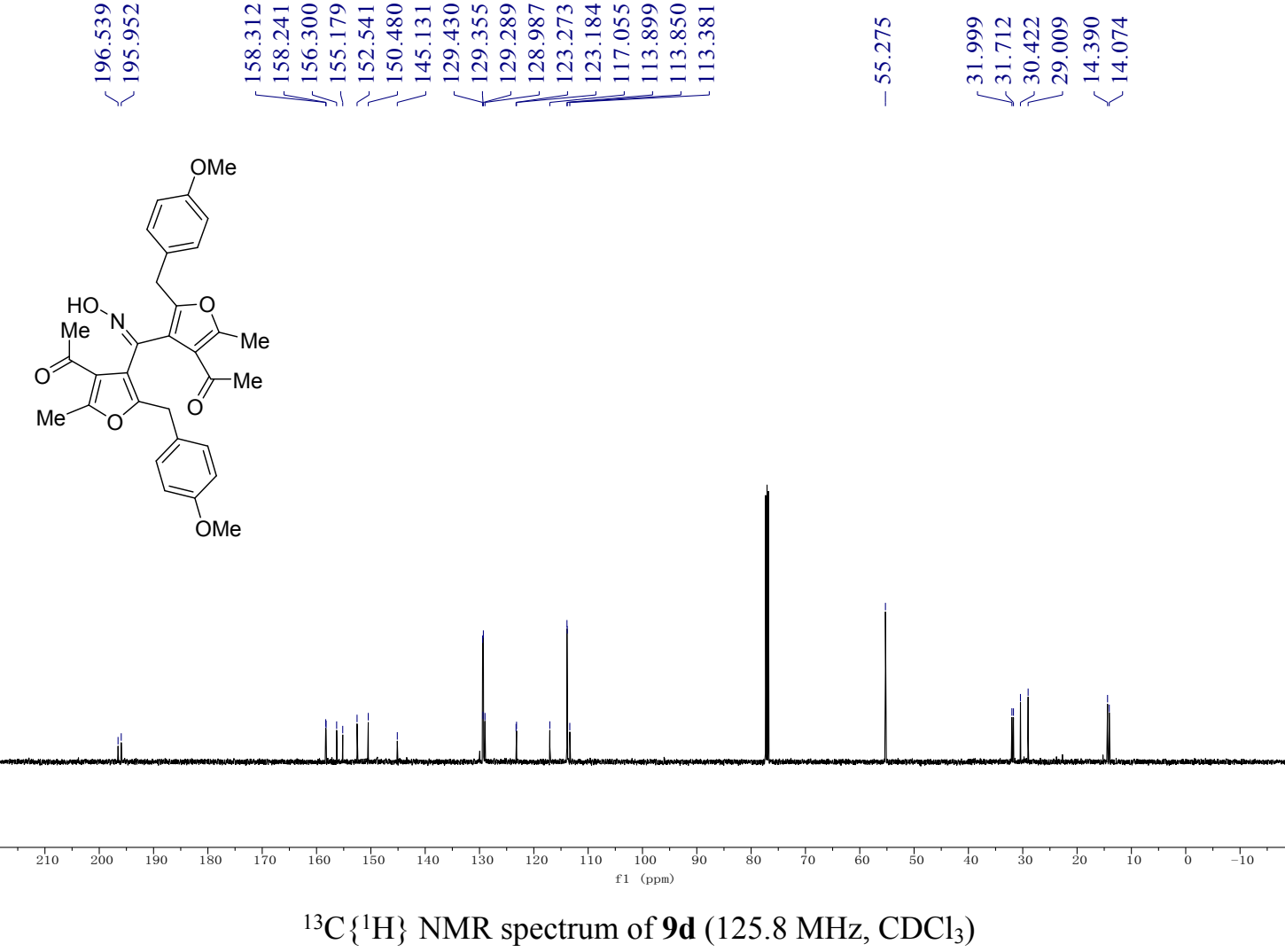


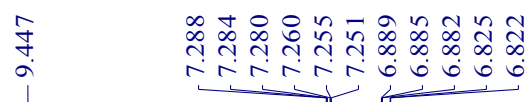

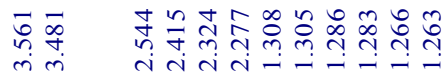<smiles>CC(=O)c1c(C)oc(Cc2cc(C(C)(C)C)cc(C(C)(C)C)c2)c1C(=NO)c1c(Cc2cc(C(C)(C)C)cc(C(C)(C)C)c2)oc(C)c1C(C)=O</smiles>

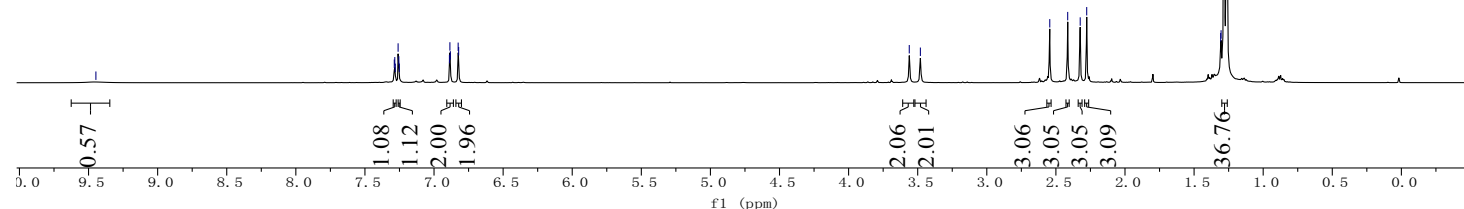

${ }^{1} \mathrm{H}$ NMR spectrum of 9e $\left(500 \mathrm{MHz}, \mathrm{CDCl}_{3}\right)$
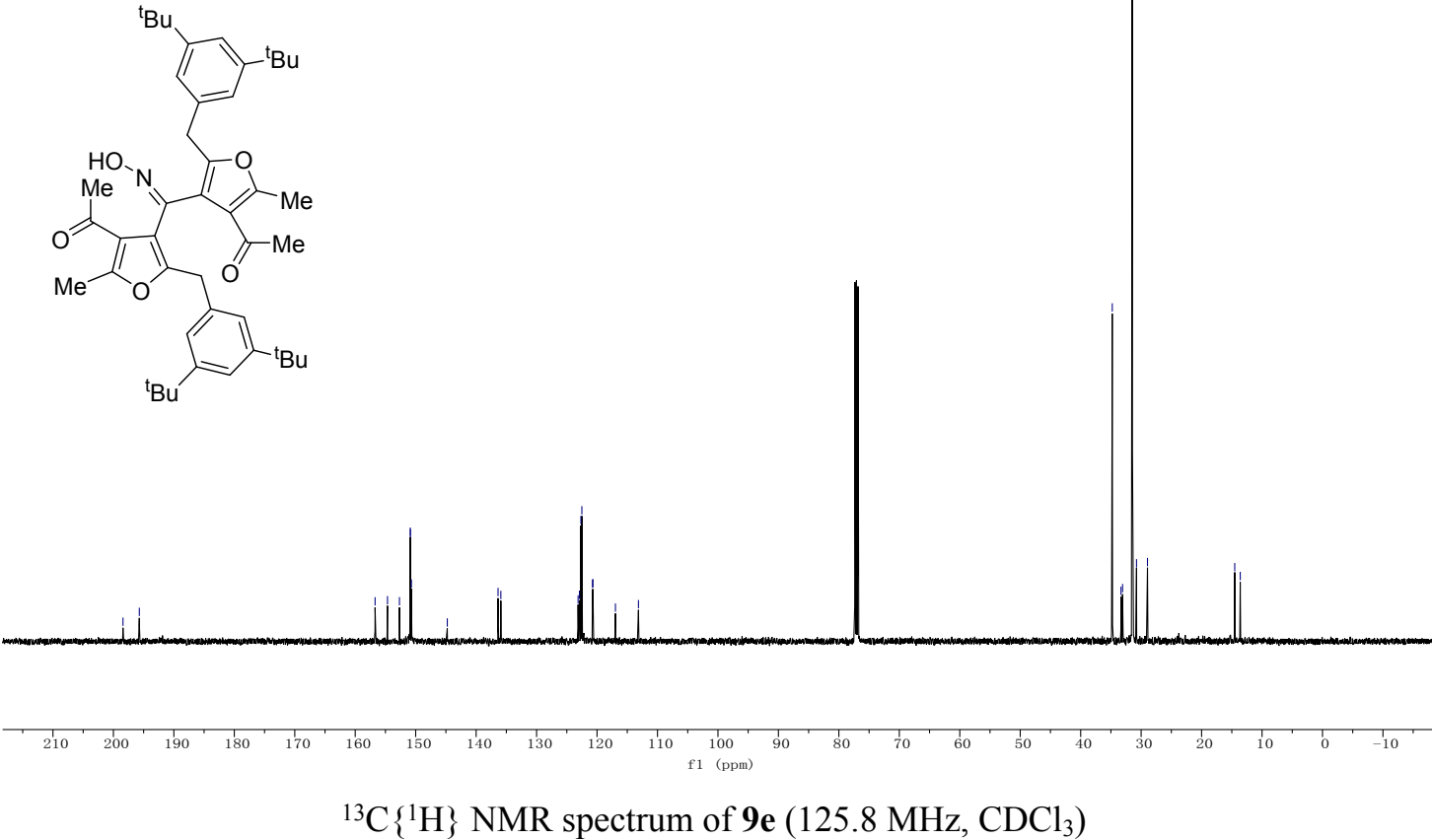
<smiles>CC(=O)c1c(C)oc(Cc2ccc(Cl)cc2)c1C(=O)c1c(C(=O)O)oc(C)c1C(=O)O</smiles>

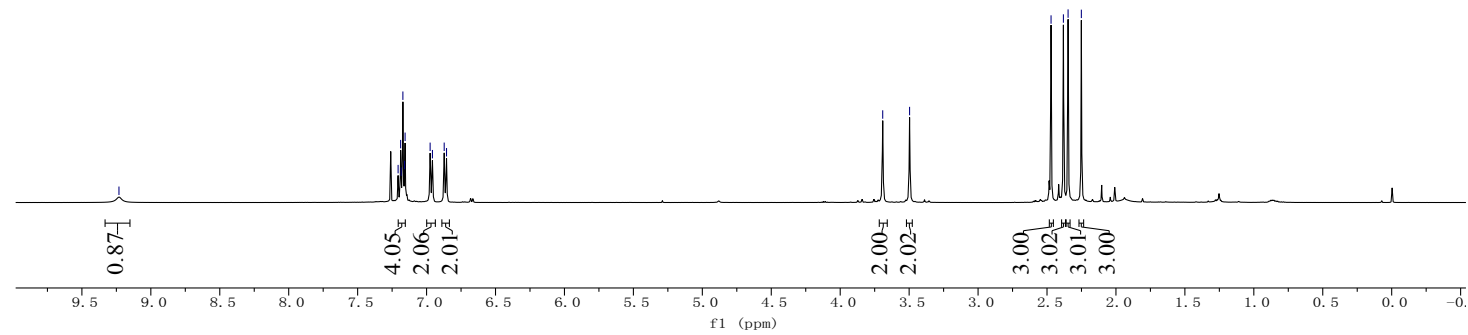

${ }^{1} \mathrm{H}$ NMR spectrum of $9 f\left(500 \mathrm{MHz}, \mathrm{CDCl}_{3}\right)$

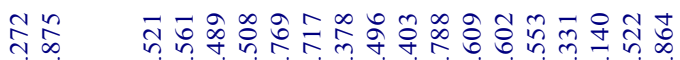

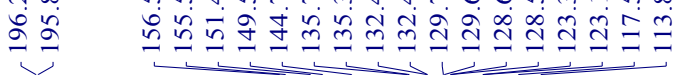

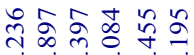
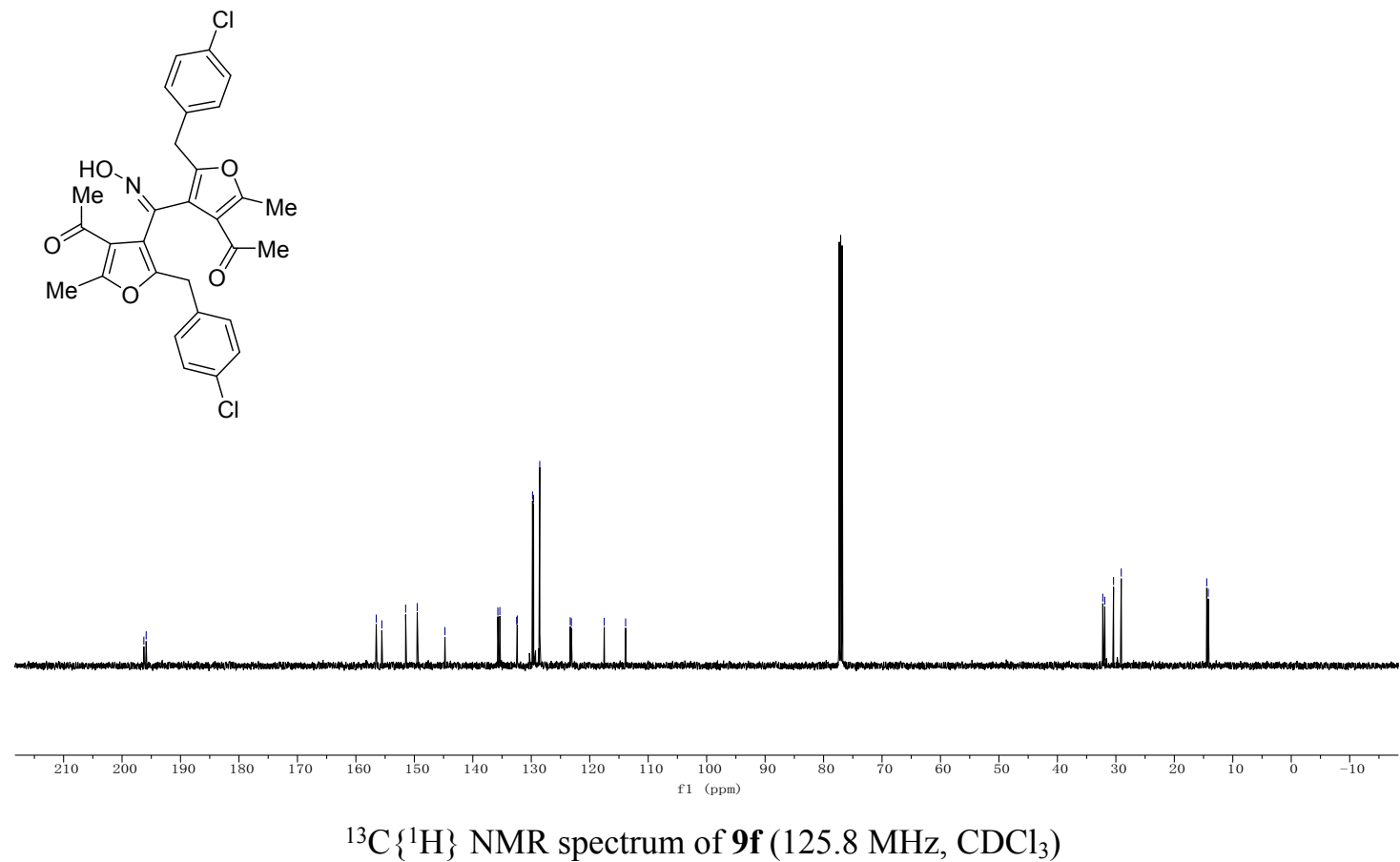
<smiles>CC(=O)c1c(C)oc(Cc2ccc(F)cc2)c1C(=NO)c1c(Cc2ccc(F)cc2)oc(C)c1C(C)=O</smiles>

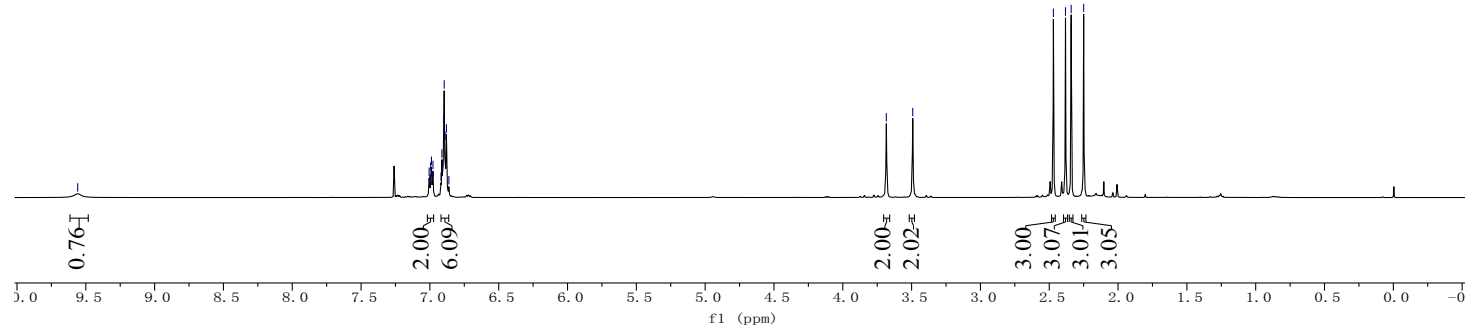

${ }^{1} \mathrm{H}$ NMR spectrum of $\mathbf{9 g}\left(500 \mathrm{MHz}, \mathrm{CDCl}_{3}\right)$

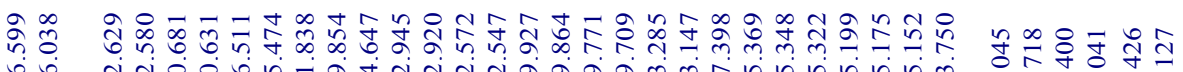

ま
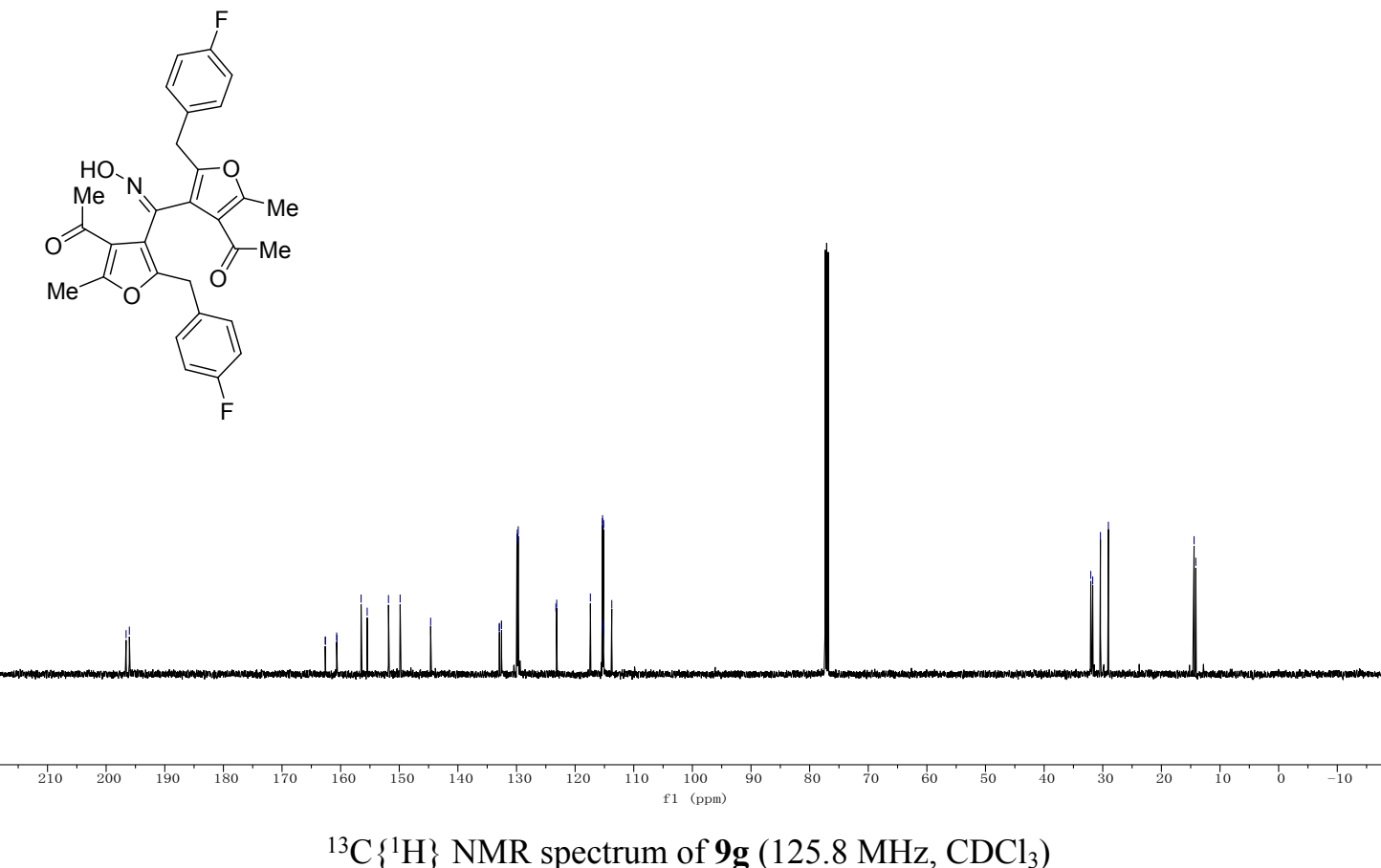

${ }^{13} \mathrm{C}\left\{{ }^{1} \mathrm{H}\right\}$ NMR spectrum of $\mathbf{9 g}\left(125.8 \mathrm{MHz}, \mathrm{CDCl}_{3}\right)$ 
<smiles>COc1c(Cc2ccccc2)oc(C)c1/C(=N/O)c1c(Cc2ccccc2)oc(C)c1C(=O)O</smiles>

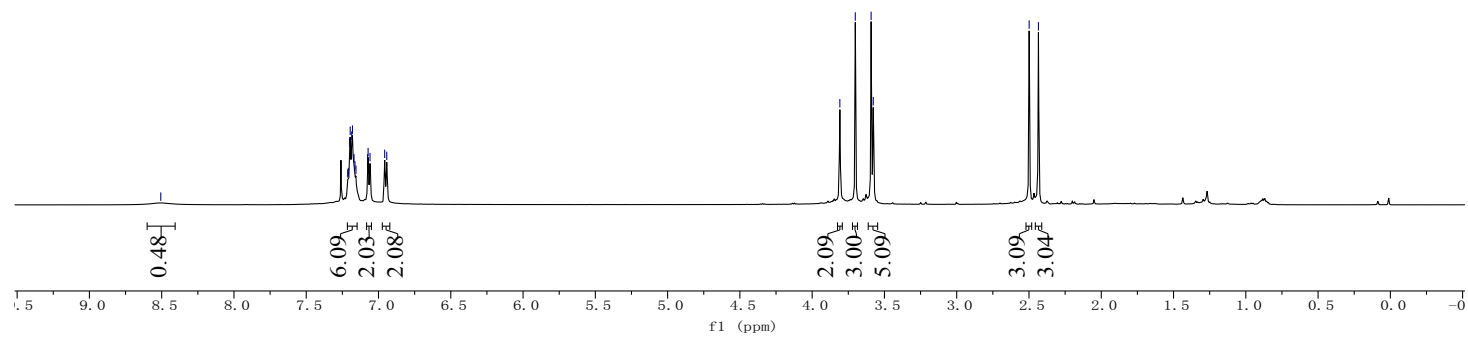

${ }^{1} \mathrm{H}$ NMR spectrum of $9 \mathbf{i}\left(500 \mathrm{MHz}, \mathrm{CDCl}_{3}\right)$<smiles>COC(=O)c1c(C)oc(Cc2ccccc2)c1C(=O)c1c(Cc2ccccc2)oc(C)c1C(=O)O</smiles>
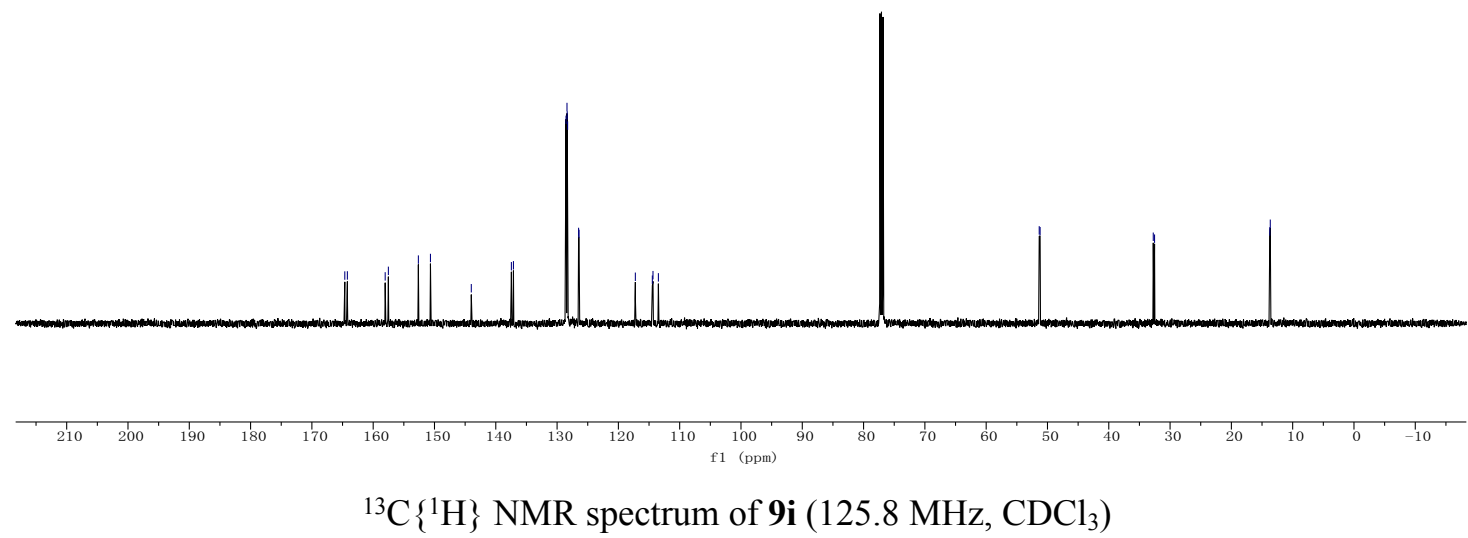

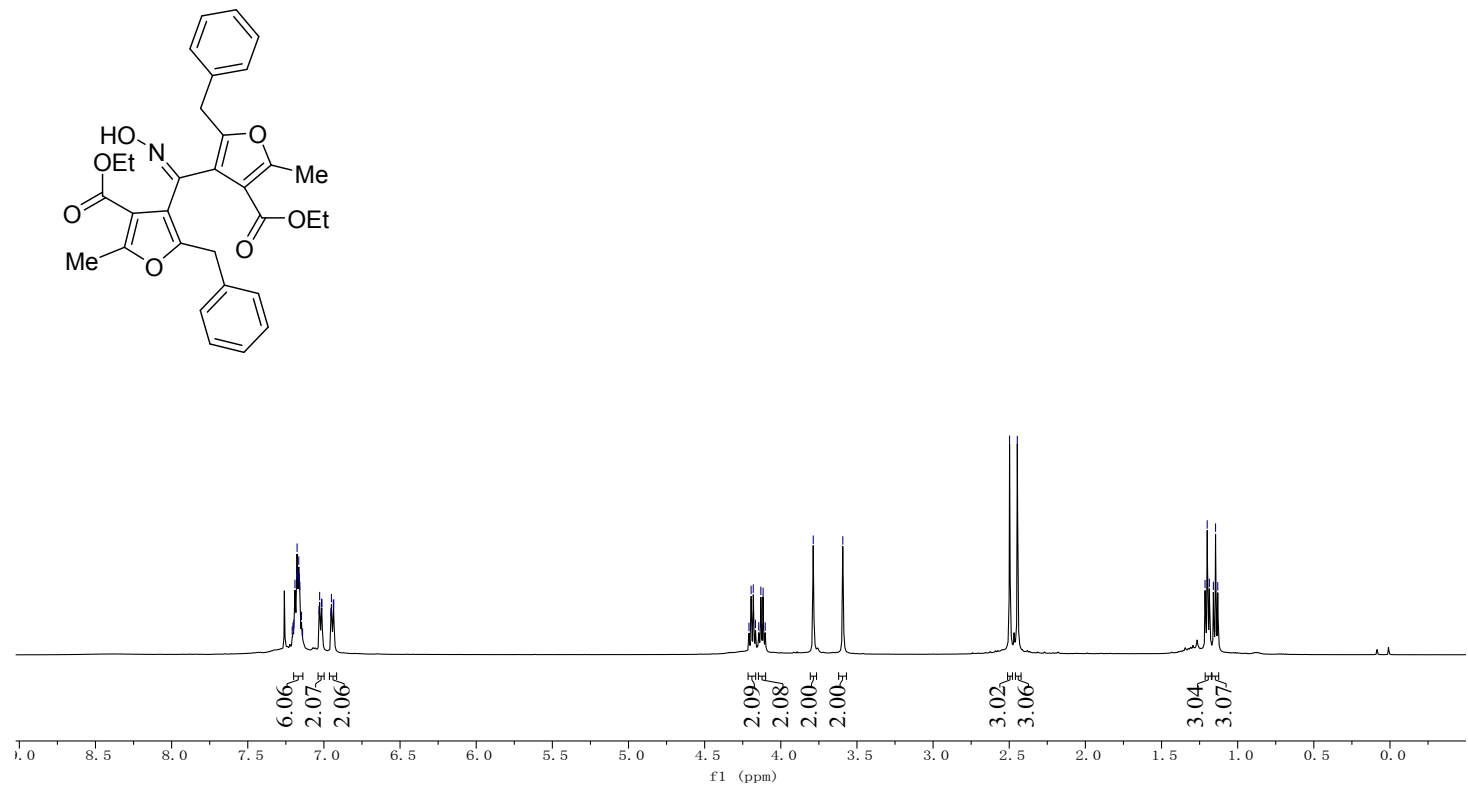

${ }^{1} \mathrm{H}$ NMR spectrum of $\mathbf{9 j}\left(500 \mathrm{MHz}, \mathrm{CDCl}_{3}\right)$
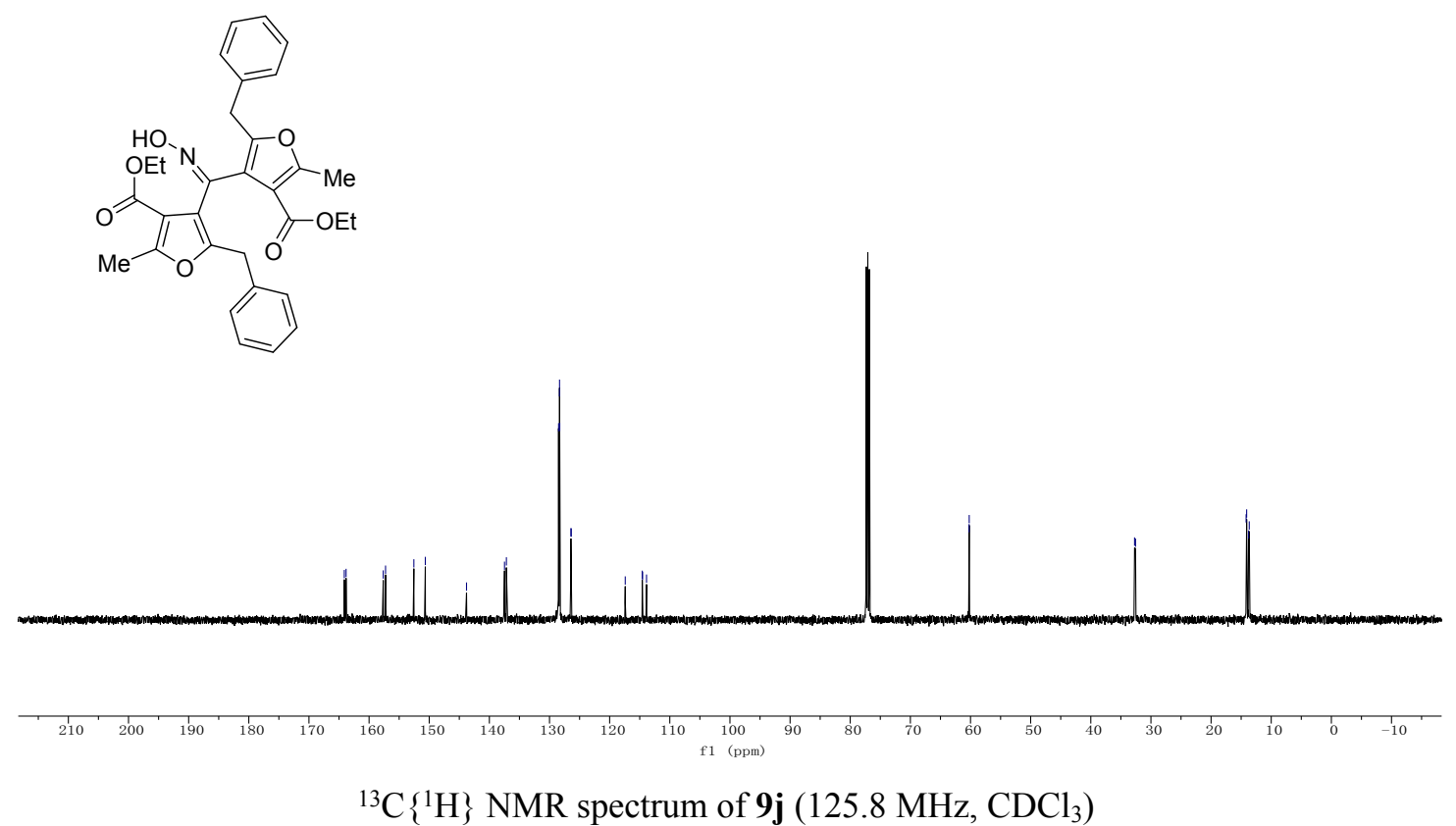
<smiles>C=CCOC(=O)c1c(C)oc(Cc2ccccc2)c1/C(=N\O)c1c(Cc2ccccc2)oc(C)c1C(=O)OCCC</smiles>

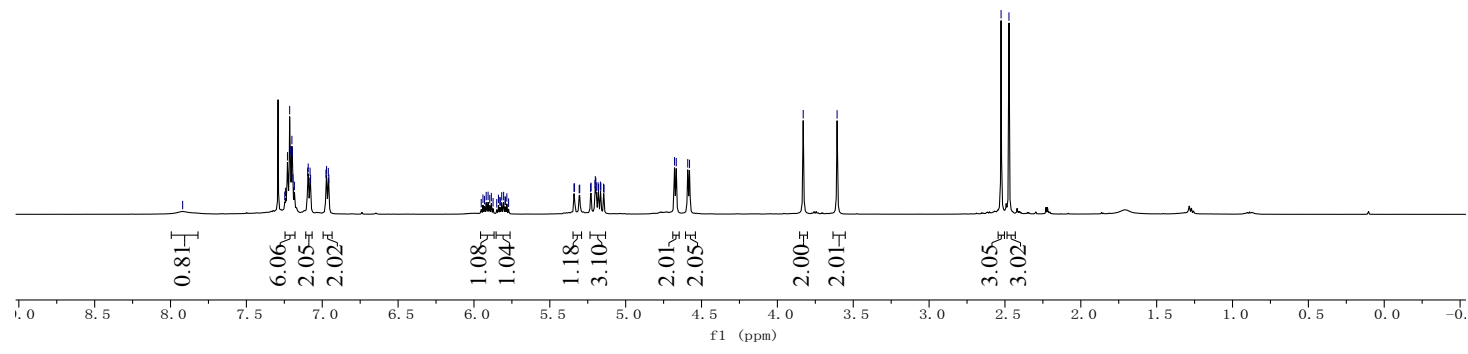

${ }^{1} \mathrm{H}$ NMR spectrum of $9 \mathbf{k}\left(500 \mathrm{MHz}, \mathrm{CDCl}_{3}\right)$

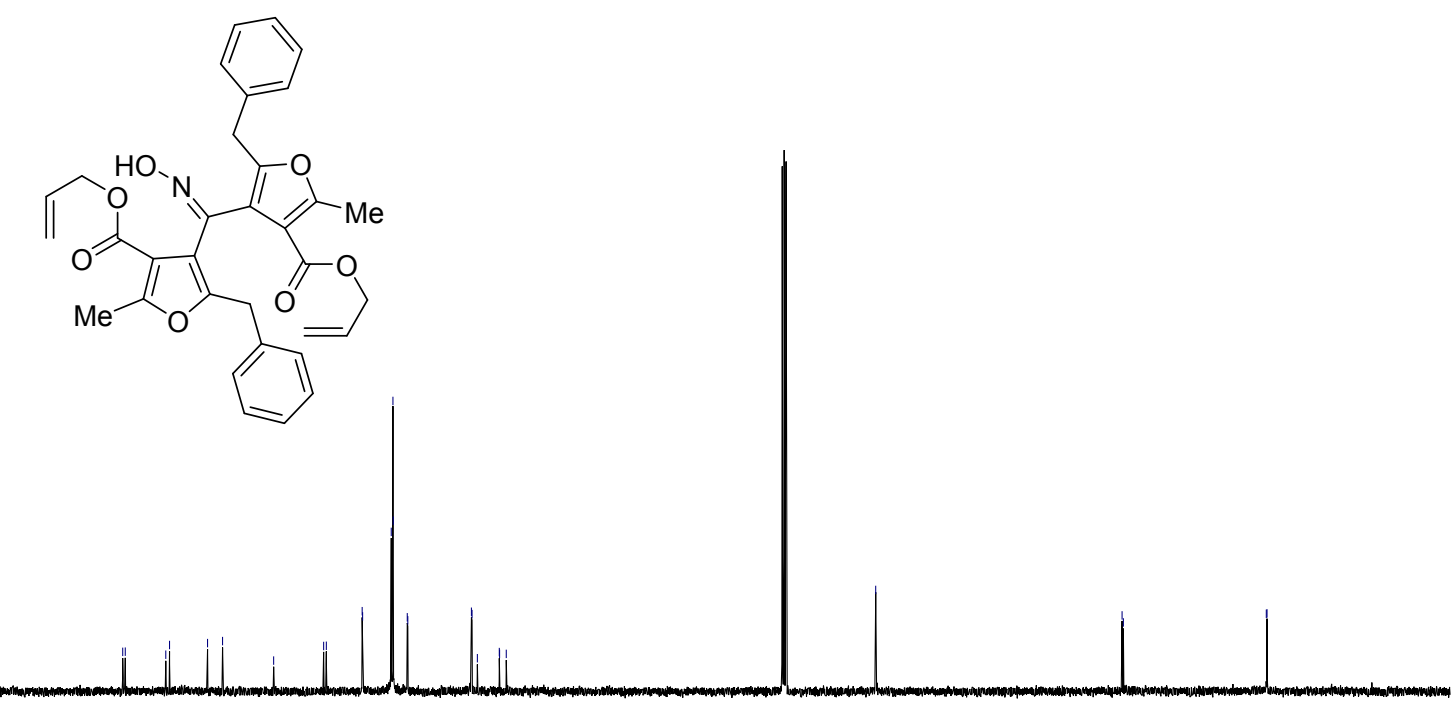

${ }^{13} \mathrm{C}\left\{{ }^{1} \mathrm{H}\right\}$ NMR spectrum of $9 \mathbf{k}\left(125.8 \mathrm{MHz}, \mathrm{CDCl}_{3}\right)$ 


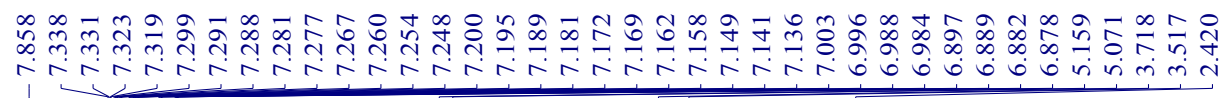
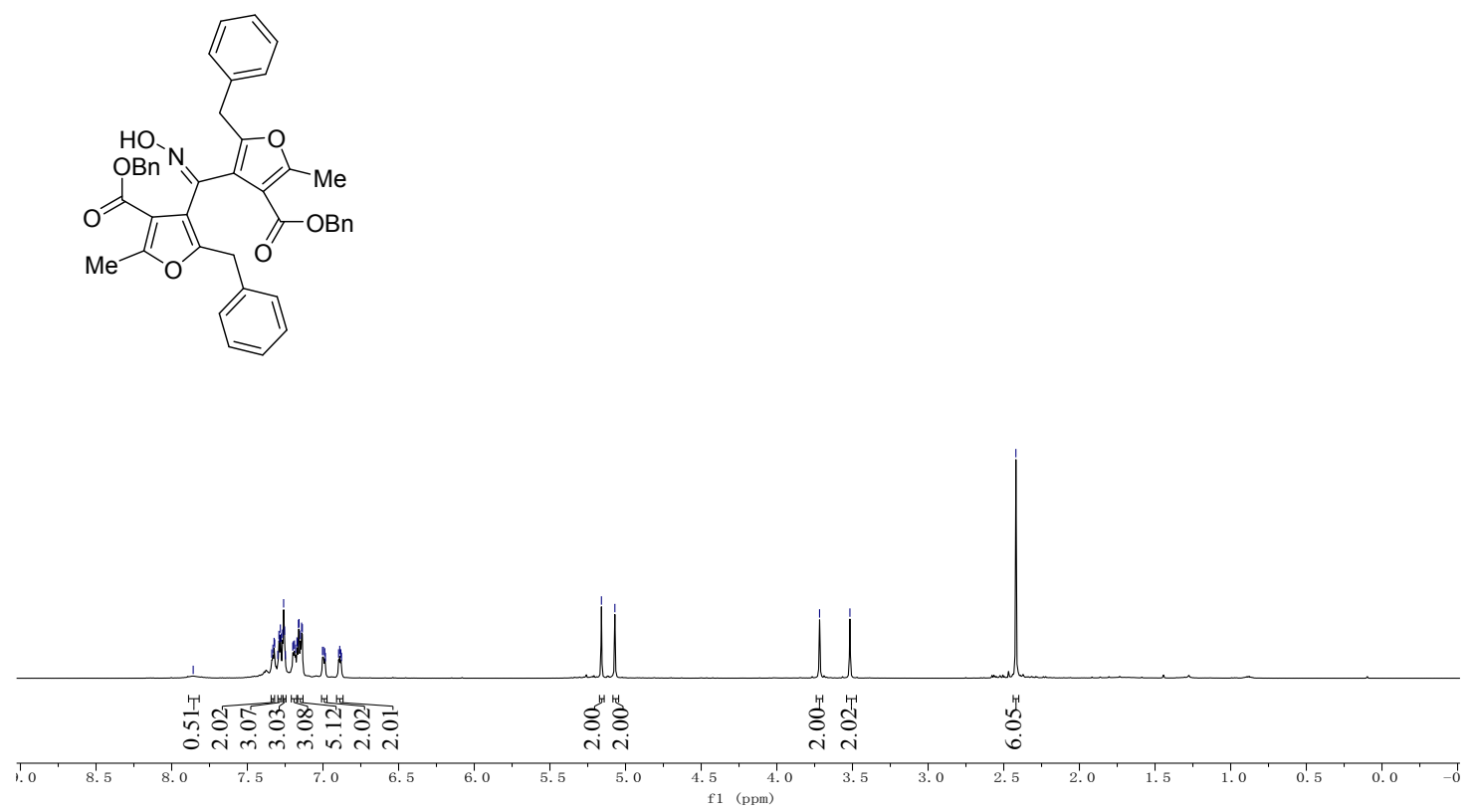

${ }^{1} \mathrm{H}$ NMR spectrum of $91\left(500 \mathrm{MHz}, \mathrm{CDCl}_{3}\right)$

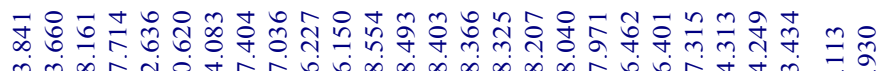

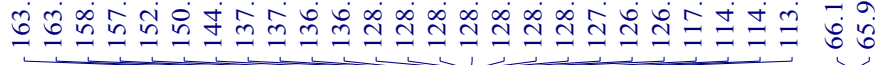

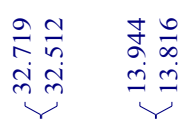<smiles>COc1oc(-c2ccccc2)c(Cc2ccccc2)c1C(=N)c1c(Cc2ccccc2)oc(C)c1C(=O)O</smiles>

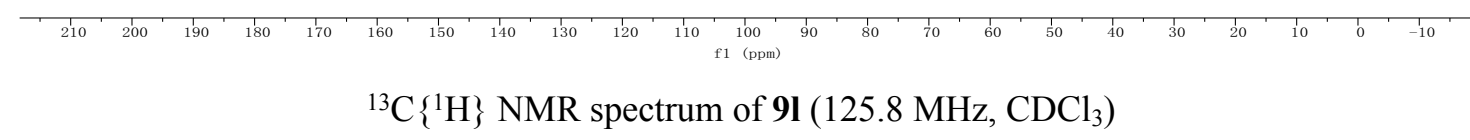


<smiles>CC(=O)c1cc(C(=O)c2ccccc2)oc1C</smiles>

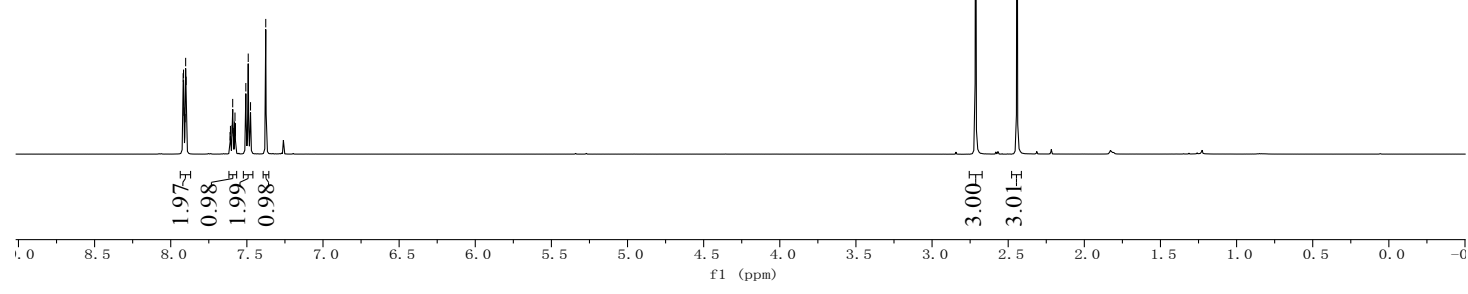

${ }^{1} \mathrm{H}$ NMR spectrum of $\mathbf{1 0 a}\left(500 \mathrm{MHz}, \mathrm{CDCl}_{3}\right)$
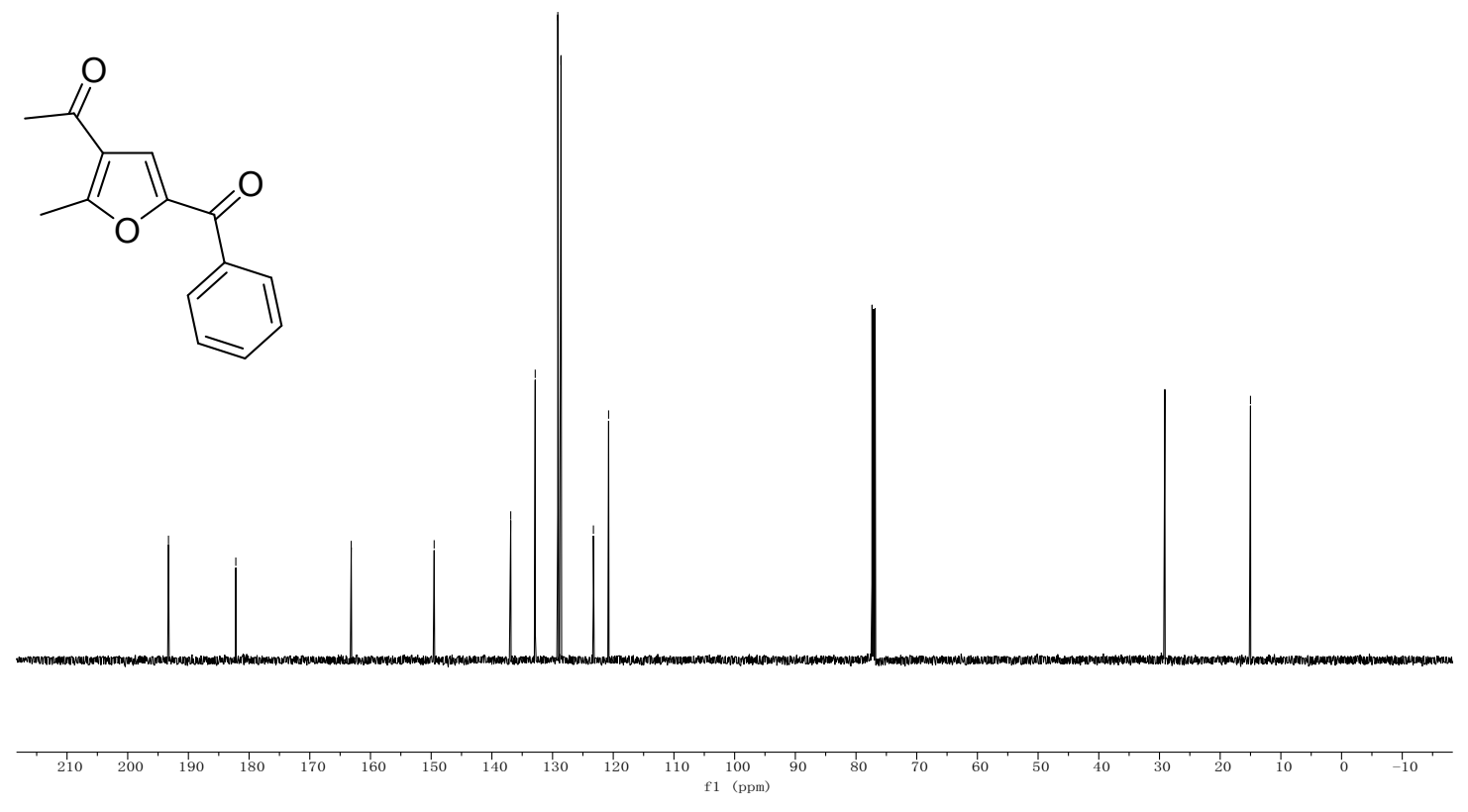

${ }^{13} \mathrm{C}\left\{{ }^{1} \mathrm{H}\right\}$ NMR spectrum of $\mathbf{1 0 a}\left(125.8 \mathrm{MHz}, \mathrm{CDCl}_{3}\right)$ 
<smiles>CC(=O)c1cc(C(=O)c2ccc(C)cc2)oc1C</smiles>

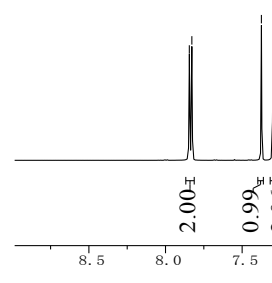

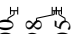

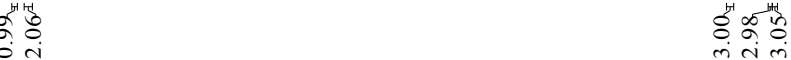

${ }^{1} \mathrm{H}$ NMR spectrum of $\mathbf{1 0 b}\left(500 \mathrm{MHz}, \mathrm{CDCl}_{3}\right)$

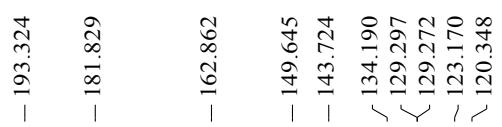
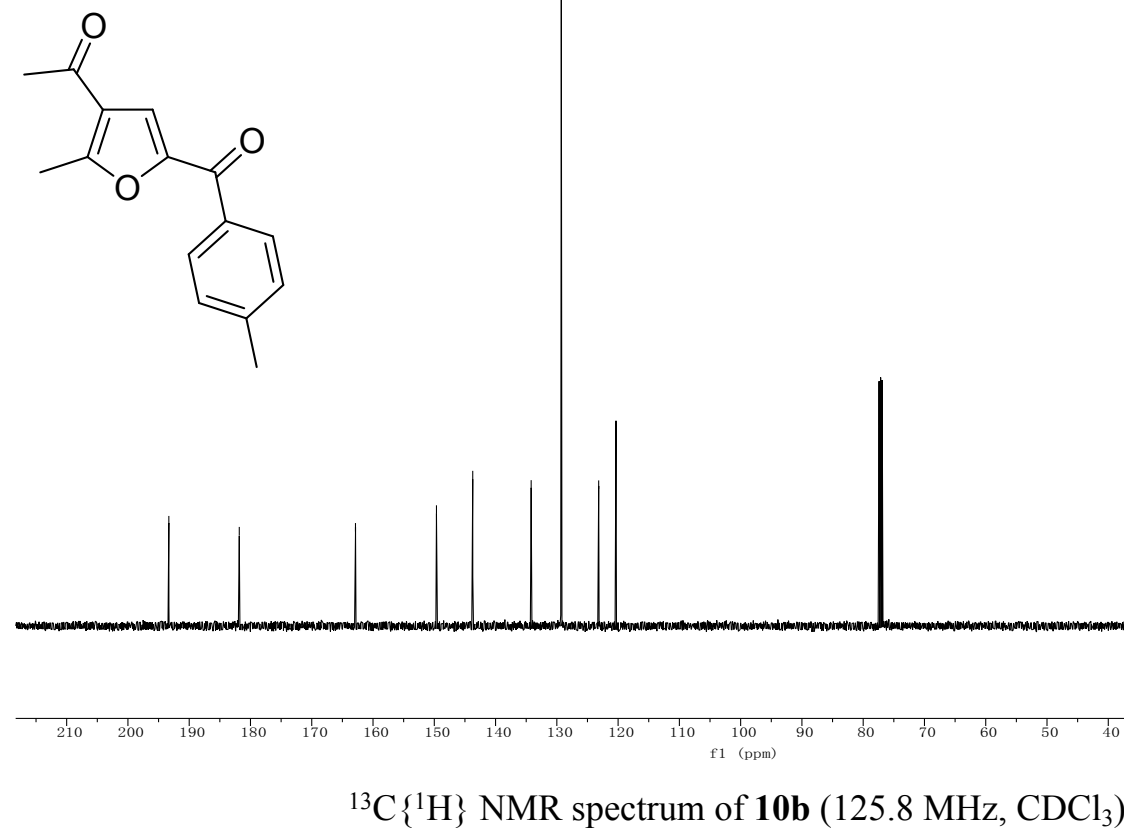


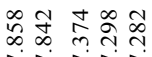

inis

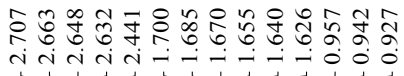

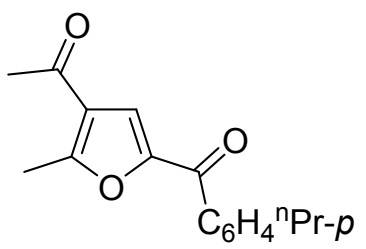

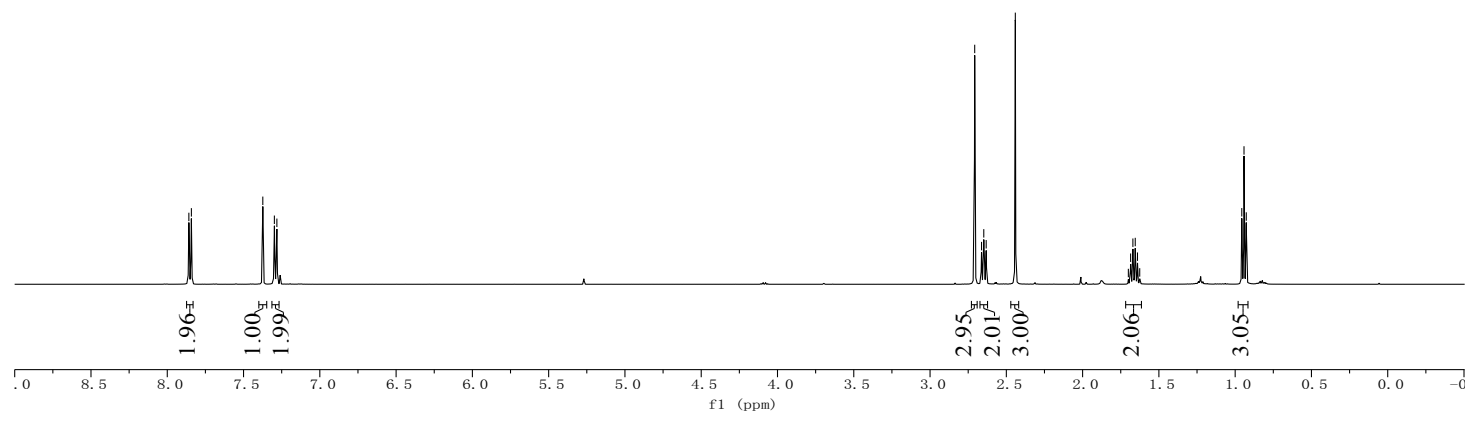

${ }^{1} \mathrm{H}$ NMR spectrum of $\mathbf{1 0 c}\left(500 \mathrm{MHz}, \mathrm{CDCl}_{3}\right)$

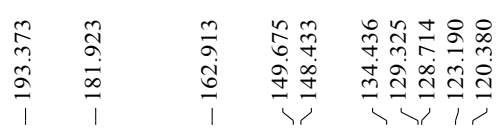
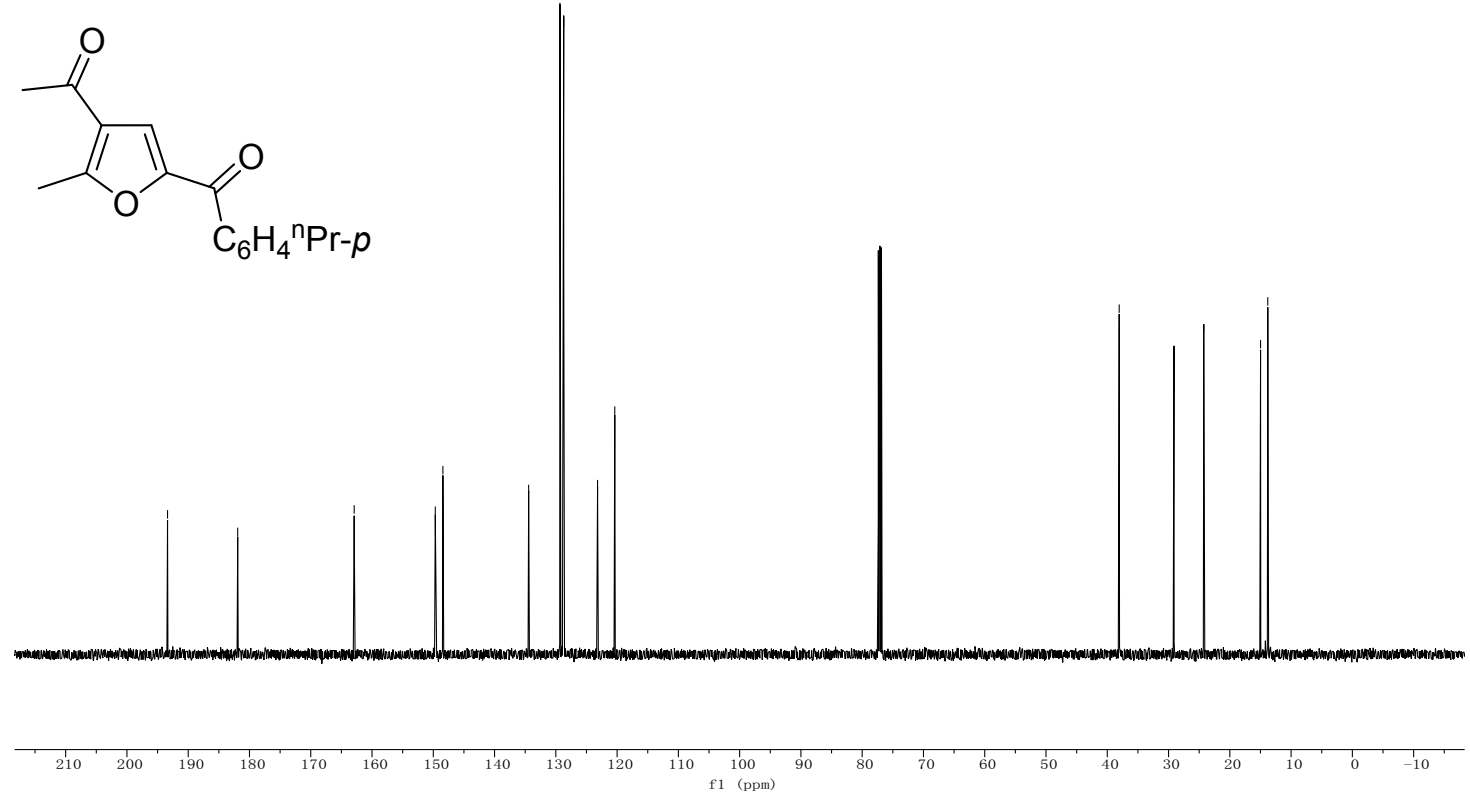

${ }^{13} \mathrm{C}\left\{{ }^{1} \mathrm{H}\right\}$ NMR spectrum of $\mathbf{1 0 c}\left(125.8 \mathrm{MHz}, \mathrm{CDCl}_{3}\right)$ 


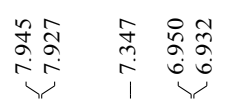

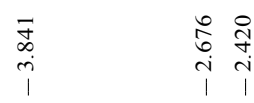

OMe
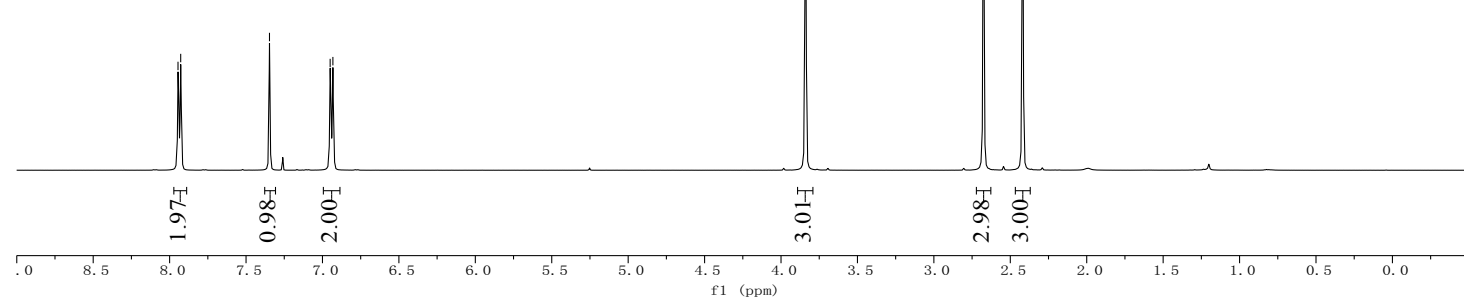

${ }^{1} \mathrm{H}$ NMR spectrum of $\mathbf{1 0 d}\left(500 \mathrm{MHz}, \mathrm{CDCl}_{3}\right)$

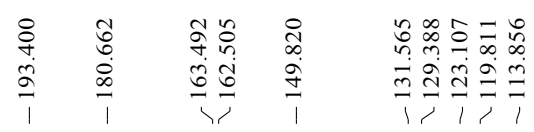

$\begin{array}{lll}0 & \infty & 0 \\ n & 0 & 0 \\ n & 0 & 0 \\ 0 & 1 & 1 \\ 0 & 0 & 1\end{array}$
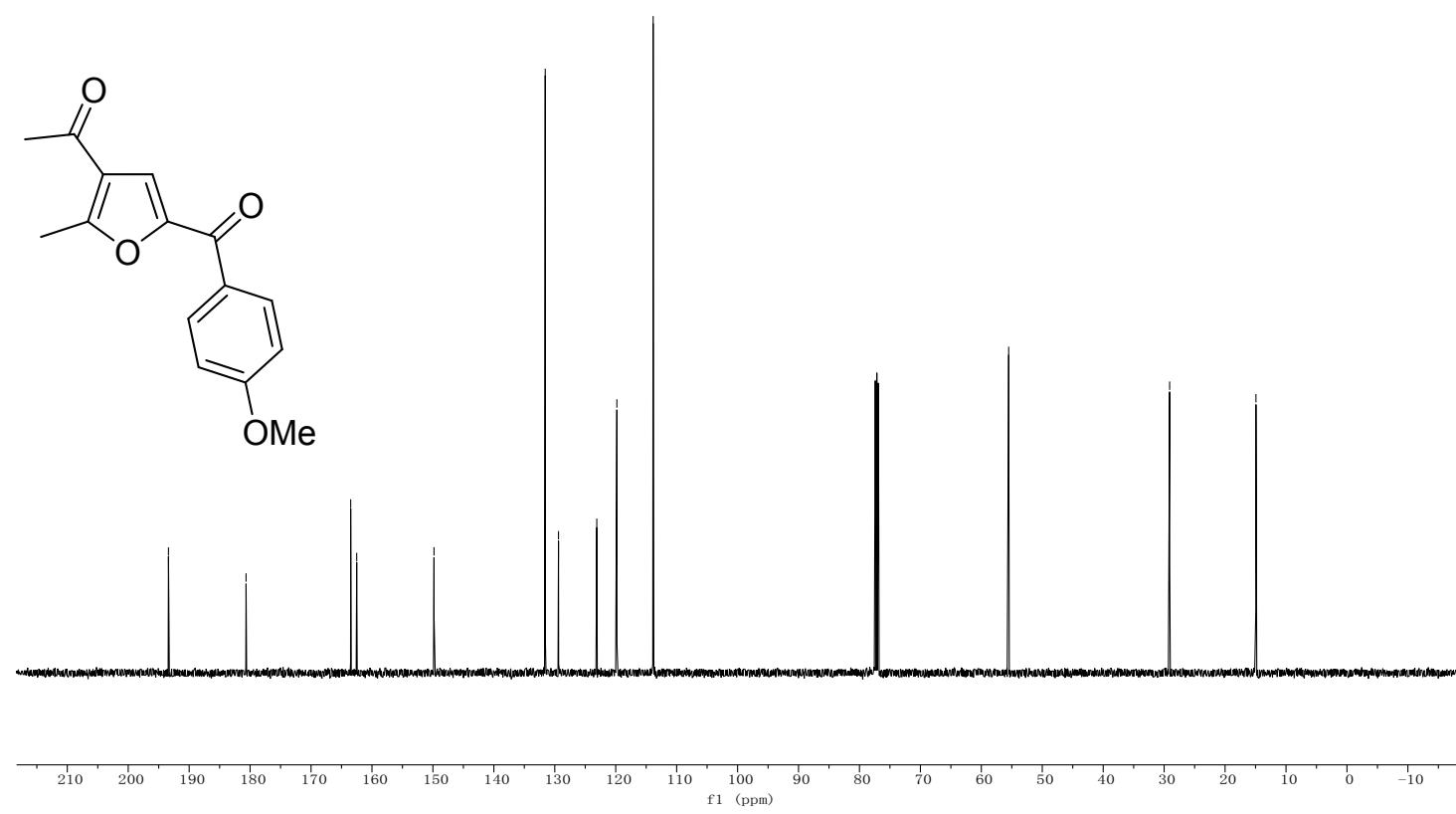

${ }^{13} \mathrm{C}\left\{{ }^{1} \mathrm{H}\right\}$ NMR spectrum of $\mathbf{1 0 d}\left(125.8 \mathrm{MHz}, \mathrm{CDCl}_{3}\right)$ 


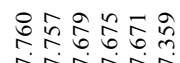

rivirion

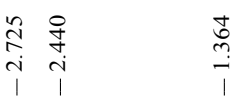<smiles>CC(=O)c1cc(C(=O)c2cc(C(C)(C)C)cc(C(C)(C)C)c2)oc1C</smiles>

$\mathrm{Bu}$

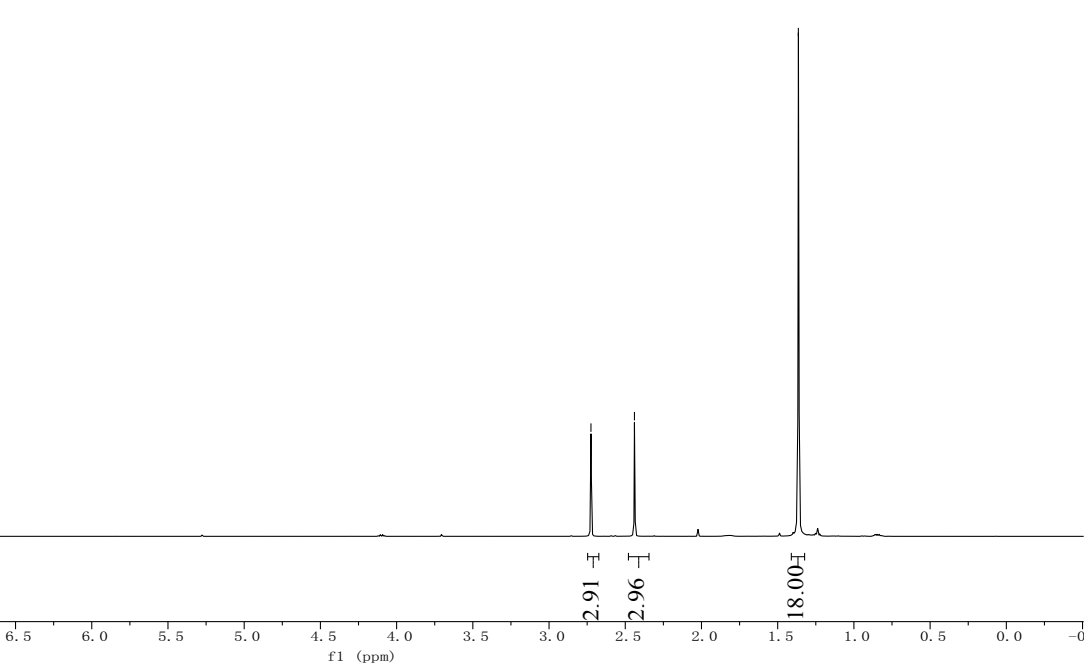

${ }^{1} \mathrm{H}$ NMR spectrum of $\mathbf{1 0 e}\left(500 \mathrm{MHz}, \mathrm{CDCl}_{3}\right)$

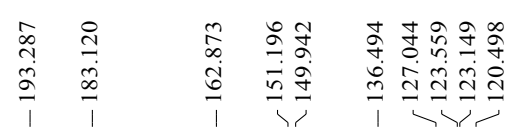

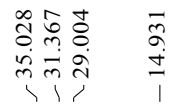<smiles>CC(=O)c1cc(C(=O)c2cc(C(C)(C)C)cc(C(C)(C)C)c2)oc1C</smiles>
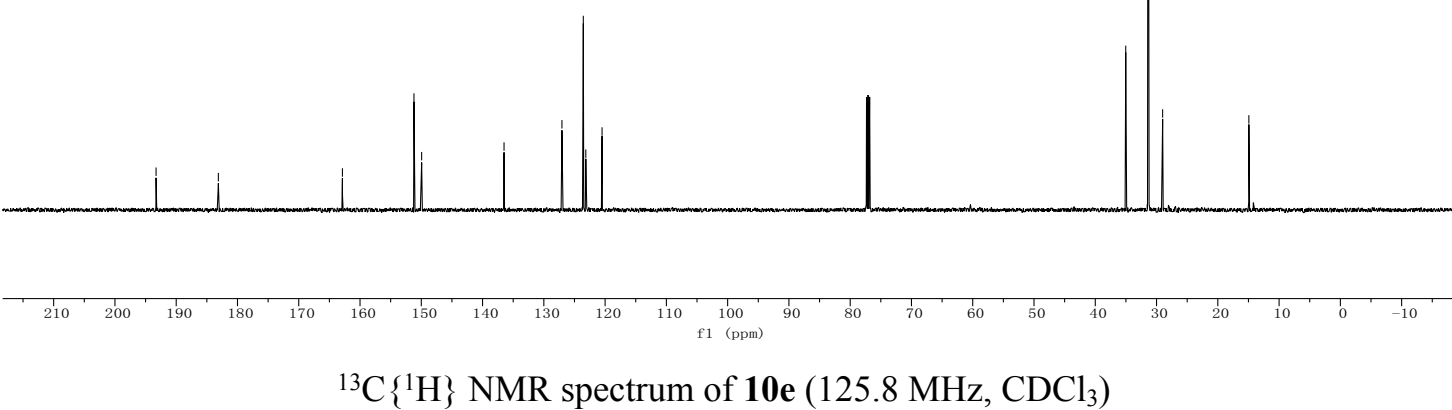


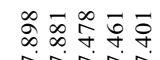

irin

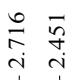

(l)

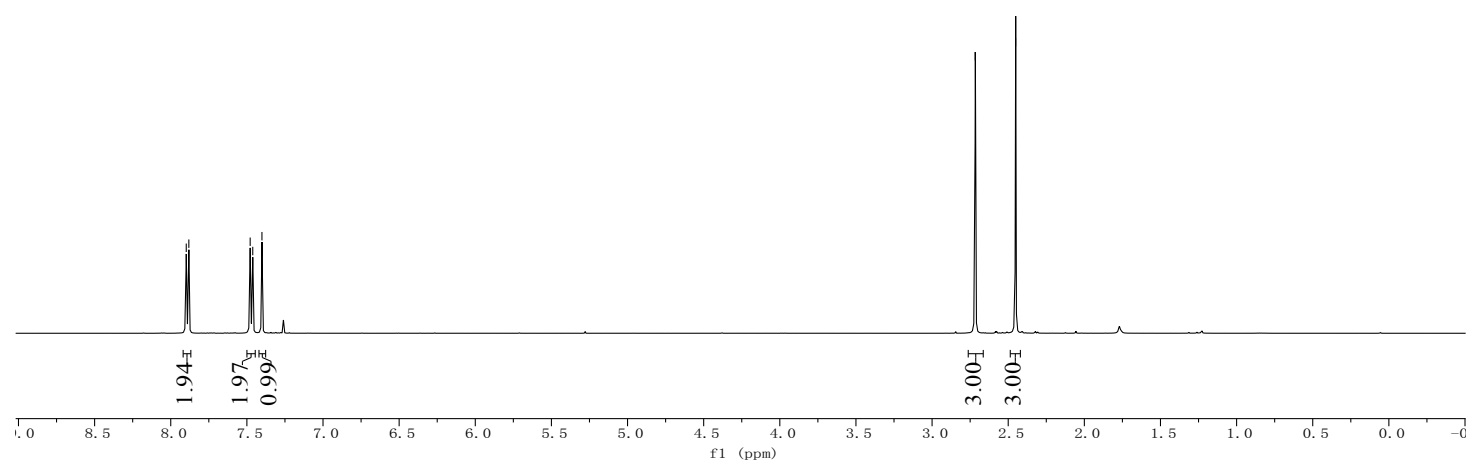

${ }^{1} \mathrm{H}$ NMR spectrum of $\mathbf{1 0 f}\left(500 \mathrm{MHz}, \mathrm{CDCl}_{3}\right)$

$$
\begin{array}{ccccc}
1 & 1 & 1 & 1 & 5 \\
0 & 0 & 1
\end{array}
$$<smiles>CC(=O)c1cc(C(=O)c2ccc(Cl)cc2)oc1C</smiles>

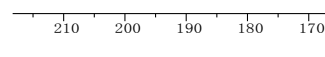

${ }^{13} \mathrm{C}\left\{{ }^{1} \mathrm{H}\right\}$ NMR spectrum of $\mathbf{1 0 f}\left(125.8 \mathrm{MHz}, \mathrm{CDCl}_{3}\right)$ 
(1)

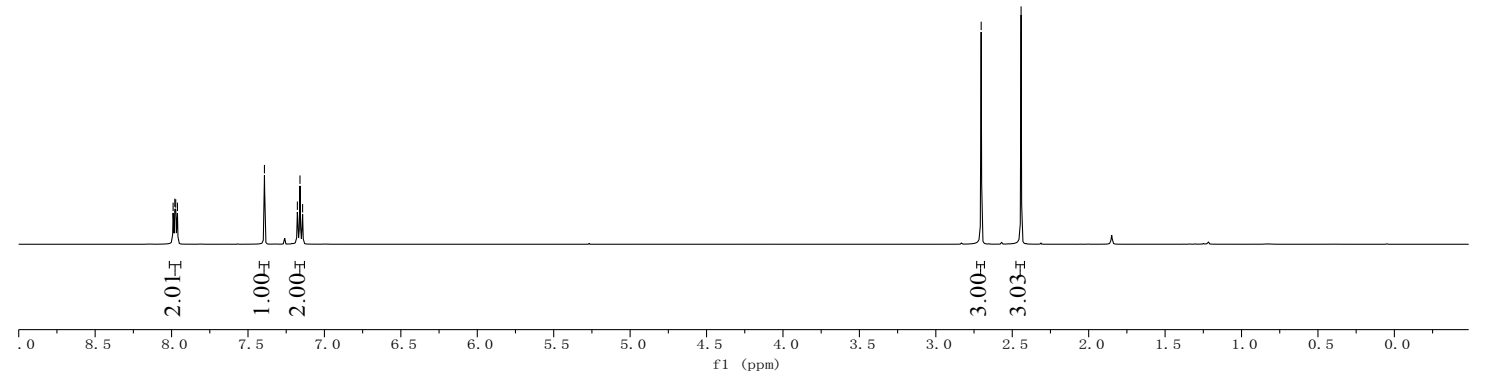

${ }^{1} \mathrm{H}$ NMR spectrum of $\mathbf{1 0 g}\left(500 \mathrm{MHz}, \mathrm{CDCl}_{3}\right)$

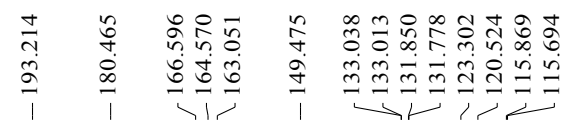<smiles>CC(=O)c1cc(C(=O)c2ccc(F)cc2)oc1C</smiles>

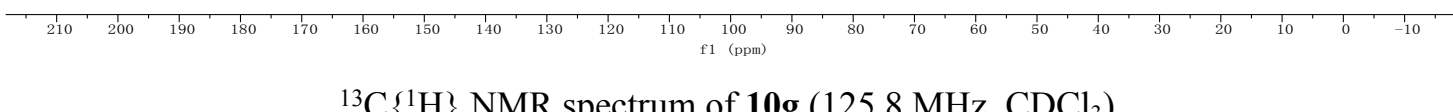

${ }^{13} \mathrm{C}\left\{{ }^{1} \mathrm{H}\right\}$ NMR spectrum of $\mathbf{1 0 g}\left(125.8 \mathrm{MHz}, \mathrm{CDCl}_{3}\right)$ 
<smiles>COC(=O)c1cc(C(=O)c2ccccc2)oc1C</smiles>

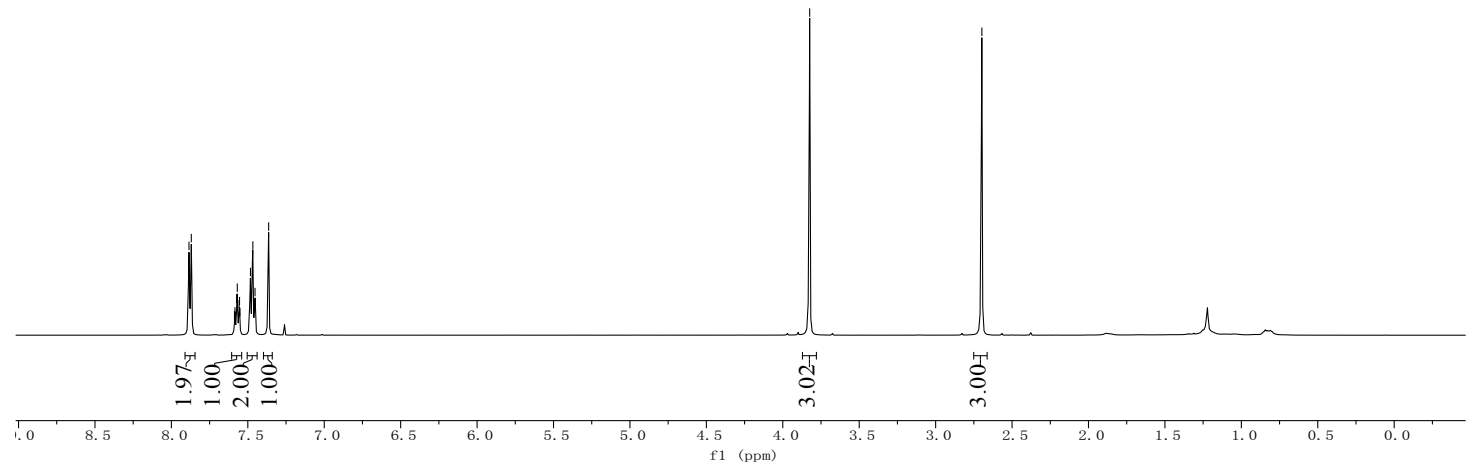

${ }^{1} \mathrm{H}$ NMR spectrum of $\mathbf{1 0 i}\left(500 \mathrm{MHz}, \mathrm{CDCl}_{3}\right)$

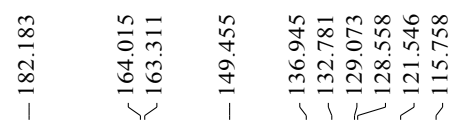

$\begin{array}{ll}\stackrel{0}{0} & \stackrel{6}{0} \\ \stackrel{0}{+} & \frac{1}{1}\end{array}$<smiles>COC(=O)c1cc(C(=O)c2ccccc2)oc1C</smiles>

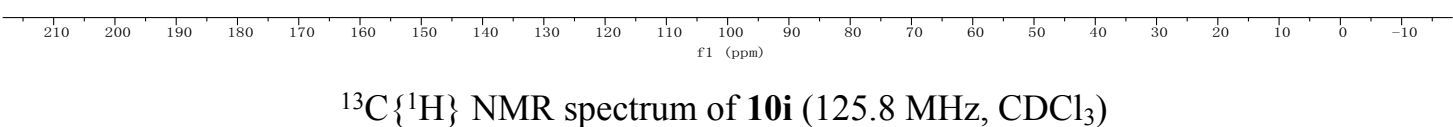

${ }^{13} \mathrm{C}\left\{{ }^{1} \mathrm{H}\right\}$ NMR spectrum of $\mathbf{1 0 i}\left(125.8 \mathrm{MHz}, \mathrm{CDCl}_{3}\right)$ 


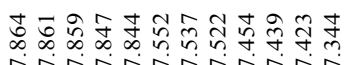

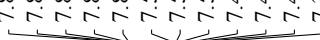

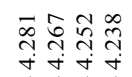

i.<smiles>CCOC(=O)c1cc(C(=O)c2ccccc2)oc1C</smiles>

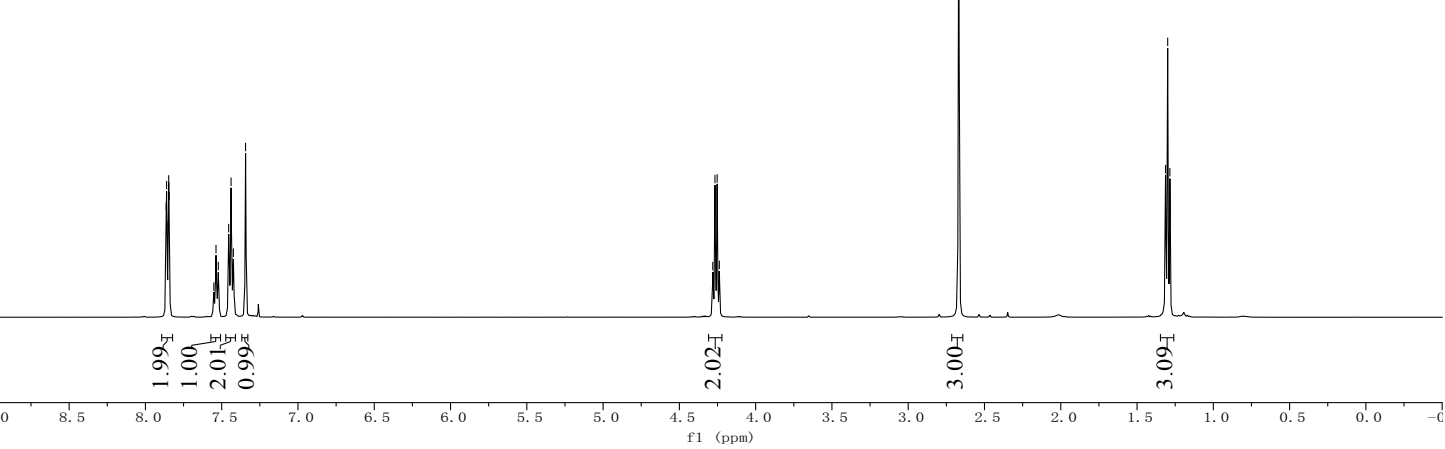

${ }^{1} \mathrm{H}$ NMR spectrum of $\mathbf{1 0 j}\left(500 \mathrm{MHz}, \mathrm{CDCl}_{3}\right)$

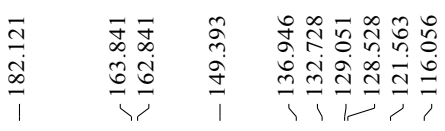

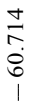

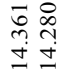<smiles>CCOC(=O)c1cc(C(=O)c2ccccc2)oc1C</smiles>
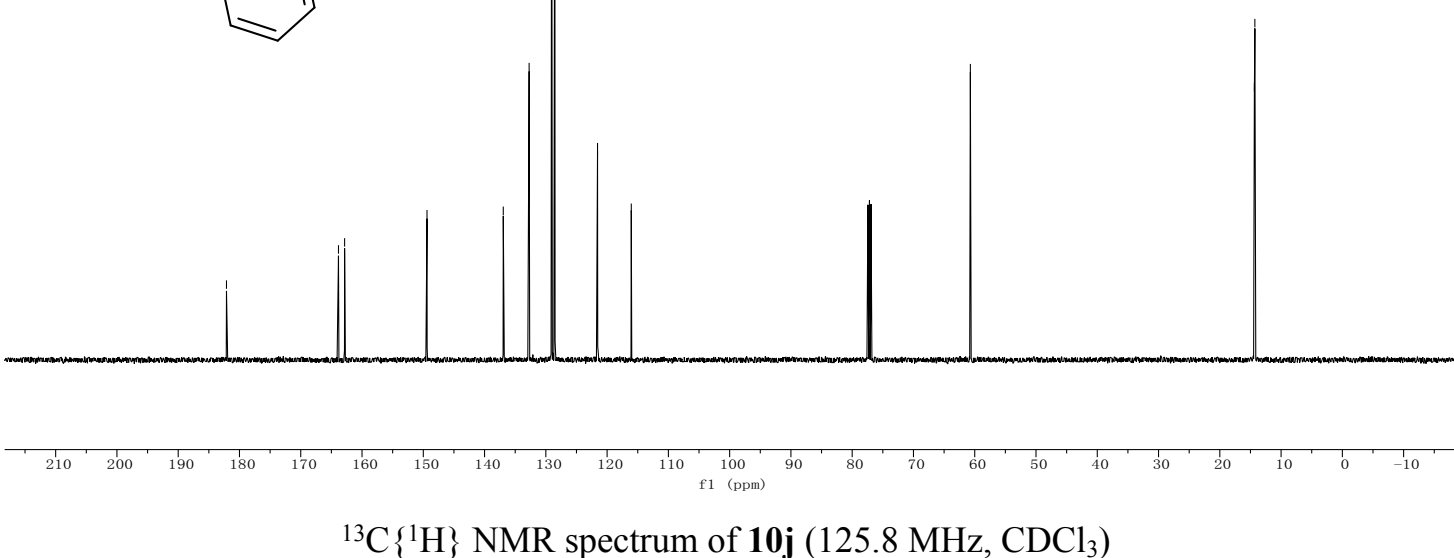

${ }^{13} \mathrm{C}\left\{{ }^{1} \mathrm{H}\right\}$ NMR spectrum of $\mathbf{1 0 j}\left(125.8 \mathrm{MHz}, \mathrm{CDCl}_{3}\right)$ 
<smiles>C=CCOC(=O)c1cc(C(=O)c2ccccc2)oc1C</smiles>

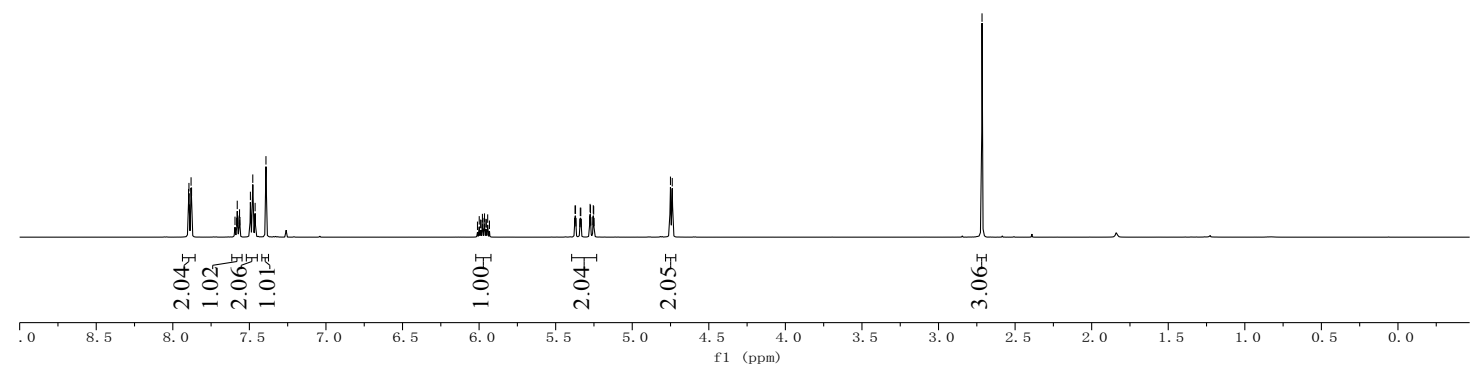

${ }^{1} \mathrm{H}$ NMR spectrum of $\mathbf{1 0 k}\left(500 \mathrm{MHz}, \mathrm{CDCl}_{3}\right)$
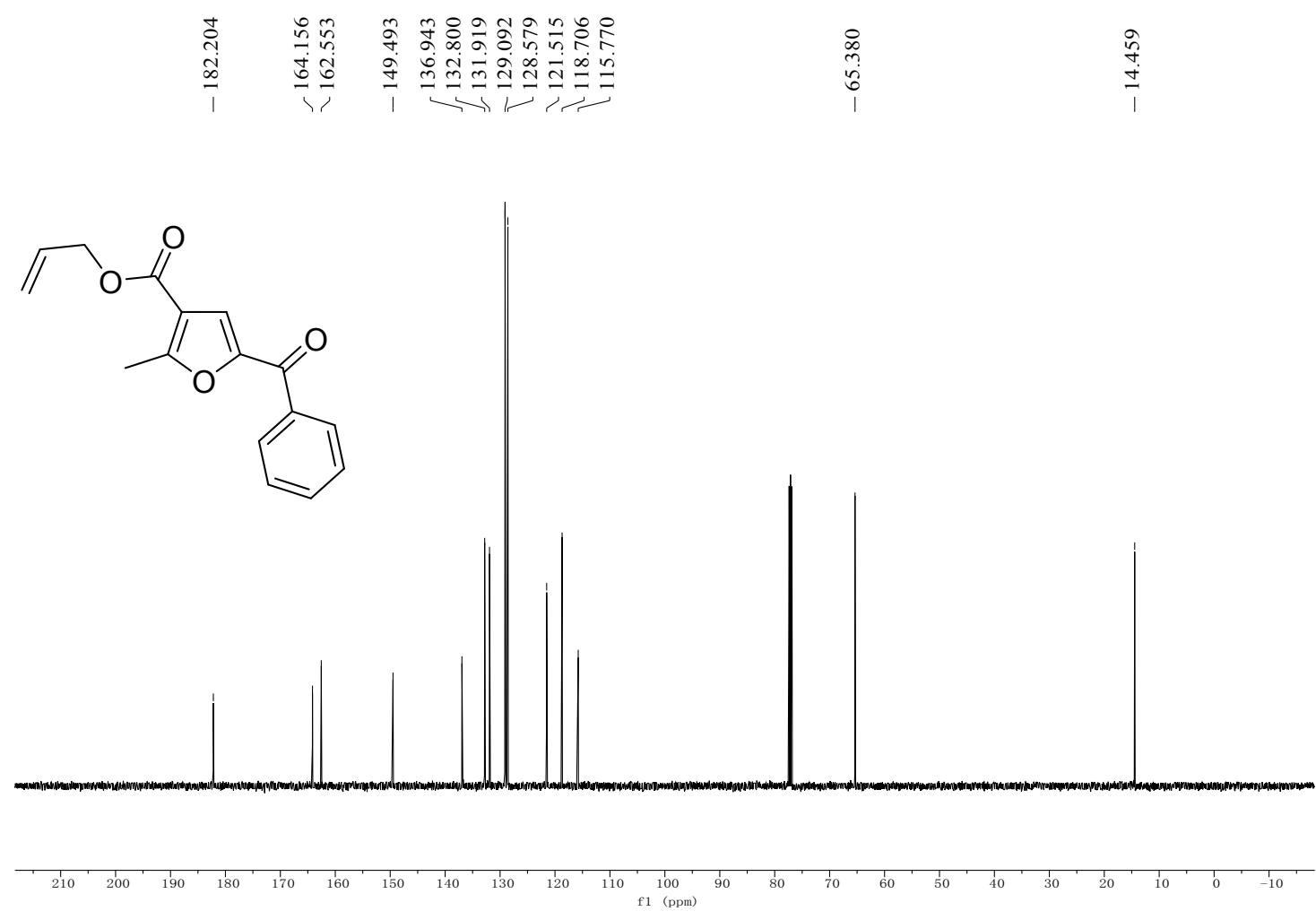

${ }^{13} \mathrm{C}\left\{{ }^{1} \mathrm{H}\right\}$ NMR spectrum of $\mathbf{1 0 k}\left(125.8 \mathrm{MHz}, \mathrm{CDCl}_{3}\right)$ 
<smiles>Cc1oc(C(=O)c2ccccc2)cc1C(=O)OCc1ccccc1</smiles>

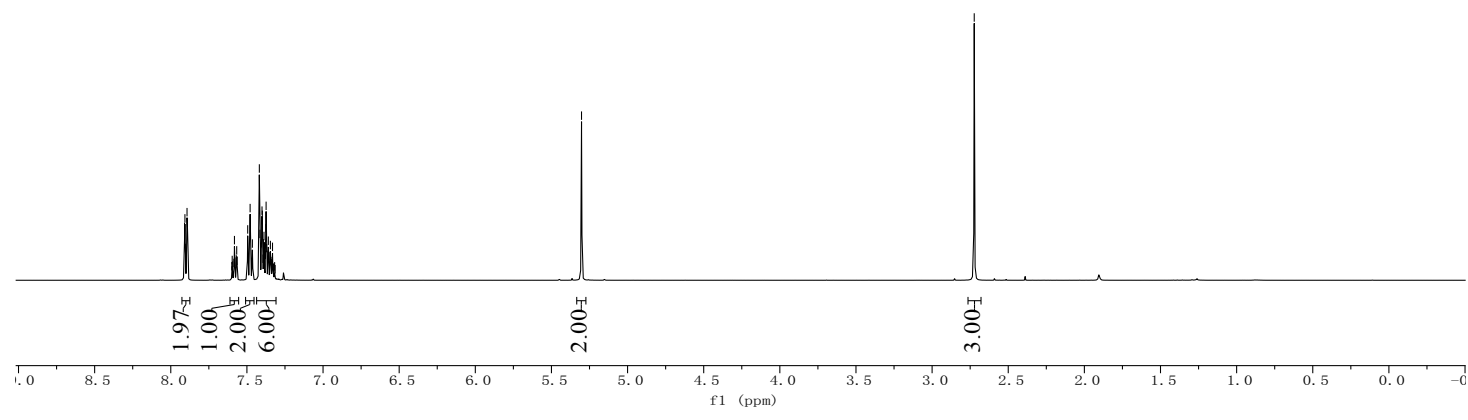

${ }^{1} \mathrm{H}$ NMR spectrum of $101\left(500 \mathrm{MHz}, \mathrm{CDCl}_{3}\right)$
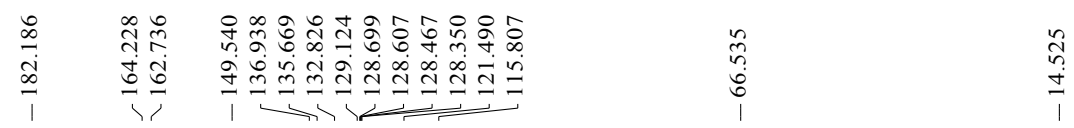<smiles>Cc1oc(C(=O)c2ccccc2)cc1C(=O)OCc1ccccc1</smiles>
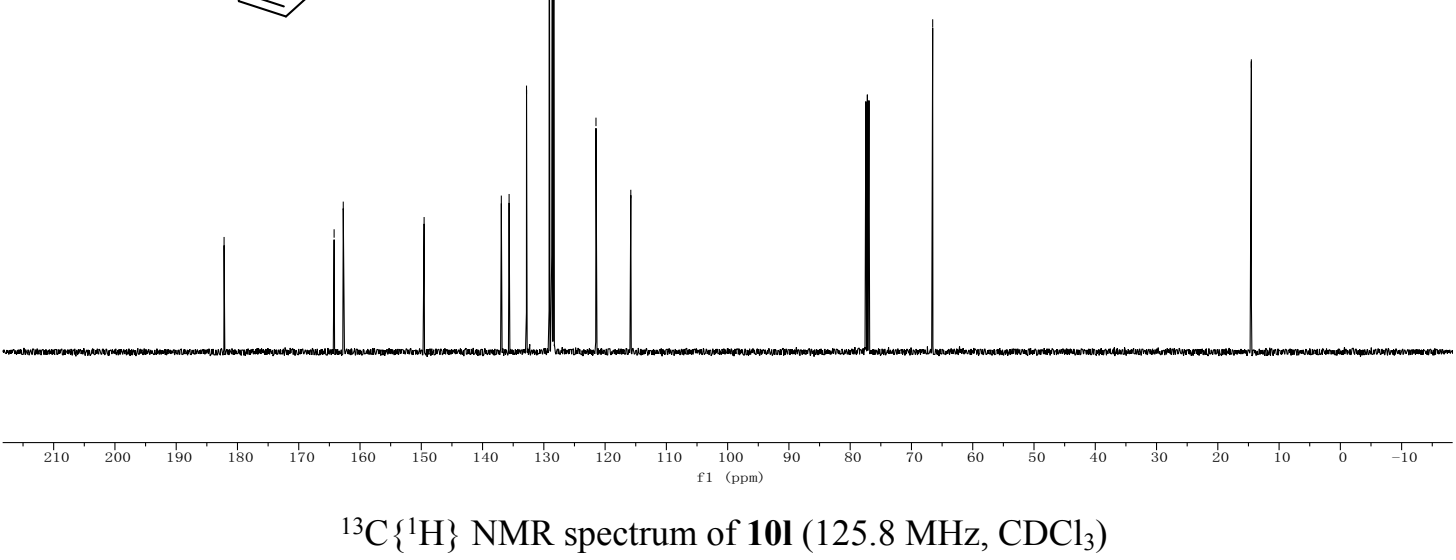
Ph

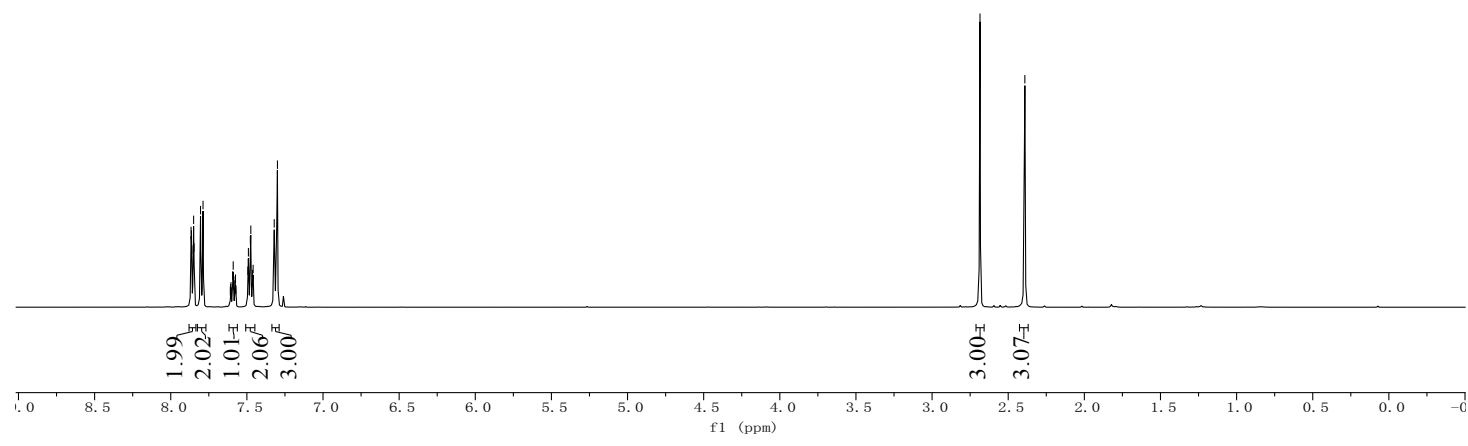

${ }^{1} \mathrm{H}$ NMR spectrum of $100\left(500 \mathrm{MHz}, \mathrm{CDCl}_{3}\right)$

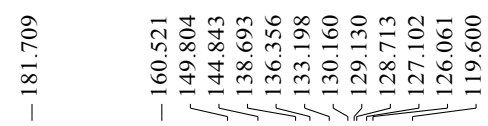<smiles>Cc1oc(C(=O)c2ccccc2)cc1F</smiles>

$\mathrm{Ph}$
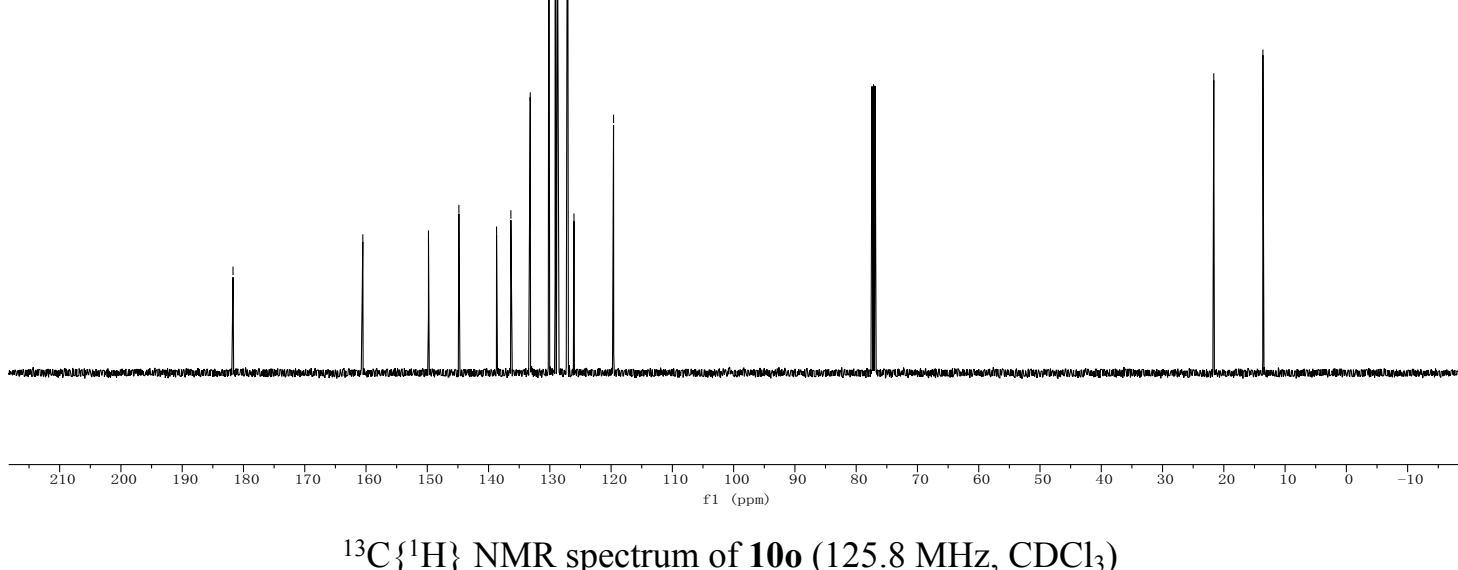

${ }^{13} \mathrm{C}\left\{{ }^{1} \mathrm{H}\right\}$ NMR spectrum of $\mathbf{1 0 o}\left(125.8 \mathrm{MHz}, \mathrm{CDCl}_{3}\right)$ 